\title{
Magnetic Properties of LSMO/STO Thin Films: Magnetocaloric, Spin Dynamics and Magnetic Viscosity Investigations
}

\author{
Navid Mottaghi \\ namottaghi@mix.wvu.edu
}

Follow this and additional works at: https://researchrepository.wvu.edu/etd

Part of the Instrumentation Commons, and the Other Physics Commons

\section{Recommended Citation}

Mottaghi, Navid, "Magnetic Properties of LSMO/STO Thin Films: Magnetocaloric, Spin Dynamics and Magnetic Viscosity Investigations" (2021). Graduate Theses, Dissertations, and Problem Reports. 8306. https://researchrepository.wvu.edu/etd/8306

This Dissertation is protected by copyright and/or related rights. It has been brought to you by the The Research Repository @ WVU with permission from the rights-holder(s). You are free to use this Dissertation in any way that is permitted by the copyright and related rights legislation that applies to your use. For other uses you must obtain permission from the rights-holder(s) directly, unless additional rights are indicated by a Creative Commons license in the record and/ or on the work itself. This Dissertation has been accepted for inclusion in WVU Graduate Theses, Dissertations, and Problem Reports collection by an authorized administrator of The Research Repository @ WVU.

For more information, please contact researchrepository@mail.wvu.edu. 


\title{
Magnetic Properties of LSMO/STO Thin Films: Magnetocaloric, Spin Dynamics and Magnetic Viscosity Investigations
}

\author{
Navid Mottaghi \\ Dissertation submitted to the \\ Eberly College of Arts and Sciences \\ at West Virginia University \\ In partial fulfillment of the requirements for the degree of \\ Doctor of Philosophy \\ in \\ Physics \\ Holcomb, Mikel B, Ph.D., Chair \\ Abdul-Razzaq, Wathiq, Ph.D. \\ Stanescu, Tudor D, Ph.D. \\ Johnson, Matthew B, Ph.D. \\ Song, Xueyan, Ph.D. \\ Department of Physics and Astronomy \\ Morgantown, West Virginia \\ 2021
}

Keywords: Thin films, Magnetic Inhomogeneity, Negative magnetization, Hysteresis loop inversion, Magnetocaloric Studies, Relative Cooling Power, Negative Magnetic Relaxation, Spin Dynamics, Vogel-Fulcher Law, Cole-Cole analysis

Copyright 2021 Navid Mottaghi 


\begin{abstract}
Magnetic Properties of LSMO/STO Thin Films: Magnetocaloric, Spin Dynamics and Magnetic

Viscosity Investigations
\end{abstract}

\title{
Navid Mottaghi
}

While other films are discussed, this dissertation will focus on detailed studies of the dc and ac bulk magnetometry in a characteristic $7.6 \mathrm{~nm}$ thin film of $\mathrm{La}_{0.7} \mathrm{Sr}_{0.3} \mathrm{MnO}_{3}$ grown on $\mathrm{SrTiO}_{3}(001)$. The de bulk magnetometry measurements show that the sample is magnetically inhomogeneous. Temperature variation of magnetization ( $M$ vs. $T$ ) was measured in zero-field-cooled and field-cooled protocols to determine the blocking temperature $T_{\mathrm{B}}$ in different applied magnetic fields. The field variation of $T_{\mathrm{B}}$ is interpreted as the presence of embedded spin clusters of $1.4 \mathrm{~nm}$. Moreover, the $M$ vs. $T$ measurements show the presence of negative magnetization in low applied fields of $H=50$ Oe and 100 Oe.

The field variation of magnetization was also measured by performing hysteresis loop (HL) measurements. These measurements were performed from $5 \mathrm{~K}$ to $400 \mathrm{~K}$ and the HL parameters are calculated to detect the magnetic state of the sample in this temperature region. These measurements show that this sample has superparamagnetic spin clusters with $T_{\mathrm{B}}=240 \mathrm{~K}$ and a ferromagnetic state with an ordering temperature $T_{\mathrm{C}}=290 \mathrm{~K}$. Within the temperature region of $T_{\mathrm{B}} \leq T \leq T_{\mathrm{C}}$, the $\mathrm{HL}$ is inverted whereas negative remanence magnetization (NRM) appears in the mixed SPM and FM phases leading to the antialignment spin of both magnetic phases with respect to each other.

The presence of SPM and FM phases produces energy barriers that create different magnetic states. Therefore, to under the predominant magnetization processes in this magnetically inhomogeneous sample, the magnetic viscosity measurements were performed. Magnetic viscosity $S$ measurements were performed by cooling the sample $H=50$ Oe to the measured temperature and magnetization was measured as a function of time in $H=0$. Magnetization has logarithmic decay which from the fit to the magnetization: $M$ $(t)=M(0)-S \ln (t)$, with time $t$ up to $2 \mathrm{~h}$, shows a peak at $230 \mathrm{~K}$ above which $M(2 \mathrm{~h})$ switches to negative values for temperatures up to the $T_{\mathrm{C}}$ of the sample. Here it is argued that this negative magnetization results from a magnetic interaction between the SPM and FM phases.

In an effort to explore applications of this magnetic phenomenon, magnetocaloric studies are reported here to study the thermodynamic properties of the magnetic phases. This study proves the sample is magnetically inhomogeneous since the magnetic entropy change $\left(-\Delta S_{M}\right) v s$. temperature data has two broad peaks one close to the $T_{\mathrm{B}}$ and the other centered near $T \sim T_{\mathrm{C}}$. Detailed analysis of $-\Delta S_{M}(T, H) v s . T$ data is used to determine the volume fraction of the magnetic phases which is in good agreement with the dead layer calculations by studying saturation magnetization of the sample. The relative cooling power of this film is less than that of nanoparticles and the reason why is discussed.

To understand the strength of interaction between the magnetic phases and spin dynamics of the sample, the ac magnetic susceptibilities $\chi^{\prime}$ and $\chi^{\prime \prime}$ were performed. AC magnetic susceptibilities $\chi^{\prime}$ and $\chi^{\prime \prime}$ were carried out to study the nature of magnetic phase transitions at $T_{\mathrm{B}}$ and the $T_{\mathrm{C}}$ of the sample in detail. The temperature dependence of $\chi^{\prime}$ and $\chi^{\prime \prime}$ measured at ac frequencies in the range of $0.1-10 \mathrm{kHz}$ shows a broad peak at $T_{\mathrm{B}}$ associated with the SPM phase present in the $1.4 \mathrm{~nm}$ surface layer and the frequencydependent peak near 270 associated with $T_{\mathrm{C}}$. The Cole-Cole analysis of temperature dependence of $\chi^{\prime}$ and $\chi^{\prime \prime}$ is used to calculate the mean relaxation time which is shown to fit the 
Vogel-Fulcher law: $\tau=\tau_{0} \exp \left[\Delta E / k_{\mathrm{B}}\left(T-T_{0}\right)\right]$ with $T_{0}=245 \mathrm{~K}$ close to the $T_{\mathrm{B}}$ and $\Delta E / k_{\mathrm{B}}=270 \mathrm{~K}=T_{\mathrm{C}}$, and $\tau_{0}=1.2 \times 10^{-9} \mathrm{~s}$. The reported results in this dissertation shed light on the origin of magnetic dead layers and their magnetic properties that can provide an opportunity for new types of magnetic devices. 


\section{Acknowledgments}

I would like to express my thanks to all the people who could help and support me while I was working on my Ph.D. at West Virginia University.

I would like to thank my Ph.D. advisor, Dr. Mikel. B. Holcomb for her support, encouragement, and kindness to pursue my Ph.D. degree. I would like to thank Dr. Mohindar S. Seehra for his excellent guidance through my work and also for his endless effort to guide me through magnetic measurements and analysis.

The work in this dissertation was the combination of the help from Dr. Hoclomb's group members in growing the samples, structural and magnetic characterizations.

Thanks to my collaborator: Dr. Menka Jain and Jiahang Shi from the University of Connecticut and Dr. Ryan Baumbach from the National High Magnetic Field Laboratory who helped me to perform the ac magnetic susceptibility measurements.

Thanks to my committee members Dr. Abdul-Razzaq, Wathiq, Dr. Tudor Stanescu, Dr. Matthew Jonson, and Dr. Xueyan Song for their time and efforts, and encouragement to work harder in my Ph.D. work.

Additionally, I like to thank, Harley Hart and Dr. Qiang Wang, from Shared Research Facilities for their effort in maintaining the instruments and mentoring me in running the fabrication and characterization machines.

The funding support was a necessity in this work and I would like to thank National Science Foundation (NSF) (No. DMR-1608656) for growth and optimization and the U.S. Department of Energy (DOE) (No. DE-SC0016176)). This work has been included in our sample database made possible by NASA WV EPSCoR Grant No. NNX15AK74A.

Finally, I would like to thank my family for their guidance, patience, encouragement, and their emotional support. 


\section{Table of Contents}

Chapter 1 Background and motivation.................................................................................... 1

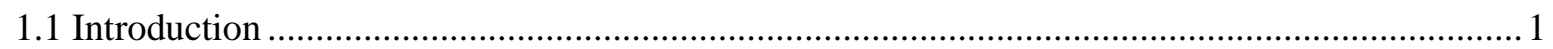

1.2 A Brief Discussion on the Basics of Magnetism and Magnetic Phases ...................................... 1

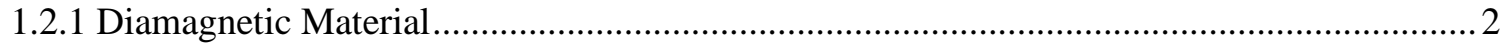

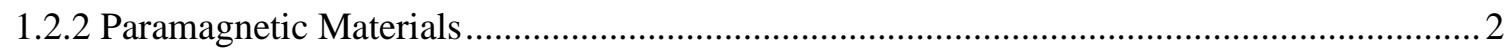

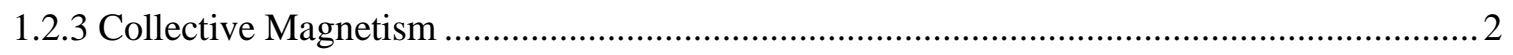

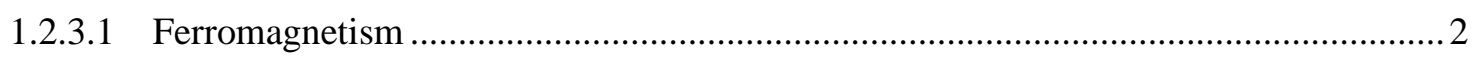

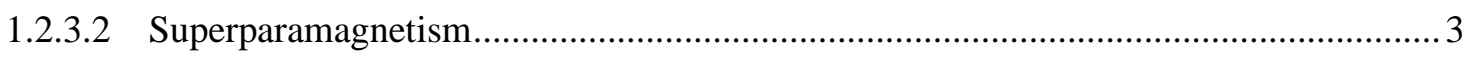

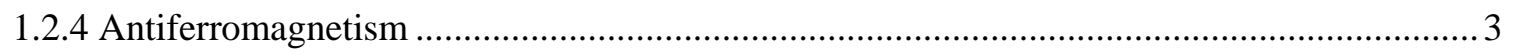

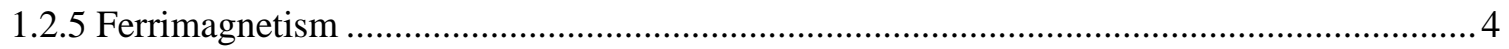

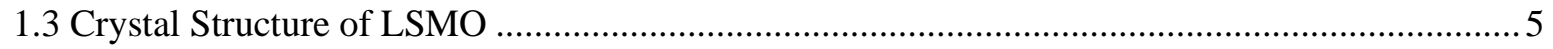

1.3.1 Magnetic Behavior in Manganites ........................................................................... 6

1.4 Studies on LSMO Nanoparticles and Thin Films to Understand the Dead Layers ...................... 7

1.5 Motivation for this Research and Outline of Approaches .................................................... 10

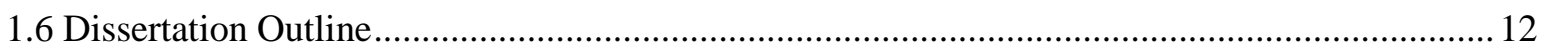

Chapter 2 Thin Film Fabrication Process, Structural and Magnetic Characterization of LSMO Films13

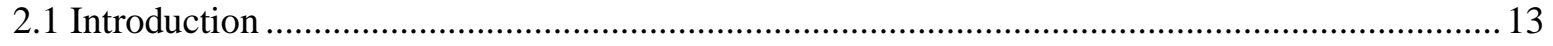

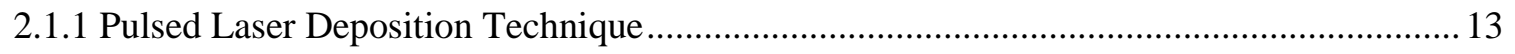

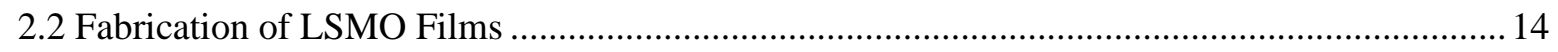

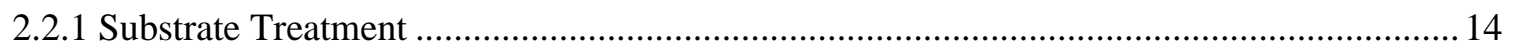

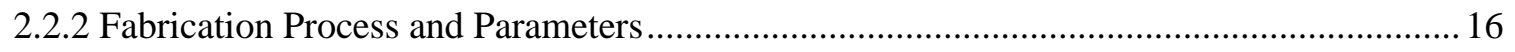

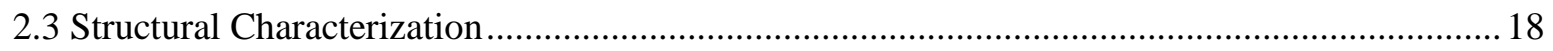

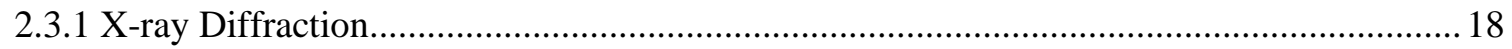

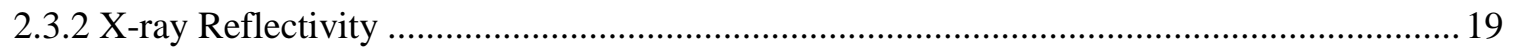

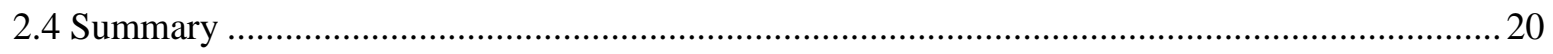


Chapter 3 Investigation of the Magnetic Dead Layer Properties by DC- Magnetometry Measurements 21

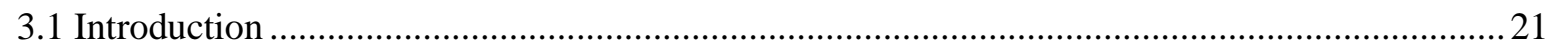

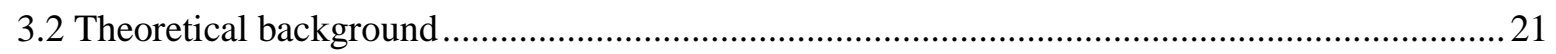

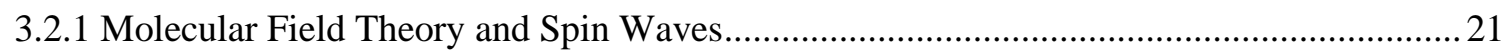

3.3 What is the Relation Between the Molecular Field and the Exchange Interaction?..................22

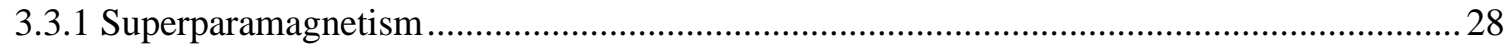

3.3.1.1 Blocking Temperature, Energy Barrier, and Magnetic Viscosity..............................28

3.4 Experimental Results and Discussions: DC Magnetometry Measurements and Modeling .........32

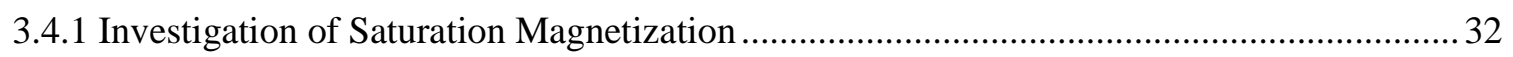

3.4.2 Temperature Variation of Magnetization and Blocking Temperature …............................. 34

3.4.3 Temperature Dependence of Hysteresis Loops and Loop Parameters ................................ 37

3.5 DC Magnetic Relaxation Measurements: Magnetic Viscosity Investigation............................ 43

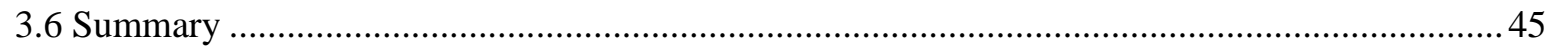

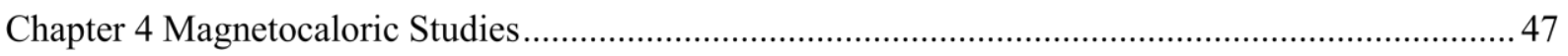

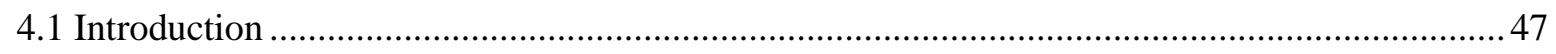

4.2 Magnetic Field and Temperature Dependence of The Change of Magnetic Entropy ................ 47

4.3 Magnetic Phase Volume Fraction Calculation From $\Delta \mathrm{S}_{\mathrm{M}}(\mathrm{T}, \mathrm{H})$ vs. $T$ Graphs at Different $H$.... 50

4.4 Magnetic Field Dependence of the Change of Magnetic Entropy ...........................................51

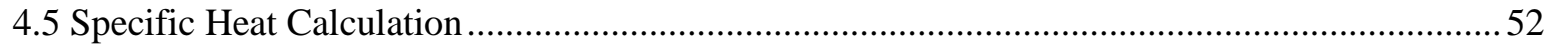

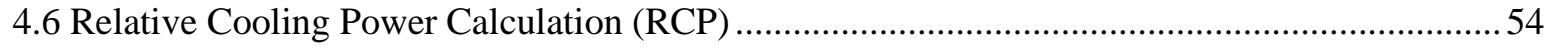

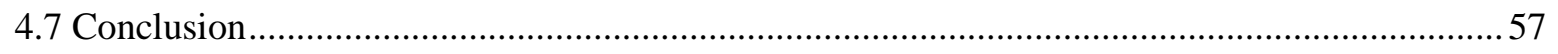

Chapter 5 Temperature and Frequency Dependence of AC Susceptibility ........................................5 59

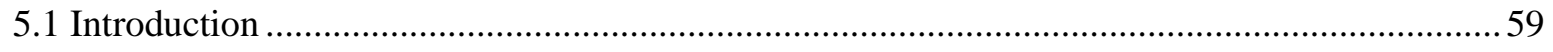

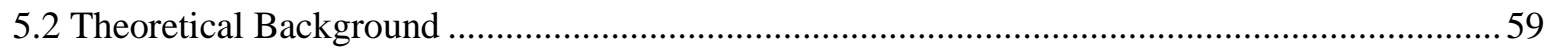

5.2.1 Temperature and Frequency-Dependent on AC susceptibilities ........................................59

5.3 Investigation of Temperature and Frequency Dependence of AC Susceptibilities .................... 61 


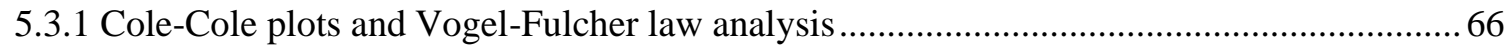

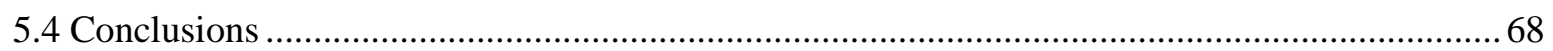

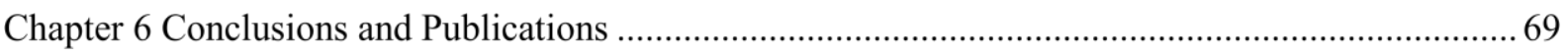

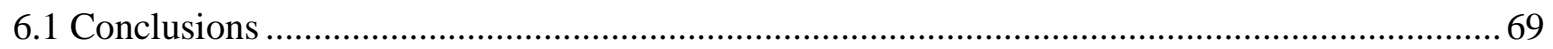

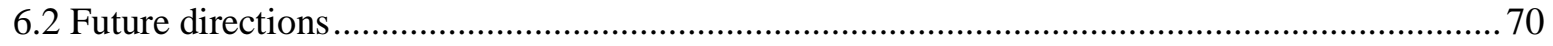

6.3 Publications and Presentations of the Author Related to this Dissertation ............................... 71

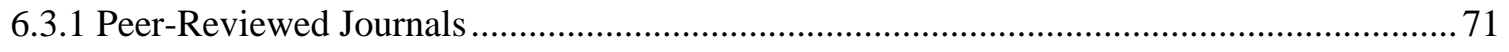

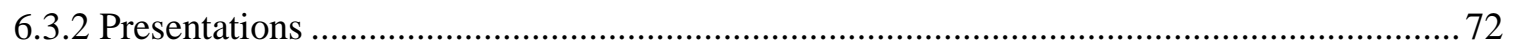




\section{List of Figures}

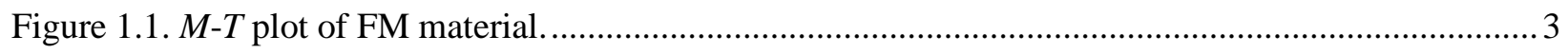

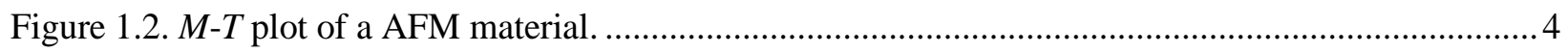

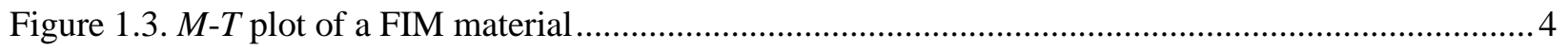

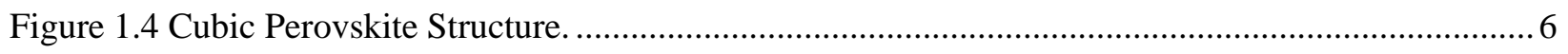

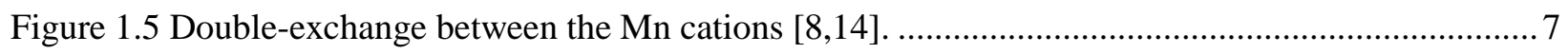

Figure 1.6 HRTEM of (a) LCMO NT and (b) LSMO NP. The surface layer with the thickness of $2 \mathrm{~nm}$ is indicated by a dotted line. (c) $M-T$ measurements of LCMO NTs and LSMO NPs in ZFC and FC cycles measured at $H=12 \mathrm{kOe}$. Reproduced from, [22] with the permission of AIP Publishing......................... 8 Figure 1.7 M-T plots of LSMO films (a) grown at different oxygen pressures, (b) with different thicknesses. Reproduced from [3] with the permission of APS Publishing.......................................................... 9 Figure 1.8. Variation of the $M_{\mathrm{S}}$ and $T_{\mathrm{C}}$ with thickness $D$ of the LSMO films. Different symbols represent data from different groups as follows: solid blue rectangle [3], solid red circle [27], open green diamond [23]. The solid lines are fit to the equation-(1-5) in (a) and equation-(1-6) in (b) with the listed parameters.

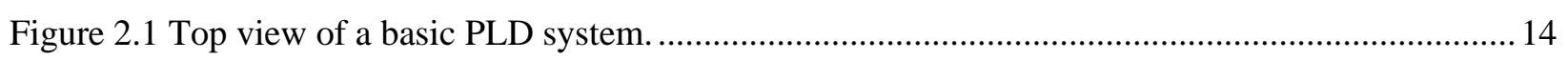

Figure 2.2 (a) cubic structure of $\mathrm{SrTiO}_{3}$, (b) $\mathrm{TiO}_{2}$ plane, (c) $\mathrm{SrO}$ plane ................................................ 15

Figure 2.3. (a) AFM image of height, (b) line profile which is shown by a black solid line.................... 16

Figure 2.4 RHEED diffraction condition with the Ewald's sphere, Laue circle and the reciprocal rod

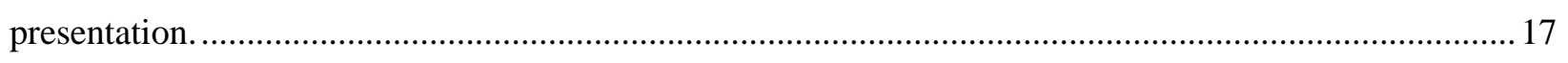

Figure 2.5. RHEED diffraction patterns of (a) STO substrate (b) $7.6 \mathrm{~nm}$ LSMO/STO after growth........ 18

Figure 2.6. XRD scan of LSMO films (a) $7.6 \mathrm{~nm}$ LSMO/STO and (b) $32 \mathrm{~nm} \mathrm{LSMO/STO} \mathrm{film..............} 19$

Figure 2.7. XRR profile for the $7.6 \mathrm{~nm} \mathrm{LSMO/STO}$ thin film sample. The blue dashed line and the continuous red line are the fits to one-layer and the two-layer models respectively...............................20

Figure 3.1. The ZFC hysteresis loop was measured at $5 \mathrm{~K}$ before and after background subtraction........ 33

Figure 3.2. Temperature variation of $M_{\mathrm{S}}$ taken from corrected hysteresis loops [24].............................. 34

Figure 3.3. $M$ vs. $T$ data for the $7.6 \mathrm{~nm}$ LSMO/STO sample measured with $H=50,100,200,500 \mathrm{Oe}$, and $1 \mathrm{kOe}$. Arrows mark the bifurcation temperature $T_{\mathrm{B}}$ separating the $M(\mathrm{FC})$ data (open red circles) from the $M$ (ZFC) data (closed blue circles). $T_{\mathrm{C}}$ is the Curie temperature [24].................................................. 35

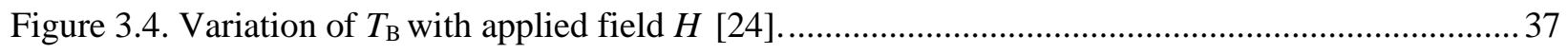

Figure 3.5. The low field zoom of the HL was measured at $190 \mathrm{~K}$ in the ZFC sample of $7.6 \mathrm{~nm} \mathrm{LSMO/STO.}$

Arrows are marked as 1, 2, and 3 to present the counter-clockwise cycle of the loop [69]...................... 38

Figure 3.6. (Left figure) low field zoom in of the HLs for the ZFC sample of $7.6 \mathrm{~nm}$ LSMO/STO at different temperatures (a) $T<T_{\mathrm{B}}$, (b) $T=T_{\mathrm{B}}$, (c) $T_{\mathrm{B}}<T<T_{\mathrm{C}}$, (d) $T=T_{\mathrm{C}}$, (e) $T>T_{\mathrm{C}}$. Arrows are marked as 1,2 , and 
3 to present the direction of the magnetic field sweep. (Right figure) the same data are plotted up to $H=$ \pm 2000 Oe to show loop closing which occurs at about $H=1000$ Oe [69]. .39

Figure 3.7. Temperature variations of $M_{\mathrm{S}}, M_{\mathrm{r}}, H_{\mathrm{C}}, H_{\mathrm{C}}(\mathrm{i})$, and $M_{\mathrm{r}}(\mathrm{i})$ defined in figure 3.5 measured in the sample of $7.6 \mathrm{~nm} \mathrm{LSMO/STO}$. The positions of the blocking temperature $T_{\mathrm{B}}$ and the Curie temperature $T_{\mathrm{C}}$ are marked with vertical lines [69].

Figure 3.8. Variation of the magnetization $M(t)$ with time (the ln scale) at select temperatures after cooling the sample in $H=50$ Oe to the measuring temperature and then switching $H$ to zero. The solid lines are linear fits at higher times to determine viscosity $S$ using the equation: $M(t)=M(0)-S \ln (t)[76] \ldots \ldots . .44$ Figure 3.9. Temperature variations of the magnetic viscosity $S$ and (b) $M$ (2 hours) determined from the analysis of the data in figure 3.8, $M$ (2 hours) being the measured $M$ at the end of 2 hours of time scan. The lines connecting the data points are visual guides [76]. 45 Figure 4.1. (a) Isothermal $M$ versus $H$ curves at selected temperatures from $210 \mathrm{~K}$ to $320 \mathrm{~K}$ in $10 \mathrm{~K}$ steps. (b) Arrott plot of the data at different temperatures yielding $T_{\mathrm{C}} \sim 290 \mathrm{~K}[80]$. .48

Figure 4.2. Magnetic entropy of the sample (open circles) versus temperature at different applied magnetic fields. The solid lines (red and green) are fit the two Gaussian functions and the blue dashed line is the sum of Gaussian functions [80]. 50

Figure 4.3. (a) Magnetic field dependence of the change in magnetic entropy at different temperatures. (b) Temperature dependence of ' $a$ ' and the exponent ' $n$ ' versus temperature [80].

Figure 4.4. Calculated temperature dependence of specific heat of the sample in different magnetic fields from $2 \mathrm{kOe}$ to $4 \mathrm{kOe}$. The line connecting the points are visual guides. .53

Figure 4.5. (a) The calculated relative cooling power (RCP) of the $7.6 \mathrm{~nm}$ LSMO/STO sample versus applied magnetic field at $220 \mathrm{~K}$ and $270 \mathrm{~K}$. (b) Relative volume fractions of the $\operatorname{SPM}(\alpha)$ and FM $(\beta)$ magnetic phases. The lines connecting the data points are visual guides.

Figure 4.6. Magnetic entropy of the sample versus temperature at $H=4 \mathrm{kOe}$. The blue circles are the data and the dashed line connecting the data points is for the visual guide. The solid brown line is the connected curve of the two peaks at $220 \mathrm{~K}$ and $270 \mathrm{~K}$. (For interpretation of the references to color in this figure legend, the reader is referred to the Web version of this article.)

Figure 5.1. (a) Plots of experimental $\chi^{\prime}$ and $\chi^{\prime \prime}$ vs temperature at four frequencies with the inset in the top figure showing an expanded view of the broad peak near $230 \mathrm{~K}$. (b) High-temperature zoom of the $\chi^{\prime}$ and $\chi^{\prime \prime}$ vs temperature data at different frequencies [76].

Figure 5.2. (a) Plots of computed $\mathrm{d} M / \mathrm{d} T$ vs $T$ for different $H$ to determine $T_{\mathrm{C}}$ using the $M$ vs $T$ data of figure 3.3 in section 3.4.2. (b) Magnetic field variation of $T_{\mathrm{C}}$ with the solid purple line fit to the equation-5-11) and the inset showing the plot of $\ln \left[T_{\mathrm{C}}(H)-T_{\mathrm{C}}(0)\right]$ vs $\ln H$ with the red line as the linear fit [76]..... 
Figure 5.3. Plots of experimental $\chi^{\prime}$ and $\chi^{\prime \prime}$ vs temperature for one frequency $f=1 \mathrm{kHz}$ and $H(\mathrm{ac})=10 \mathrm{Oe}$ but for two static fields $H=0$ Oe (blue solid squares) and $H=100$ Oe (red open circles). The data of $\chi^{\prime}$ and $\chi^{\prime \prime}$ for $H=100$ Oe are multiplied by a factor of 5 for visual clarity [76] ...........................................65 Figure 5.4. The Cole-Cole plots at different temperatures from $269 \mathrm{~K}$ to $278 \mathrm{~K}$. The solid red curves are fit to the equation-(5-10) with the parameters of the fits listed in each figure [76].....

Figure 5.5. (a) The best-fit linear line in the plot of $\ln (\tau)$ vs $1 /\left(T-T_{0}\right)$ based on the Vogel-Fulcher law [equation-(3-47)] to determine the $\Delta E / k_{\mathrm{B}}$ and $\tau_{0}$. (b) Shows how the calculated values of $\tau$ from the fit the Vogel-Fulcher law using the parameters determined from the plot in (a) [76]. .68 


\section{List of Tables}

Table 4-1. Summary of the reported RCPs of LSMO systems compared with the data on the $7.6 \mathrm{~nm}$ LSMO/STO film. .56 


\section{Chapter 1 \\ Background and motivation}

\subsection{Introduction}

Thin films and nanoparticles (NPs) have significant surface effects as the thickness of thin films or the diameter of NPs decrease, and the surface to bulk ratio increases. Therefore, the surface contribution in magnetic and electrical properties of these nanostructures is dominant [1,2]. Similar surface effects can happen in thin films. Researchers claim that by decreasing the thickness of the films, some layers or regions do not have any magnetic contributions. These layers/regions are called magnetic dead layers (MDLs) and their contribution in reducing the magnetic and electrical properties of complex oxide films such as $\mathrm{La}_{0.7} \mathrm{Sr}_{0.3} \mathrm{MnO}_{3}$ (LSMO) is significant [2,3]. The MDLs' complications are the main interest of study in this dissertation. For this purpose, LSMO films are chosen.

My main material of choice for this study was thin films of LSMO, which is a material in the manganite group categorized as mixed-valence. This group shows different magnetic phases based on many factors such as the element ratios and fabrication process. We grow our films using a very high-quality pulsed laser deposition technique that allows layer-by-layer growth. Despite the quality of our films, I will demonstrate that the results demonstrate magnetic inhomogeneity. In this chapter, I start my discussion on the basics of magnetism and the common magnetic phases which are present in nature. After, I give a short discussion on the crystal structure of manganites. Then I explain why LSMO is an interesting material in the field of magnetism. Finally, I go through the magnetic studies on LSMO thin films and nanoparticles.

\subsection{A Brief Discussion on the Basics of Magnetism and Magnetic Phases}

In electromagnetism, the magnetic moment $\mu$ is explained by a current $I$ which passes through a loop with the surface of $\mathrm{d} A$. By summing up magnetic moments of the loops the magnetic moment of finite-size is

$$
\mu=I \int \mathrm{d} A \text {. }
$$

To calculate the magnitude of magnetization $(M)$ of a material we need to know the total magnetic moment of the sample per unit of volume. The $M$ can be calculated as

$$
M=\mu \frac{N}{V}
$$

where $N$ and $V$ are the total magnetic moments and the volume of the sample, respectively. If the magnetic material is exposed to the external magnetic field $(H)$ the relationship between $M$ and $H$ is [4] 


$$
M=\chi H,
$$

which $\chi$ is the magnetic susceptibility of the material. This simple explanation is in the classical picture and a more complicated one will be discussed in the quantum mechanical picture of magnetization in section 3.2.1. By knowing the $\chi$ of the material we can define the classification of magnetic materials into three main categories: diamagnetic material, paramagnetic (PM) material, and materials with collective magnetism.

\subsubsection{Diamagnetic Material}

The $\chi$ of a diamagnetic material is constant and negative. If a diamagnetic material is exposed to $H$, the directions of the magnetic dipole are antiparallel with respect to the direction of $H$ due to Lenz's law. Many materials, such as organic materials and superconductors, are classified as diamagnetic materials.

\subsubsection{Paramagnetic Materials}

The $\chi$ of paramagnetic (PM) materials is positive $(\chi>0)$ and by applying $H$ to these materials the magnetic dipoles are oriented in the direction of $H$. Thermal fluctuations can destroy the orientation of $\chi$.

\subsubsection{Collective Magnetism}

The $\chi$ of these magnetic materials is a function of temperature $(T)$ and $H$, and it is more complicated than the $\chi$ of diamagnetic and PM materials. The magnetism in these materials is governed by the exchange interactions between the magnetic dipoles which can be explained by quantum mechanical rules. The orientation of magnetic dipoles is intrinsic, and in the absence of $H$, the dipoles are oriented below the critical temperature $\left(T_{\text {critical }}\right)$ of the material. Magnetic materials in this category can be divided into four groups: ferromagnetic (FM) materials, superparamagnetic (SPM) ferrimagnetic (FIM) materials, and antiferromagnetic (AFM) materials.

\subsubsection{Ferromagnetism}

In FM materials, the magnetization develops below the $T_{\text {critical }}$ of the sample. The $T_{\text {critical }}$ is called the Curie temperature $\left(T_{\mathrm{C}}\right)$. Figure 1.1 presents the temperature dependence of magnetization $(M-T)$ below and above the $T_{\mathrm{C}}$ of the sample. At zero Kelvin temperature $(T=0 \mathrm{~K})$ and in no applied magnetic field $(H=0)$, the direction of all magnetic dipoles of the sample is parallel and the system is ordered. The magnitude of magnetization at $T=0 \mathrm{~K}$ is called saturation magnetization $\left(M_{\mathrm{s}}\right)$. As the temperature of the sample increases thermal fluctuations overcomes the magnetic interactions between magnetic dipoles and magnetic dipoles start to fluctuate. In $T_{\mathrm{C}}<T$, all magnetic moments are randomly oriented, and the magnetic state goes into a disordered state. In this case, the symmetry is broken, and the magnetization in the sample is zero. 


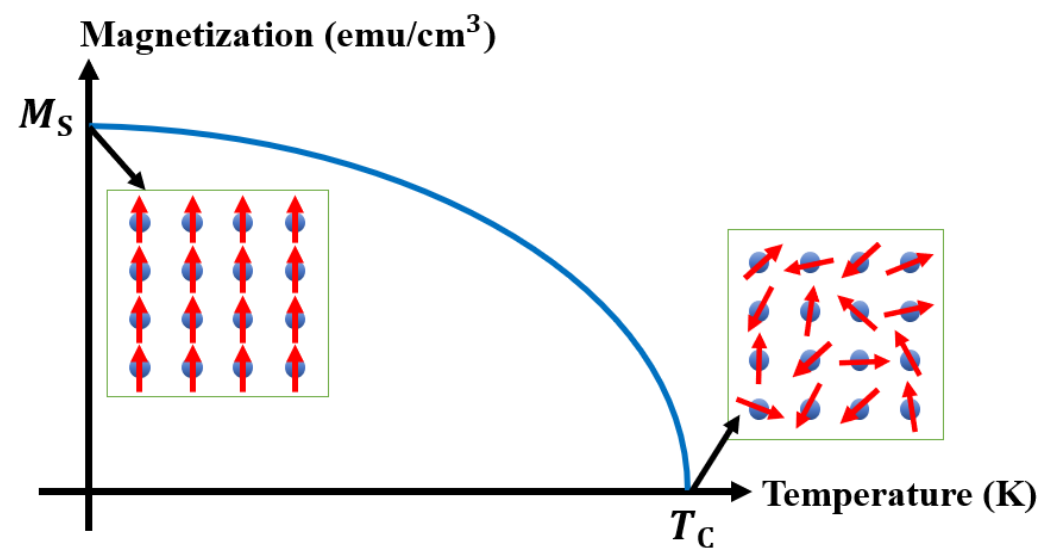

Figure 1.1. $M-T$ plot of FM material.

\subsubsection{Superparamagnetism}

This class of magnetic state emerges in small ferromagnetic nanoparticles consists of a single magnetic domain. At a certain temperature which is called blocking temperature $\left(T_{\mathrm{B}}\right)$, the thermal energy can switch the magnetic moment of the particles from parallel to antiparallel directions with respect to the applied magnetic field and vice versa such that the particle behaves like a PM material and subsequently above $T_{\mathrm{B}}$ the net magnetization is zero. Therefore, these materials do not have hysteresis in comparison with FM materials. I explain the other magnetic characteristics of SPM in chapter 3 such as the energy barrier and magnetic relaxation of this system.

\subsubsection{Antiferromagnetism}

In this type of magnetic state, the AFM material has two identical magnetic sublattices with the same critical temperature known as the Néel temperature $\left(T_{\mathrm{N}}\right)$. Figure 1.2 shows the $M$ - $T$ plot of an AFM material. In $T=0 \mathrm{~K}$ well below $T_{\mathrm{N}}$, both sublattices are magnetized with the same amount but in opposite directions, therefore the magnetization of the sample is zero. In nature, some AFM materials exhibit non zero $M$ at $T$ $\approx 0 \mathrm{~K}$ which is explained as spin canting. The spin canting occurs when antiparallel magnetic moments of the two sublattices are not perfectly aligned and antiparallel respect with to the easy axis and they are tilted from the easy axis which results in $M \neq 0$. At $T_{\mathrm{N}}<T, M-T$ is very similar to PM material and the symmetry is broken. As a result, the magnetic state of the sample is disordered. 


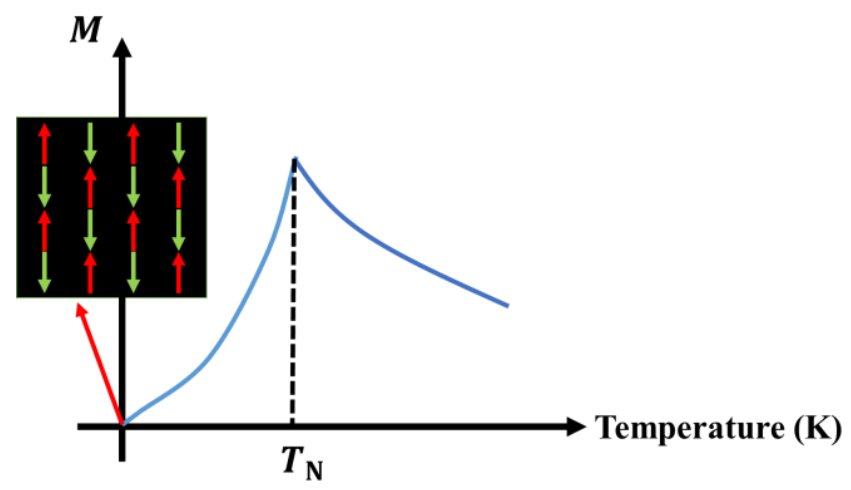

Figure 1.2. $M-T$ plot of a AFM material.

\subsubsection{Ferrimagnetism}

This type of magnetic state is similar to the FM and AFM states. A FIM material shows a spontaneous magnetization below $T_{\mathrm{C}}$ like FM materials. In comparison with FM material, a FIM material has two magnetic sublattices that each have unequal and antiparallel magnetization thus there is a net magnetization $\left(M_{n e t}\right)$, unlike AFM. Figure 1.3 shows one case of $M$ - $T$ of a FIM material. In some FIM materials, at a certain temperature, the $M$ of both sublattices are equal and the net $M$ of the sample is zero. This temperature is called compensation temperature $\left(T_{\text {comp }}\right)$.

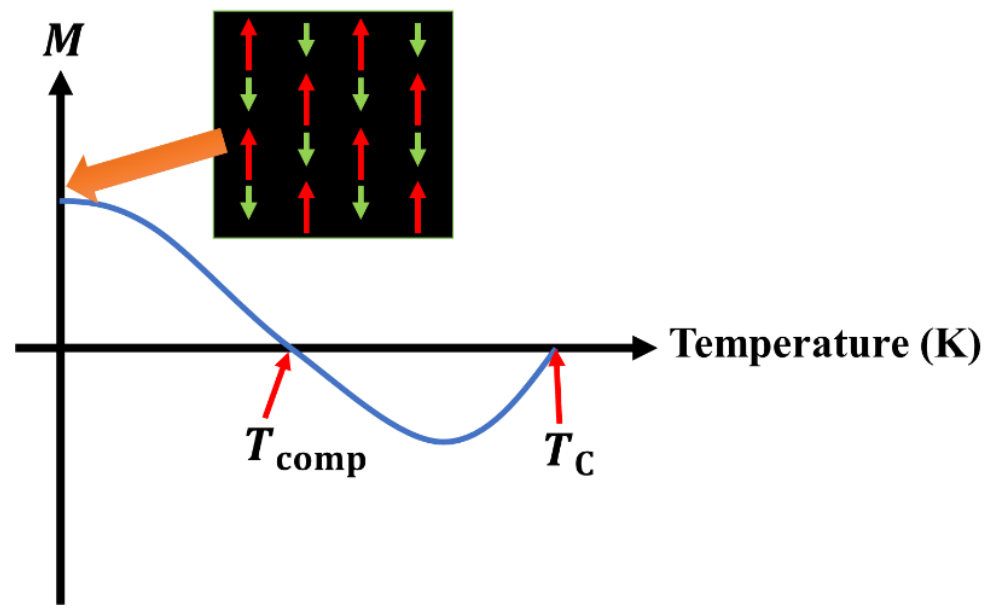

Figure 1.3. $M-T$ plot of a FIM material

So far, I explained the fundamental magnetic structures in nature. The mentioned magnetic phases above are the common phases, but the complexity of nature introduces other magnetic phases such as spin glasses and the Griffiths phase. The Griffiths phase is the existence of short-rang ordering of FM clusters in a PM medium at $T_{\mathrm{C}} \leq T \leq T_{\mathrm{G}}$ which $T_{\mathrm{G}}$ is defined as the characteristic temperature of the FM clusters start to 
nucleate [5]. The spin-glass appears below the freezing temperature $\left(T_{\mathrm{f}}\right)$ which random spins freeze below this temperature in the magnetic medium. This magnetic phase is disordered since the spins are aligned and coupled randomly [6].

As a matter of fact, a magnetic material may contain two or more magnetic phases which depend on the materials. For example, a film can be made of two materials with two different magnetic phases such as FM and AFM phases. In the other scenario, the material such as nanoparticles of a single material can show two different magnetic phases due to their different sizes. The co-existence of mix magnetic phases makes the magnetic sample magnetically inhomogeneous and the magnetic properties of these materials are extremely interesting due to the magnetic phase competition. LSMO films with the optimum ratio of $\mathrm{La} / \mathrm{Sr}$ $\sim 0.3$ are known as FM materials but, in my work, I found these films are not purely FM material and they have another magnetic phase that acts a lot like SPM which reduces the $M_{\mathrm{S}}$ of samples and causes interesting magnetic phenomena which is tunable negative magnetization (TNM). In the next chapters, I will explain the methods of magnetic characterizations to detect the magnetic phases in an LSMO film and the source of TNM.

In the next section, I go through the crystal structure LSMO and the magnetism in this material.

\subsection{Crystal Structure of LSMO}

The story of mixed-valence manganites goes back to the 1950s when G. H. Jonker and J. H. Van Santen fabricated these materials in the form of polycrystals to understand the magnetic properties of manganites [7]. They found that these materials have a perovskite structure with FM properties. Figure 1.4 presents the cubic perovskite structure. Manganese oxide has the general formula of $\operatorname{Re}_{1-\mathrm{x}} \mathrm{T}_{\mathrm{x}} \mathrm{MnO}_{3}$, in which $\mathrm{Re}$ is a trivalent rare earth element, and $\mathrm{T}$ is a divalent alkaline earth element [8]. The $A$ sites and $B$ sites are presented by big blue and small green spheres, respectively, and oxygen sits at the center of the faces of the cube. The Re and T elements are located at the $A$ sites with 12-fold oxygen coordination. Mn cations are at the $B$ sites of the cube which is in the octahedral oxygen coordination. In this perovskite structure, the divalent Re element substitutes the T element which some of $\mathrm{Mn}^{3+}$ convert into $\mathrm{Mn}^{4+}$ to maintain the charge neutrality. The stochiometric fractions of $\mathrm{Mn}^{3+}$ and $\mathrm{Mn}^{4+}$ are 1-x and $\mathrm{x}$, respectively.

It is reported that the lattice parameter of LSMO films changes due to lattice mismatch between the films and the STO substrate which changes the cubic structure of the LSMO into a pseudocubic structure. The pseudocubic structure has the lattice parameters as $a=b=3.905 \AA$ and $c=3.846 \AA$ with $\alpha=\beta=90^{\circ}$ and $\gamma=90.87^{\circ}[9,10]$. 


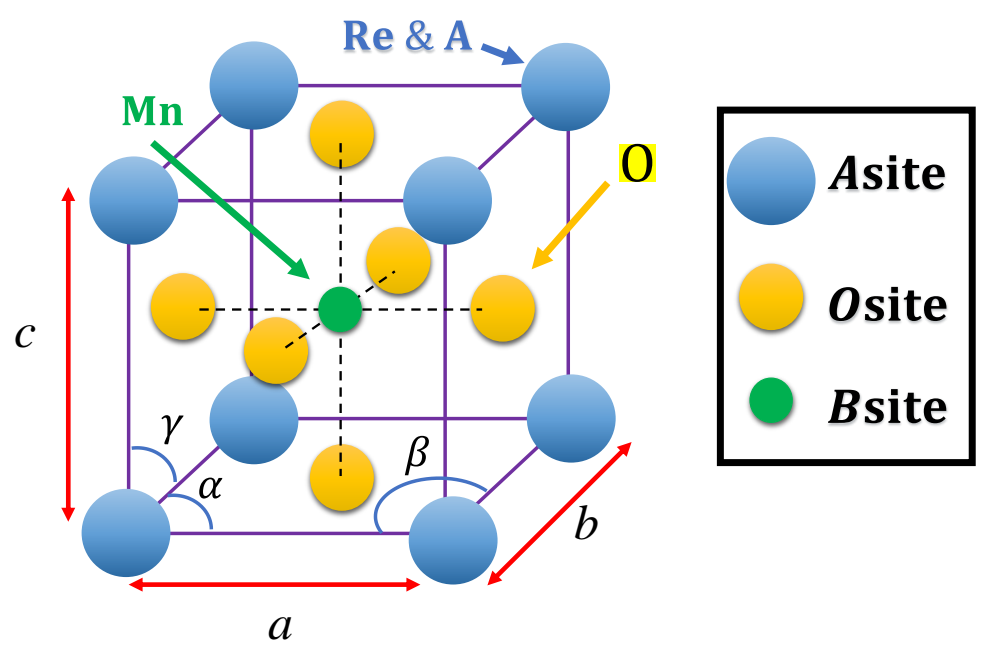

Figure 1.4 Cubic Perovskite Structure.

The $\mathrm{Mn}$ atom has the electronic configuration as $1 \mathrm{~s}^{2} 2 \mathrm{~s}^{2} 2 \mathrm{p}^{6} 3 \mathrm{~s}^{2} 3 \mathrm{p}^{6} 3 \mathrm{~d}^{5} 4 \mathrm{~s}^{2}$ which the $3 d$ orbital is halffilled. Due to the crystal field from the oxygen atoms, the 5-fold orbital degeneracy of the $3 d$ orbitals breaks into three $t_{2 \mathrm{~g}}$ and two $e_{\mathrm{g}}$ degenerate states. The $t_{2 \mathrm{~g}}$ states lie in between cartesian coordinate system point between the ligands, on the other hand, the $e_{\mathrm{g}}$ states point directly at the ligands thus the $t_{2 \mathrm{~g}}$ experience less coulomb repulsion than $e_{\mathrm{g}}$ states, and subsequently, $t_{2 \mathrm{~g}}$ states have the lower energies with respect to the $e_{\mathrm{g}}$ states. The electrons fill the orbital states of $\mathrm{Mn}^{3+}$ and $\mathrm{Mn}^{4+}$ by Hund's first rule which the maximum value of spin $(S)$ must be satisfied. Therefore, the $S$ of $\mathrm{Mn}^{3+}$ and $\mathrm{Mn}^{4+}$ is 2 and 3/2, respectively. The $t_{2 \mathrm{~g}}$ level of $\mathrm{Mn}^{4+}$ is filled with three electrons whereas the $e_{\mathrm{g}}$ has no electrons. This electron configuration of Mn cations embedded in octahedral oxygen coordination governs the magnetic and electrical properties of manganites [8].

\subsubsection{Magnetic Behavior in Manganites}

The magnetic behavior of the manganites can be described by the double-exchange model proposed by Zener [11]. The proposed mechanism is based on the indirect coupling between the $d$-orbitals of manganese cations. In this mechanism the two $\mathrm{Mn}$ cations, $\mathrm{Mn}^{3+}$ and $\mathrm{Mn}^{4+}$ are separated by an oxygen atom, unlike the super-exchange mechanism where the two nearest magnetic ionic neighbors are Mn cations with the same valence $[12,13]$. There are two possible degenerate states of Mn cations in the double-exchange. The states are [14]

$$
\Psi_{1}: \mathrm{Mn}^{3+} \mathrm{O}^{2-} \mathrm{Mn}^{4+} \text {, and } \Psi_{2}: \mathrm{Mn}^{4+} \mathrm{O}^{2-} \mathrm{Mn}^{3+} \text {. }
$$


In $\Psi_{1}$, an electron hops from the $2 p$ orbital of $\mathrm{O}^{2-}$ to the empty $e_{\mathrm{g}}$ orbital of $\mathrm{Mn}^{4+}$ and simultaneously another electron hops from $\mathrm{Mn}^{3+}$ to the $2 p$ orbital of $\mathrm{O}^{2-}$ which lowers the energy of this orbital, and $\Psi_{1}$ changes into the new state, $\Psi_{2}$. The magnetic system resonates between these two degenerate states. Figure 1.5 shows a simple picture of the electron hopping from $\mathrm{O}^{2-}$ to $\mathrm{Mn}^{4+}$ and from the $\mathrm{Mn}^{3+}$ to $\mathrm{O}^{2-}$. According to Hund's rule, the hopped electron must have the same spin as the other electrons and all the magnetic moments on both Mn cations are parallel. This creates the lowest energy states, and it is leading to a FM ground state.

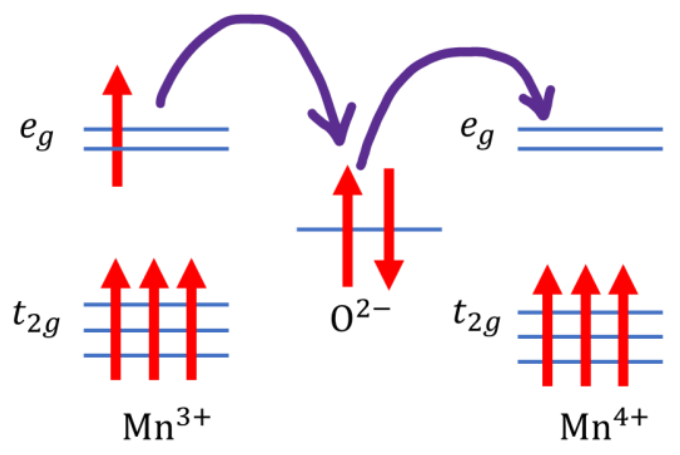

Figure 1.5 Double-exchange between the Mn cations $[8,14]$.

The double-exchange mechanism in $\mathrm{Re}_{1-\mathrm{x}} \mathrm{A}_{\mathrm{x}} \mathrm{MnO}_{3}$ compounds is well understood. By changing the composition of these compounds, we can achieve different magnetic phases and electronic properties. Santen and Jonker proved that the $T_{\mathrm{C}}$ of these materials depends on the doping level $\mathrm{x}$ and the average ionic radius of the cation in the A position [7,15]. They showed the highest $T_{\mathrm{C}}$ and $M_{\mathrm{s}}$ value is achievable in $\mathrm{La}_{1-\mathrm{x}} \mathrm{Sr}_{\mathrm{x}} \mathrm{MnO}_{3}$ with the optimum ratio of $\mathrm{x} \sim 0.3 . \mathrm{La}_{0.7} \mathrm{Sr}_{0.3} \mathrm{MnO}_{3}$ (LSMO) films are good candidates for spintronics [16], multiferroic tunnel junctions [17], colossal magnetoresistance [18], resistive switching memory devices [19], ferroelectric/ferromagnetic systems [20], and heterostructures [21]. Although LSMO films have many applications, there is a barrier to achieve the most magnetic efficiency in LSMO thin films, which is the presence of MDLs. As discussed earlier in this chapter the magnetic thin films and nanoparticles suffer from surface effects. The surface effects create MDLs which change the magnetic and electrical properties of these nanostructures. The research on MDLs is vast, and I discuss the recent and past discussions on this matter especially in the LSMO system.

\subsection{Studies on LSMO Nanoparticles and Thin Films to Understand the Dead Layers}

Studies on manganite oxide films and NPs show that the magnetic properties of these materials are affected by MDLs [22,23]. In 2009, Curaile et al. studied the magnetization of $\mathrm{La}_{0.67} \mathrm{Sr}_{0.33} \mathrm{MnO}_{3} \mathrm{NPs}_{\text {and }}$ $\mathrm{La}_{0.67} \mathrm{Ca}_{0.33} \mathrm{MnO}_{3}$ (LCMO) nanotubes (NTs) by the dc bulk magnetometry measurements [22]. The $M-T$ measurements of NPs and NTs in zero-field-cooled (ZFC) and field-cooled (FC) cycles showed that the $M_{\mathrm{S}}$ 
was reduced $50 \%$ compared with the $M_{\mathrm{S}}$ of the bulk. Figure 1.6-(c) shows the ZFC and FC $M-T$ plots. They used high-resolution transmission electron microscopy (HTEM) measurements to study the structure of NPs and NTs. They found that the MDLs are at the surface of these structures. The thickness of the surface layer is about $2 \mathrm{~nm}$ and this layer does not have long-range crystalline order. Figure 1.6-(a) and (b) shows the HRTEM of a LCMO NT and a LSMO NP, respectively, with the surface layers around the NT and NP. The structural distortions in the surface layer destroy the double-exchange interactions between Mn cations which result in depletion of FM in this layer [22].
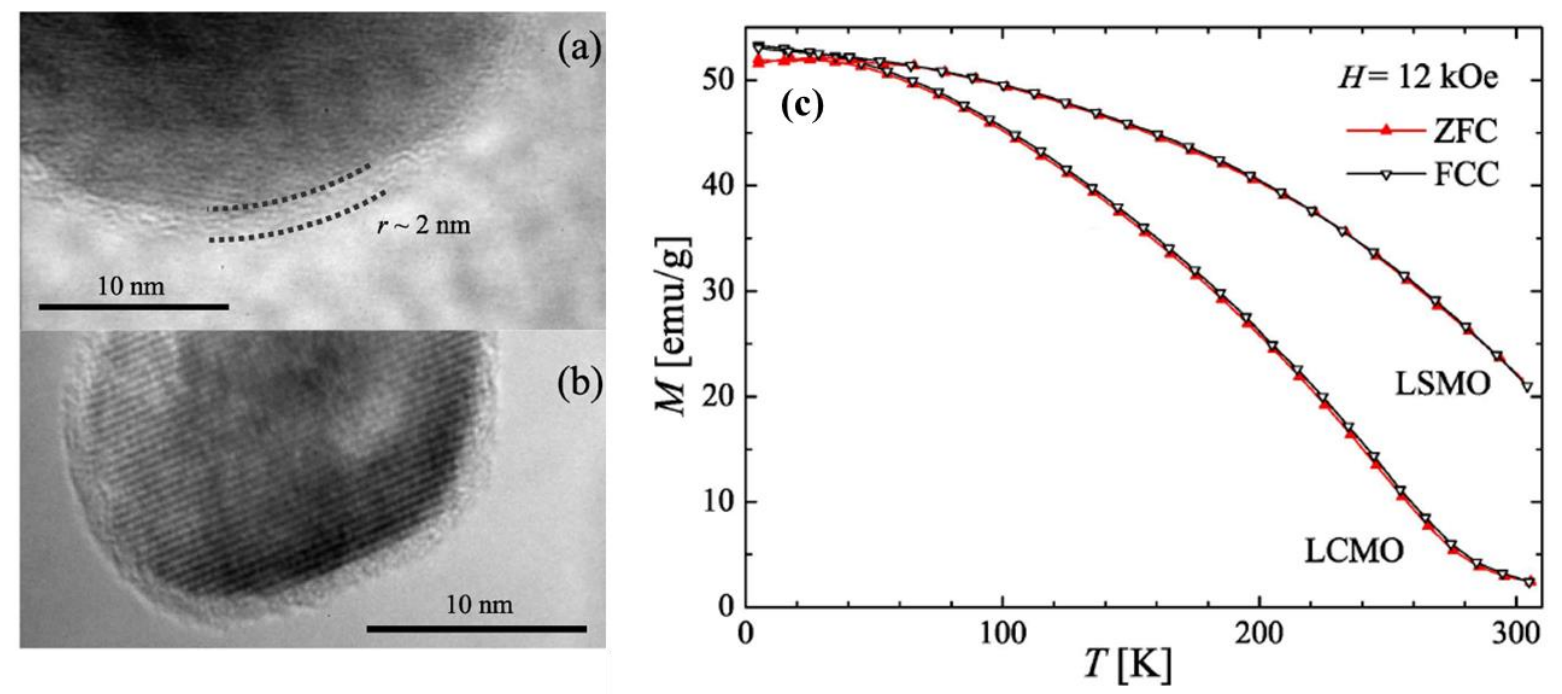

Figure 1.6 HRTEM of (a) LCMO NT and (b) LSMO NP. The surface layer with the thickness of $2 \mathrm{~nm}$ is indicated by a dotted line. (c) $M-T$ measurements of LCMO NTs and LSMO NPs in ZFC and FC cycles measured at $H=12 \mathrm{kOe}$. Reproduced from, [22] with the permission of AIP Publishing.

The MDLs are not only in NPs and they have been observed in thin films. Huijben et al. performed magnetic and transport measurements on LSMO thin films with various thicknesses between 3 u.c to 70 u.c. $(1$ u.c. $=0.4 \mathrm{~nm})$, fabricated by pulsed laser deposition (PLD), at different oxygen pressures [3]. They observed the reduction of $M_{\mathrm{S}}$ in thin films which were grown in lower oxygen pressures (Figure 1.7-a). They measured the temperature variation of magnetization of all samples by cooling them in 1 Tesla applied field and subsequently measured the magnetization in 100 Oe applied field in warming upcycle. The reduction of $M_{\mathrm{S}}$ in lower oxygen pressures was due to oxygen vacancies (OVs). As discussed in section 1.3 , the double-exchange mechanism is caused by the hopping of an electron from $\mathrm{Mn}^{3+}$ to $\mathrm{Mn}^{4+}$ mediated by oxygen anions. OVs break the double-exchange interaction between Mn cations and it diminishes the FM ordering.

They also studied the thickness effects on the $M_{\mathrm{S}}$ value of the films by growing the samples in the optimum oxygen pressure at 200 mTorr oxygen pressure. Figure 1.7-b shows the $M-T$ plots of the films. 
The $M_{\mathrm{S}}$ reduces by decreasing the thickness of the film and it vanishes in 3 u.c. film thickness. By decreasing the thickness of the film, the number of nearest magnetic ions reduces, and subsequently, the number of double-exchange interactions goes down in the sample. The reported thickness of the MDL in LSMO films grown at 200 mTorr is 3 u.c. $(\sim 1.2 \mathrm{~nm})$ [3].
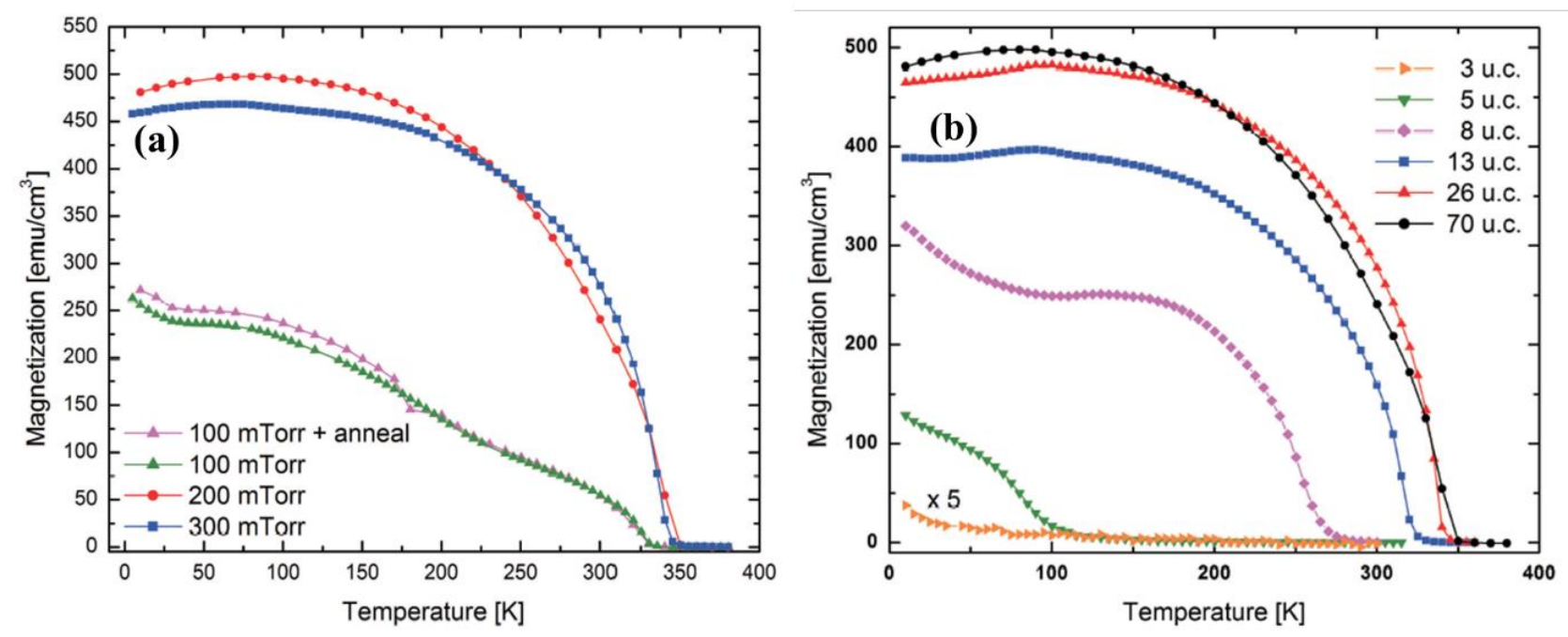

Figure 1.7 M-T plots of LSMO films (a) grown at different oxygen pressures, (b) with different thicknesses. Reproduced from [3] with the permission of APS Publishing.

To understand the OVs and thickness effects on $M_{\mathrm{S}}$ and $T_{\mathrm{C}}$ of the LSMO films, I used the available data from the literature on LSMO thin films prepared in 200, 150, and $0.38 \mathrm{mTorr}_{2}$ deposition pressures [24]. Each point was accurately determined using the internet available software 'WebPlotDigitizer'1. The thickness variation of $M_{\mathrm{S}}$ and $T_{\mathrm{C}}$ is presented in Figure 1.8. The thickness variation of $M_{\mathrm{S}}$ is modeled by

$$
M_{\mathrm{S}}(D)=M_{\mathrm{S}}(\mathrm{b})(1-d / D)
$$

where $M_{\mathrm{S}}(\mathrm{D})$ and $M_{\mathrm{S}}(\mathrm{b})$ are the $M_{\mathrm{S}}$ values of the active layer and the bulk, respectively. $D$ is the thickness of the film and $d$ is the dead layer thickness. The data fit the model well, showing that with a decrease in $\mathrm{O}_{2}$ pressure, $M_{\mathrm{S}}$ is lowered and $d$ increases. As discussed above, the magnetic properties of manganites are governed by the double-exchange interaction between $d$-orbitals of magnetic $\mathrm{Mn}^{3+}$ and $\mathrm{Mn}^{4+}$ ions with charge transfer from $\mathrm{Mn}^{3+}$ and $\mathrm{Mn}^{4+}$ ions through oxygen anions. Low $\mathrm{O}_{2}$ pressure increases oxygen vacancies, resulting in the breakdown of the exchange mechanism and increased spin disorder which in turn decreases $M_{\mathrm{S}}$ and $T_{\mathrm{C}}$. Also, by decreasing the thickness of the films, the number of nearest neighbors of magnetic ions reduces, and as a result, the $M_{\mathrm{S}}$, and $T_{\mathrm{C}}$ values decrease.

\footnotetext{
${ }^{1}$ https://automeris.io/WebPlotDigitizer/
} 
The variation of $T_{\mathrm{C}}$ with film thickness $D$ of LSMO films is modeled by the finite-size scaling relation:

$$
T_{\mathrm{C}}(D)=T_{\mathrm{C}}(\infty)\left(1-\left(\xi_{0} / D\right)^{\lambda}\right)
$$

where $T_{\mathrm{C}}(D)$ and $T_{\mathrm{C}}(\infty)$ are respectively the Curie temperatures for a film with thickness $D$ and very thick films, $\lambda$ is the shift exponent and $\xi_{0}$ is characteristic microscopic correlation length [25]. The fit of the data to the equation-(1-6) is shown in Figure 1.8-b for the films grown in different $\mathrm{O}_{2}$ pressures with the parameters obtained from the fit listed in the figure. The value of $\lambda$ depends on the theoretical model, $\lambda=$ 2 is obtained from Monte-Carlo simulation [26].

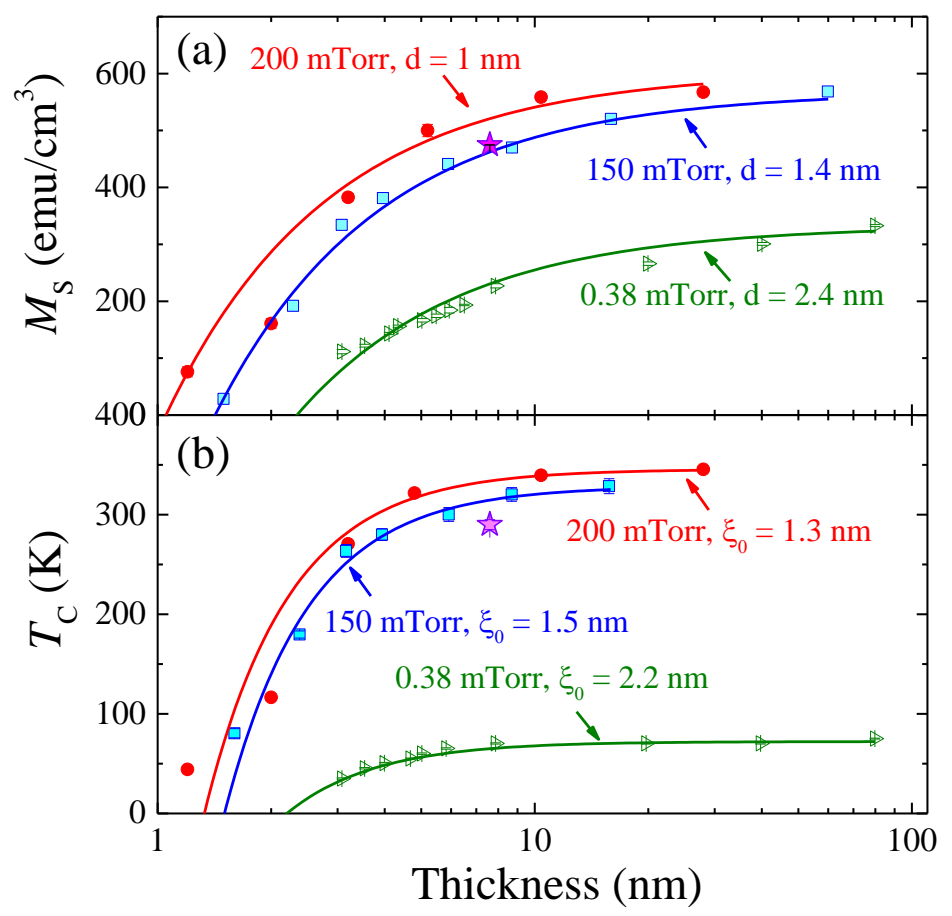

Figure 1.8. Variation of the $M_{\mathrm{S}}$ and $T_{\mathrm{C}}$ with thickness $D$ of the LSMO films. Different symbols represent data from different groups as follows: solid blue rectangle [3], solid red circle [27], open green diamond [23]. The solid lines are fit to the equation-(1-5) in (a) and equation-(1-6) in (b) with the listed parameters.

\subsection{Motivation for this Research and Outline of Approaches}

Understanding the MDLs effect in changing the magnetic properties of thin films is the motivation in my research. As I mentioned earlier, the MDLs show significant effects when the surface-to-volume ratio is higher, and especially in manganite films; MDLs are considered as big barriers in reducing magnetic properties such as the $M_{\mathrm{S}}, T_{\mathrm{C}}$, etc. Therefore, I choose LSMO films to study the surface effects and MDLs. In this work, I found the MDLs are not dead, and they have different magnetic properties than the bulk of the material. The method in this dissertation can be used in many other magnetic systems to detect the 
magnetic phases of MDLs in other thin films. In this study, the accurate measuring of the nature of magnetism of MDL in LSMO films is performed.

To investigate the effects of the MDLs in LSMO films, more detailed dc magnetic measurements were done to study the temperature and field variation of magnetization. The majority of dc measurements were carried out at West Virginia University using the commercial physical property measurement system (PPMS manufactured by Quantum Design Inc) of the magnetic measurements covering the temperature from $5 \mathrm{~K}$ to $400 \mathrm{~K}$ and magnetic field up to $4 \mathrm{kOe}$. $M$ - $T$ measurements were performed in the temperature range from $5 \mathrm{~K}$ to $400 \mathrm{~K}$ in low and high applied magnetic fields from 50 Oe to $4 \mathrm{kOe}$ in zero-field-cooled (ZFC) and field-cooled (FC) cycles.

The presence of blocking temperature $\left(T_{\mathrm{B}}\right)$ defined as the bifurcation temperature between ZFC and FC cycles of $M$ vs. $T$, is indicative of blocked spin clusters which cause the magnetic phase competition between spin clusters and FM phase in a LSMO sample leading into TNM. The observation of TNM and the presence of magnetic phases in a $7.6 \mathrm{~nm}$ LSMO/STO sample proved that this sample is magnetically inhomogeneous. The field variation of magnetization (hysteresis loops) was done in the temperature range of $5 \mathrm{~K}$ to $400 \mathrm{~K}$ in ZFC and FC cycles as well. The hysteresis loops show the hysteresis loop inversion between the two magnetic phase transition temperatures at $T_{\mathrm{B}}$ and $T_{\mathrm{C}}$, which is the confirmation of my observation on $M-T$ measurements.

In this work, the dc measurements are not only limited to $M-T$, and hysteresis loop measurements, the isothermal measurements of $M$ versus $H$ are used to determine the magnetocaloric properties of an inhomogeneous sample, $7.6 \mathrm{~nm}$ LSMO/STO thin film. The two broad peaks in the magnetic entropy vs. $T$ data of this sample confirmed the presence of both magnetic phases. Moreover, the volume fractions of $\mathrm{SPM} / \mathrm{FM}$ are found to be $17 \% / 18 \%$ which is within $2 \%$ agrees with our calculation on $M_{\mathrm{S}}$ from hysteresis loop measurements. The relative cooling power of the film is also calculated, and it is shown that the nanosize effects reduce the relative cooling power (RCP) in the sample.

Beyond dc magnetic measurements, I used ac magnetic susceptibility to detect the magnetic phases in LSMO samples. The ac magnetic susceptibility measurements, $\chi^{\prime}$ and $\chi^{\prime \prime}$, are in the frequency range of 1 $\mathrm{Hz}$ to $10 \mathrm{kHz}$ were performed by using the PPMS from Quantum Design Inc, not only at University of Connecticut and, but also at National High Magnetic Field Laboratory (NHFML) at Florida State University in Tallahassee, FL. The DC applied magnetic field range was from 10 Oe to 1000 Oe applied field.

The ac magnetic susceptibility is a versatile technique in which we can detect different magnetic phases such as spin-glasses [28], superparamagnetism [29], and superconductivity [30] in magnetic materials. In this technique, we can also study the time dynamics of a magnetic system. In higher frequencies, the $M$ lags 
behind the applied magnetic field, and the relaxation process occurs. The studies of ac susceptibility on LSMO films in this dissertation are new, and it has not been done in any other magnetic films yet. The results are revealing, showing a strong interaction between the spin clusters and FM magnetic phases in a $7.6 \mathrm{~nm}$ LSMO/STO film. The mean relaxation time is calculated from the Cole-Cole analysis from the temperature-dependent $\chi^{\prime}$ and $\chi^{\prime \prime}$.

\subsection{Dissertation Outline}

In chapter 2, I discuss the fabrication process of LSMO thin films grown on STO substrates by pulsed laser deposition, and I explain the structural characterization techniques such as X-ray diffraction and Xray reflectivity. In chapter 3, I outline theoretical and experimental approaches to describe the inhomogeneous magnetic system, LSMO films. At the beginning of chapter 3, I cover the theory of magnetism related to a FM system and including the Heisenberg exchange interaction, Spin Wave theory, and, the magnetic viscosity to describe the SPM phase. After the theoretical section, I go over experimental measurements which are mainly the dc bulk magnetometry measurements. In this chapter, I also explain an interesting phenomenon which is the tunable negative magnetization (TNM) occurring due to the presence of MDLs causing magnetic phase competitions in LSMO films.

In chapter 4, I explain the magnetocaloric measurements on a $7.6 \mathrm{~nm} \mathrm{LSMO} / \mathrm{STO}$ sample to detect the magnetic phase transitions in this sample which this chapter has theoretical and experimental work. In chapter 5, I present the detailed measurements of ac bulk magnetometry measurements on a $7.6 \mathrm{~nm}$ LSMO/STO sample. The spin dynamics of an inhomogeneous LSMO/STO film are studied by Cole-Cole analysis for the first time to calculate the mean relaxation time. I give a summary of the unique results on LSMO/STO films from this work along with suggestions for future studies in chapter 6. 


\section{Chapter 2 \\ Thin Films Fabrication Process, Structural and Magnetic Characterization of LSMO Films}

\subsection{Introduction}

The pulsed laser deposition is a famous fabrication technique among other material synthesis processes in metal oxide films, specially LSMO films. To grow a high-quality film by this technique requires significant preparation and verification. In this chapter, I discuss the fabrication process and structural characterization of LSMO films. As the films are deposited on $\mathrm{SrTiO}_{3}$ substrates, I explain the substrate treatment here. All films are fabricated by Pulsed Laser Deposition (PLD) technique thus I give a brief discussion on this fabrication technique. The structural characterization and thickness measurements of LSMO films are determined by X-ray diffraction and X-ray reflectivity and I explain both methods of characterizations here.

\subsubsection{Pulsed Laser Deposition Technique}

The invention of pulsed laser deposition (PLD) goes back to the 1960s. PLD is a physical vapor deposition process that is carried out in a vacuum system down to ultrahigh vacuum (UHV) conditions. Figure 2.1 presents a basic schematic of a PLD system. In this system, the laser pulses produce the plasma of a material to be deposited on a substrate. The laser beam is directed by optical lenses to the surface of the target. The target and substrate are placed on the rotational target and substrate holders, respectively in a vacuum chamber. The gas and pump valves are connected to the chamber to insert and control the level of a gas background. The reflection high-energy electron diffraction (RHEED) is attached to this chamber to monitor the growth.

To fabricate a film from the target, the laser beam must be focused on a small volume of a target. Due to the high energy density absorption of the target, thermal evaporation occurs. The created vapor absorbs the laser energy which continues until a dense plasma is formed. The dense plasma is called a plume. The plume consists of high-energy atomic, diatomic, and other low-mass species of a target. All the species in the plume have collisions near the surface of the target leading to a well-oriented plume perpendicular to the surface of the target. The deposited material on the substrate has the same composition as the target material. The plume material is highly energetic which can react with the gas environment. The gas can be oxygen, nitrogen, and hydrogen to form oxides, nitrides, and hydrides, respectively. 


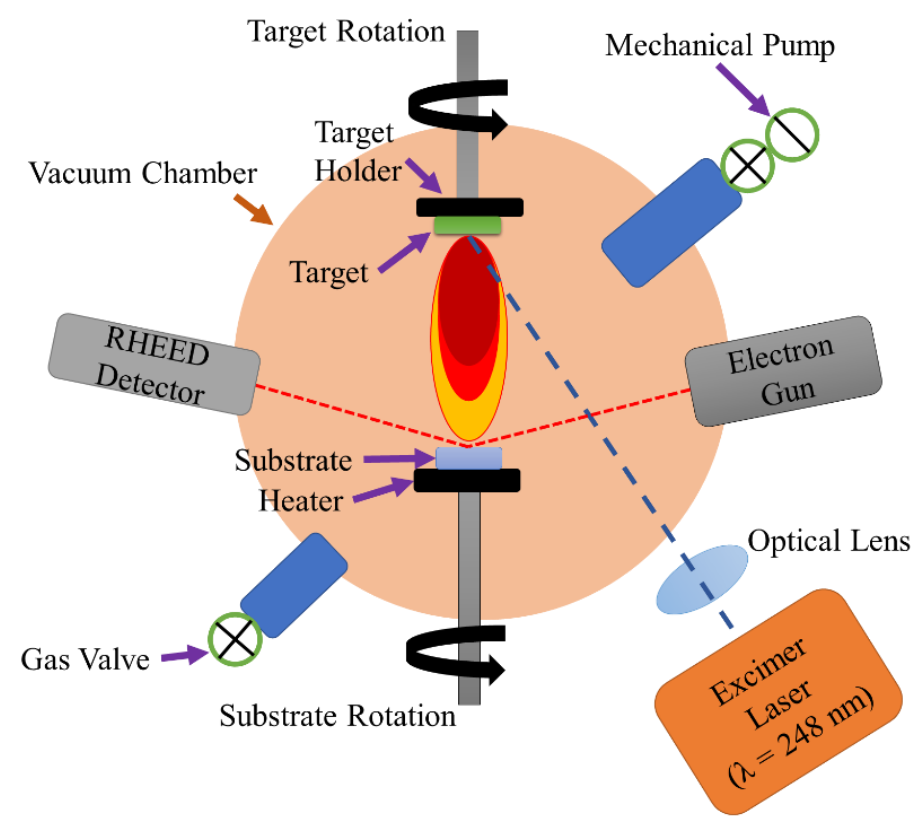

Figure 2.1 Top view of a basic PLD system.

\subsection{Fabrication of LSMO Films}

All LSMO films have been fabricated via PLD with a stoichiometric $\mathrm{La}_{0.7} \mathrm{Sr}_{0.3} \mathrm{MnO}_{3}$ target. The choice of the substrate was $\mathrm{SrTiO}_{3}$ (STO-100) which should be $\mathrm{TiO}_{2}$-terminated. To achieve $\mathrm{TiO}_{2}$ termination, it needs treatment. In the following, I explain why STO is chosen and how the substrate treatment was performed.

\subsubsection{Substrate Treatment}

To obtain an epitaxial and layer-by-layer growth, the substrate must have little to no lattice mismatch, and chemical compatibility with the deposited material thus the choice of a substrate is important in the growth process [31]. The deposited material and the substrate should ideally have similar crystal structures. As it was discussed earlier in section 1.3, the crystal structure of LSMO is a perovskite structure and its film has a unit cell size of $3.889(\AA)$ [3]. The material with the closest unit cell size and similar structure to LSMO is STO with the lattice parameter of $3.905(\AA)$ [32,33]. Figure 2.2 shows the crystal structure of STO. Crystal structure and lattice parameter similarities, cost, and low lattice mismatch make STO and an excellent candidate for the growth process $[27,31]$.

The control over the first layer of the deposited film is important to achieve a smooth film and layer-bylayer growth in the PLD fabrication process. The choice of STO termination can create different physical and chemical properties of LSMO films [35]. STO has two possible terminations in the (001) direction which are made up of alternating layers: $\mathrm{SrO}$ and $\mathrm{TiO}_{2}$ layers (Figure 2.2-b and c). It is shown that to obtain 
a smooth and epitaxial film, a single-terminated substrate is preferable [36]. Among the termination choices, $\mathrm{TiO}_{2}$ is a better choice for LSMO growth since Ti has a smaller coordination number compared with Sr. The coordination number of Ti in bulk is 6 and on the surface is 5 , on the other hand, Sr has 12 and 8 coordination number in the bulk and on the surface, respectively. Because of the Ti coordination number, the $\mathrm{TiO}_{2}$ surface is more stable since fewer atomic bonds break at the surface [36]. Also, the layer-by-layer growth process requires step surfaces on the substrate which the $\mathrm{TiO}_{2}$ layer has the step terrace structure with the height of $4(\AA)$.

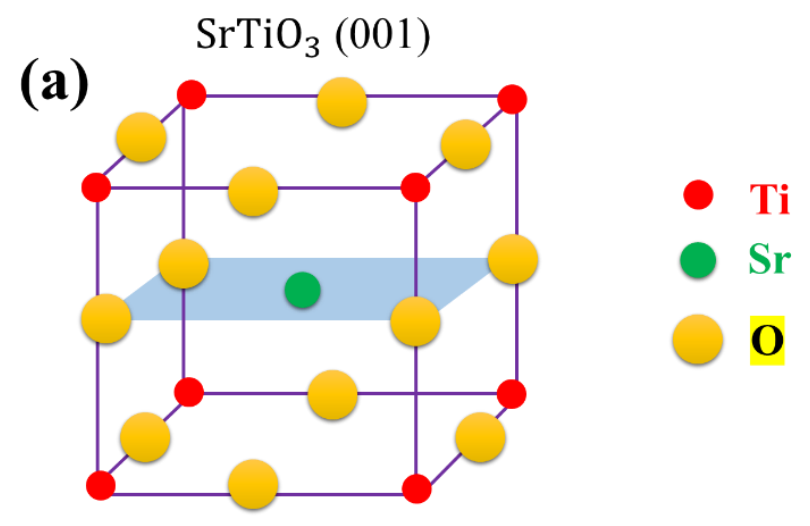

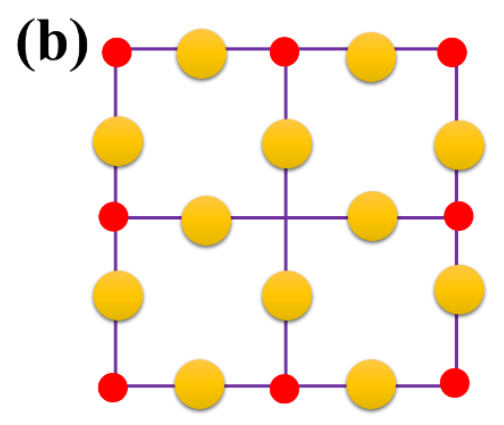

$\mathrm{TiO}_{2}$ plane (c)

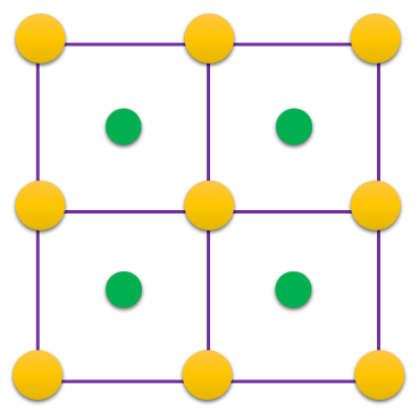

SrO plane

Figure 2.2 (a) cubic structure of $\mathrm{SrTiO}_{3}$, (b) $\mathrm{TiO}_{2}$ plane, (c) $\mathrm{SrO}$ plane.

The treatment process starts by sonicating the as-received STO substrates in acetone for 5 minutes then in isopropanol for 5 minutes to remove any organic and other contaminants on the surface. After the sonication process, the substrates were thermally annealed at $1000{ }^{\circ} \mathrm{C}$ for an hour that $\mathrm{SrO}$ surface segregation was formed by annealing the substrate at high temperature [37,38]. To obtain a single $\mathrm{TiO}_{2}$ termination layer, the annealed substrates were sonicated in DI water for 5 minutes to remove the SrO layer. $\mathrm{SrO}$ is a basic oxide with the metal oxidation state $<+4$, which is dissociated with water to form the $\mathrm{Sr}-$ hydroxide complex [34]. There are other surface treatment methods such as buffered $\left(\mathrm{NH}_{4} \mathrm{~F}-\mathrm{HF}\right) \mathrm{BHF}$ 
solutions [39], $\mathrm{HCl}+\mathrm{HNO}_{3}$ [40], and microwave-induced hydrothermal process [34]. The DI water sonication method is safe and inexpensive compared with the buffered solutions.

To examine the surface of the substrates, atomic force microscopy (AFM) was used. Figure 2.3 presents the AFM height and line profile of a substrate. The treated substrates have the step terrace structure with the height of $4(\AA)$ which is the signature of the $\mathrm{TiO}_{2}$ single layer [41]. The line profile is drawn across three-step heights which is shown by a black solid line in Figure 2.3-a. The line profile is used to measure the height of each step of terraces.

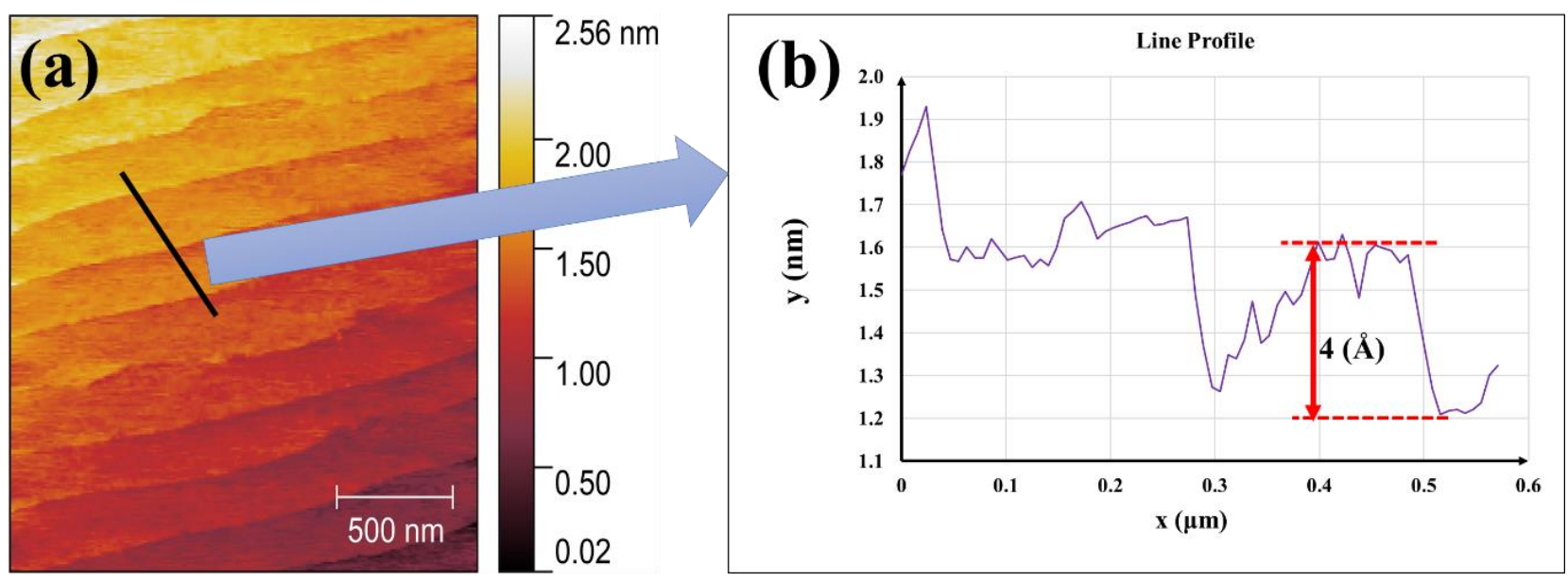

Figure 2.3. (a) AFM image of height, (b) line profile which is shown by a black solid line.

\subsubsection{Fabrication Process and Parameters}

As discussed in section 2.2.1, the $\mathrm{TiO}_{2}$-terminated STO (001) was the choice of the substrate. The film was grown by PLD using a commercial Neocera PLD system with a $248 \mathrm{~nm} \mathrm{KrF}$ excimer laser. The substrate temperature during the PLD growth process was $750{ }^{\circ} \mathrm{C}$ and the chamber was held at 100 mTorr $\mathrm{O}_{2}$ pressure. The laser energy density was $3.1 \mathrm{~J} / \mathrm{cm}^{2}$ with a repetition rate of $5 \mathrm{~Hz}$. Following growth, the thin-films were cooled down to $23{ }^{\circ} \mathrm{C}$ with a $15{ }^{\circ} \mathrm{C} / \mathrm{min}$ rate at $250 \mathrm{mTorr} \mathrm{O}_{2}$ pressure to improve the oxidation quality. The recipe for film growth was optimized via analysis of the KSA reflection high-energy electron diffraction (RHEED) system. The glancing angle of RHEED is around $1^{0}$ to $2^{0}$, which limits the penetration depth of electron in the sample, therefore only the first few layers contribute to diffraction patterns which makes it a suitable instrument as a sensitive real-time in situ technique to surface structure down to a monolayer $[37,38]$. In RHEED the diffraction mechanism is similar to bulk materials but in the film growth process only the first few layers contribute to the diffraction pattern and there are no diffraction conditions perpendicular to the sample surface [44]. 
The reciprocal lattice of the surface of the film consists of infinite rods perpendicular to the surface of a film which are regularly spaced arrays with spacing $2 \pi / a$ and $2 \pi / b$ in $a$ and $b$ directions, respectively. In RHEED the incident electron beam $\overrightarrow{k_{l}}$ hits the surface of the film. In Figure 2.4, the sample sits at the center of Ewald's sphere. The Ewald's sphere is formed when the center of the sphere locates on the surface of the film with a radius of $\overrightarrow{k_{l}}$. The diffraction pattern appears when the rods of reciprocal lattice intersect with Ewald's sphere meaning the difference between the momentum of the incident electron beam $\overrightarrow{k_{l}}$, and the diffracted beam $\overrightarrow{k_{h l}}$ equals to the reciprocal lattice vector $\vec{G}$ [43]. The diffraction spots lie on the circumference of the Laue circles. The diffraction spots are shown with star symbols in Figure 2.4.

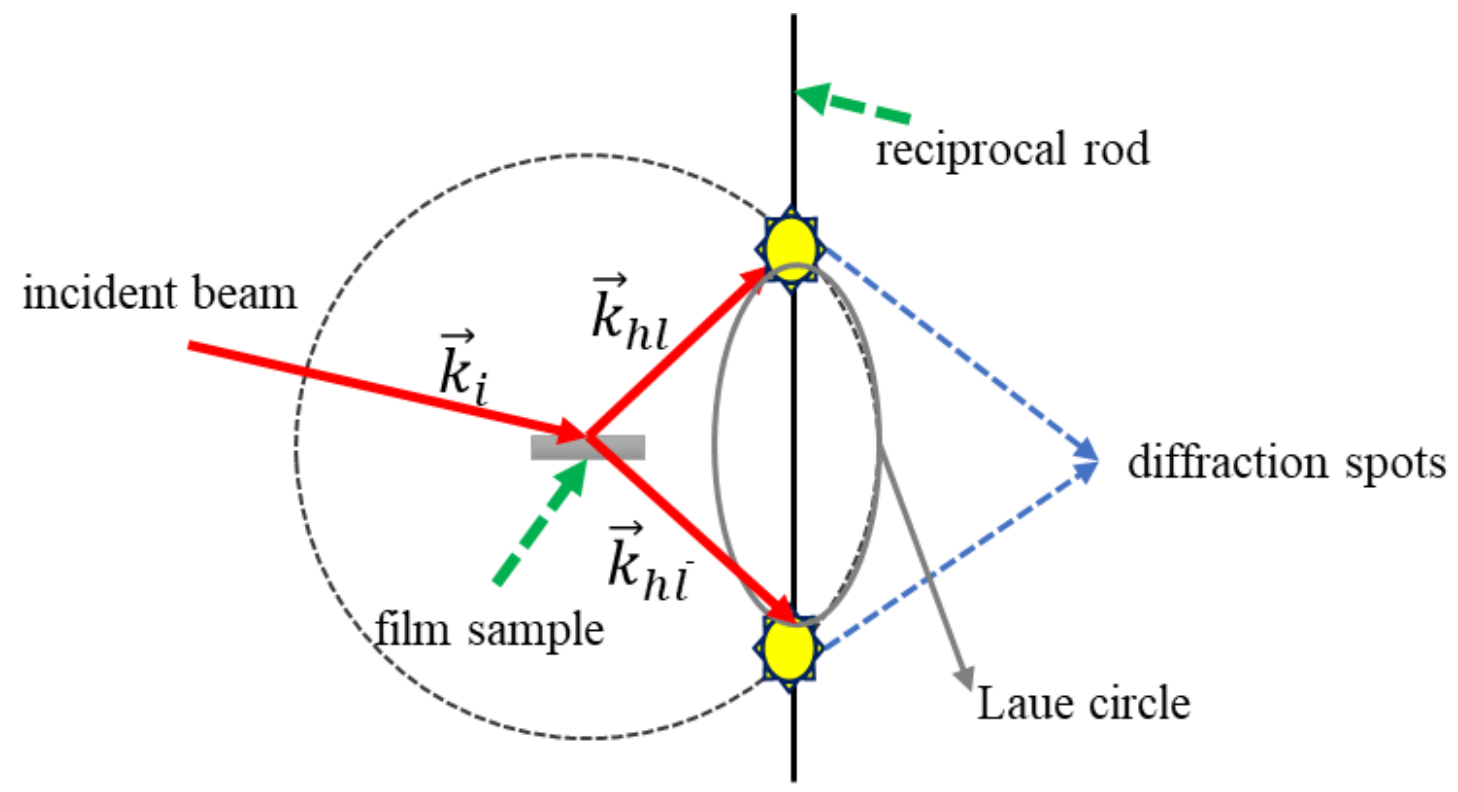

Figure 2.4 RHEED diffraction condition with the Ewald's sphere, Laue circle and the reciprocal rod presentation.

The RHEED pattern of the grown film is shown in Figure 2.4. Figure 2.5-a shows the RHEED pattern STO substrate. The intense specular spot compared with the two side spots is the typical signature of $\mathrm{TiO}_{2}$ terminated STO [18, 19]. Figure 2.5-b shows sharp 2-dimensional streaks lying on concentric Laue circles in the RHEED which confirms a smooth surface of the deposited thin film [9]. 

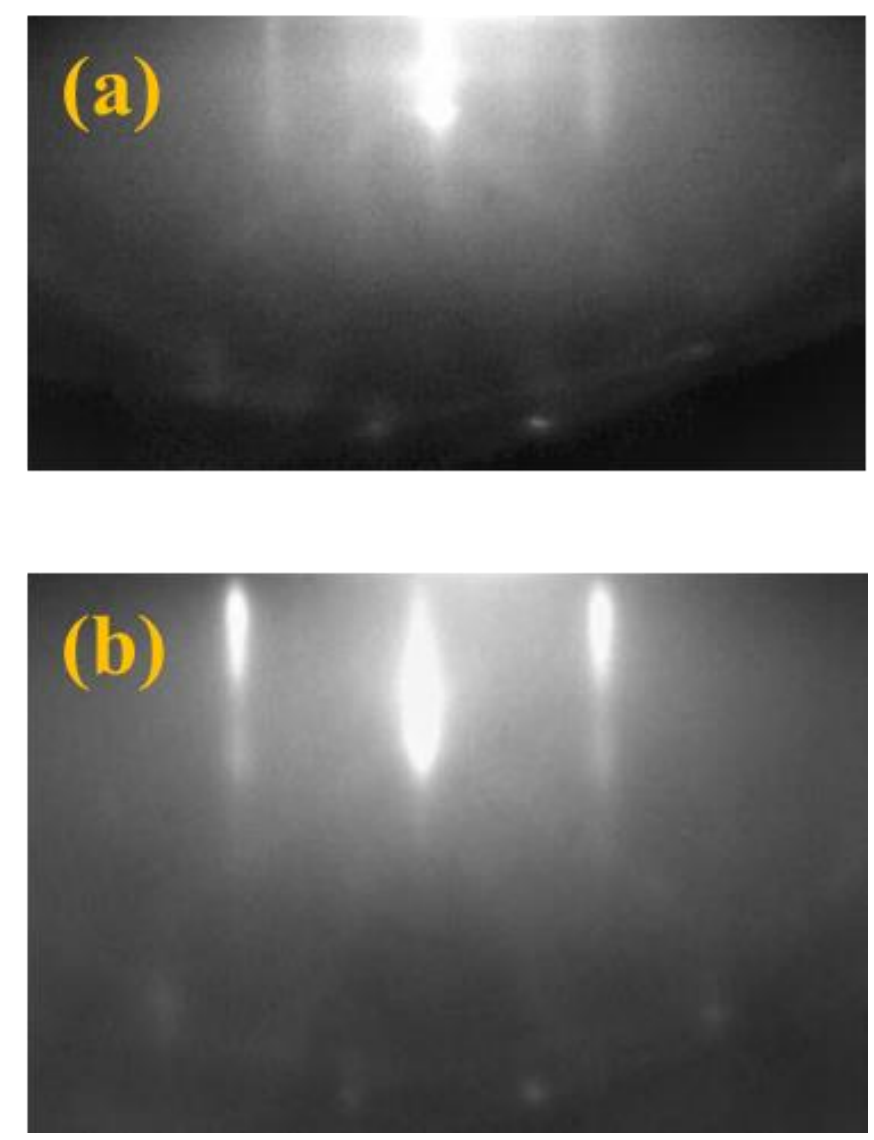

Figure 2.5. RHEED diffraction patterns of (a) STO substrate (b) $7.6 \mathrm{~nm} \mathrm{LSMO/STO}$ after growth.

\subsection{Structural Characterization}

The $\mathrm{x}$-ray diffraction (XRD) and $\mathrm{x}$-ray reflectivity (XRR) of samples was performed by using a Bruker D8 Discovery $x$-ray diffractometer on the samples to determine the crystallinity and the thickness of the film. The x-ray source was an anode $\mathrm{Cu} K_{\alpha}$ radiation with the wavelength, $\lambda=1.5405 \AA$.

\subsubsection{X-ray Diffraction}

As I discussed in section 1.3, LSMO film has a little lattice mismatch with STO which results in changing the crystal structure of the film and causing the tensile strain [10]. The tensile strain causes the peak shift of LSMO film. In order to clarify the effect of the tensile strain, I provided the XRD data of 7.6 $\mathrm{nm}$ LSMO/STO film along with a thicker sample of $32 \mathrm{~nm}$ LSMO/STO. The XRD data of the samples are shown in Figure 2.6. The LSMO peak is indexed using the pseudocubic structure with a single (001) orientation. and the LSMO peak appears on the right-hand side of the STO (001) peak in the $32 \mathrm{~nm}$ film (Figure 2.6-b). As the thickness of the sample decreases to $7.6 \mathrm{~nm}$, the LSMO (002) peak shifts toward STO (001) peak, and the LSMO peak appears as a broad shoulder beside the STO peak. 


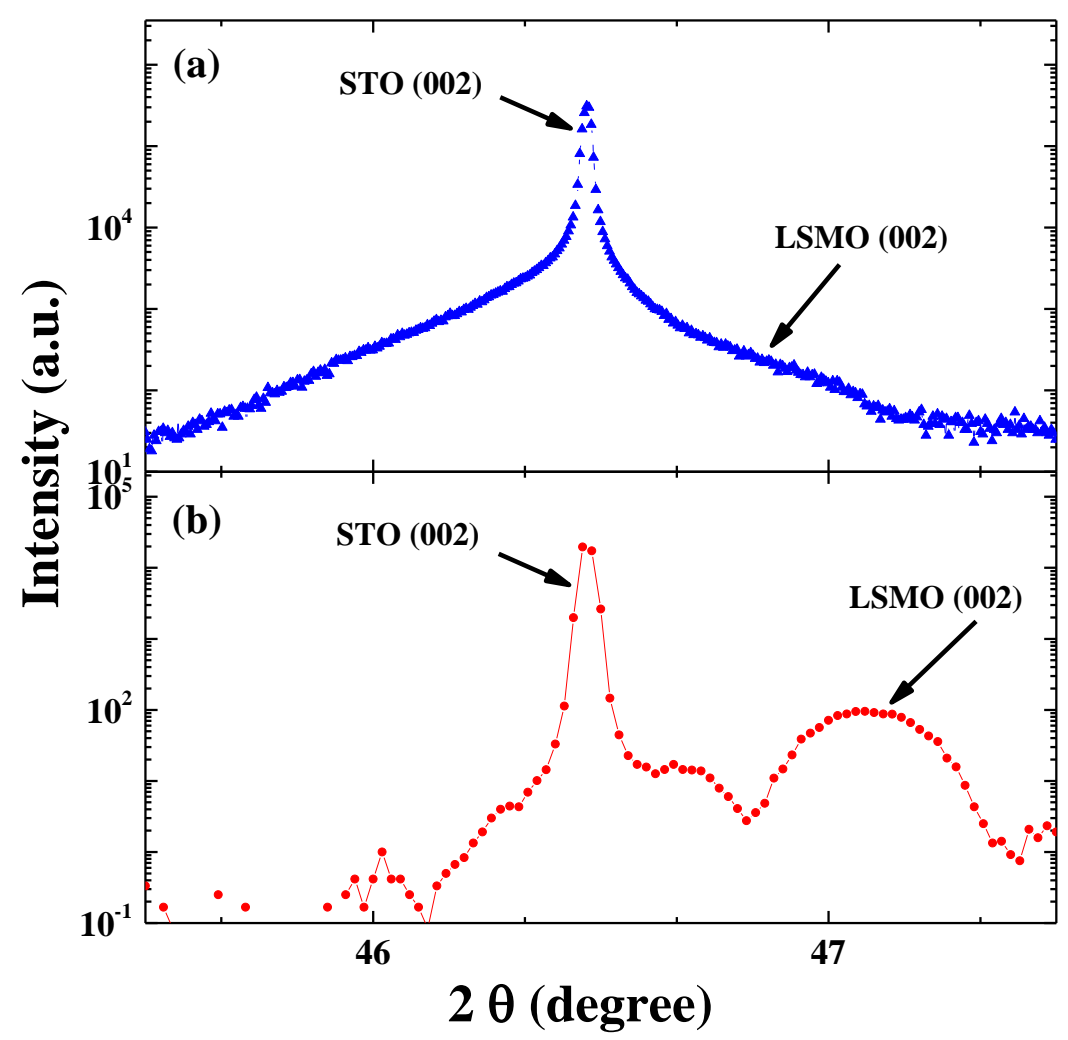

Figure 2.6. XRD scan of LSMO films (a) $7.6 \mathrm{~nm} \mathrm{LSMO/STO} \mathrm{and} \mathrm{(b)} 32 \mathrm{~nm} \mathrm{LSMO/STO} \mathrm{film.}$

\subsubsection{X-ray Reflectivity}

In XRR measurements, the X-ray beam is focused at a grazing angle on a flat surface of the sample. If the incident angle is below the critical angle for total reflection $\left(\theta_{\mathrm{C}}\right)$ then the incident $\mathrm{x}$-ray beam total reflection occurs. The interference between the reflected beams from the surface of the film and the interface between the film and the substrate creates oscillations [45]. These oscillations are called Kiessing fringes. If the interface has higher roughness, it can reduce the interference of the two beams. The rougher interface scatters the reflected beam from the interface, and it decrease the amplitude of oscillations therefore, interfaces with lower roughness have better interference. In XRR the amplitude and the period of oscillations depend on the physical properties of the film. The amplitude of oscillations can be affected by the density of the material, for example, materials with lower densities have a smaller amplitude of oscillations [45]. The period of the oscillations depends on the thickness of the film in which thicker films have a shorter period of oscillations [46]. 
The XRR measurement was performed using a Bruker D8 Discovery x-ray diffractometer on the sample. The x-ray source was an anode $\mathrm{Cu} K_{\alpha}$ and the beam was monochromatized using a $\mathrm{Ge}$ (111) monochromator. XRR was required to measure the thin film thickness which is a useful technique to measure microstructure parameters such as the thickness, density, and roughness of layers in thin films [47]. The experimental and simulated XRR curves for $7.6 \mathrm{~nm} \mathrm{LSMO/STO} \mathrm{thin} \mathrm{film} \mathrm{are} \mathrm{shown} \mathrm{in} \mathrm{Figure}$ 2.7. as common for other high-quality LSMO films, the two layers were required to fit the XRR data well. A two-layer model has been previously used in LSMO thin films [48]. The two slab model and multi-layer models have also been used in other systems when the density of the material changes [43, 44]. The simulated XRR result shows the presence of $7.6 \mathrm{~nm} \mathrm{LSMO/STO} \mathrm{film} \mathrm{with} \mathrm{the} \mathrm{two-layer} \mathrm{model} \mathrm{fit.} \mathrm{Each}$ layer has a roughness of $0.52 \mathrm{~nm}$.

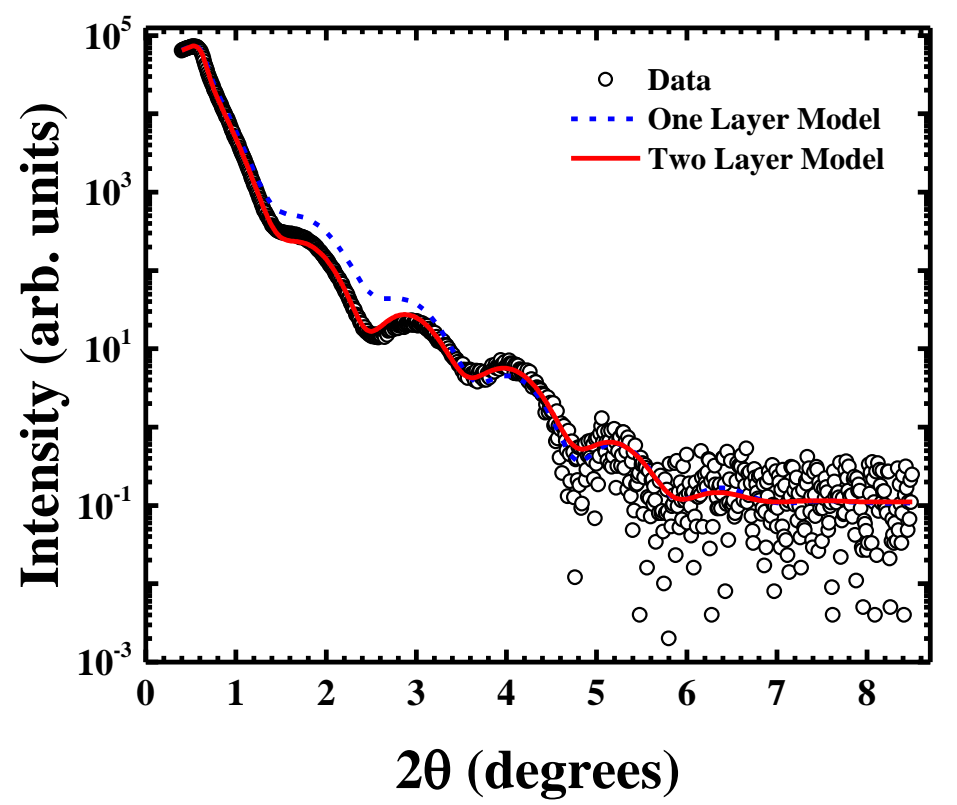

Figure 2.7. XRR profile for the $7.6 \mathrm{~nm}$ LSMO/STO thin film sample. The blue dashed line and the continuous red line are the fits to one-layer and the two-layer models respectively.

\subsection{Summary}

In this chapter, I discussed the substrate treatment and the PLD fabrication process of a typical LSMO film and also the structural characterizations such as XRR and XRD. In the next chapter, I discuss the theoretical background and dc magnetic measurements. 


\section{Chapter 3 \\ Investigation of the Magnetic Dead Layer Properties by DC- Magnetometry Measurements}

\subsection{Introduction}

In this chapter, I discuss the theoretical approaches which are used to analyze the ac and dc magnetic susceptibility data presented in this dissertation. I study the magnetic properties of a well-known complex oxide system, $\mathrm{La}_{1-\mathrm{x}} \mathrm{Sr}_{\mathrm{x}} \mathrm{MnO}_{3}$ with the optimum ratio of $\mathrm{x} \sim 0.3$ which is famous for the loss of magnetization and having the magnetic dead regions. I explore the loss of saturation magnetization by employing molecular mean-field and spin-wave theories to understand the temperature variation of magnetization. The goal of this dissertation is to understand the hidden magnetic properties of MDLs. Some similarities are found in the magnetic properties of LSMO films compared with nanoparticles where LSMO thin films show magnetic inhomogeneities (MI). Therefore, I use famous theories on single-domain nanoparticle systems to describe the reduction of temperature variations of magnetization.

The theories discussed here are gathered from textbooks by Kittel (Introduction to solid-state physics. Wiley, 2005), A. H. Morrish (The Physical Principles of Magnetism. Wiley-IEEE PRESS), N. A. Spaldin (Magnetic materials: fundamentals and applications. Cambridge university press, 2010) and S. Blundell

("Magnetism in condensed matter." American Association of Physics Teachers, 2003). The experimental results of this chapter have been published by the author recently in scientific journals (N. Mottaghi, et al, J. Appl. Phys. 128, 073903 (2020); N. Mottaghi, et al, J. Phys. Condens. Matter 30, 405804 (2018); and N. Mottaghi, et al, AIP Adv. 8, 56319 (2018)) and presented at MMM 2017 - 62nd Annual Conference on Magnetism and Magnetic Materials, American Vacuum Society (AVS 65th International Symposium \& Exhibition), and 64h Annual Conference on Magnetism and Magnetic Materials.

\subsection{Theoretical background}

In this theoretical section, first, I discuss the molecular mean-field theory then I explain the spin-wave theory. Both theories are used to analyze the temperature variation of saturation magnetization in LSMO films. I explain the SPM magnetic characteristics and in section 3.2.4, and I go through the total energy of mixed magnetic states in which two magnetic phases co-exist in a single magnetic sample.

\subsubsection{Molecular Field Theory and Spin Waves}

In section 1.2, magnetization is explained as the sum of an infinite number of currents passing through the closed loops which is the simple and classical picture presentation of magnetization, but in this section, I want to discuss the concept of magnetization in the quantum mechanical picture. 
The magnetic moment of a magnetic material depends on the atomic angular momentum quantum number $(J)[4,51]$

$$
M=-\frac{g \mu_{B}}{\hbar} J
$$

which $\mu_{B}$ is the Bohr magneton, and $J$ is the sum of spin and orbital angular momentum, which has the range of values of

$$
J=(L-S),(L-S+1), \ldots,(L+S+1),(L+S),
$$

where $S$ and $L$ are the total spins and total orbital angular momentum, respectively; and $g$ is the Landé $g$ factor which is

$$
g=1+\frac{J(J+1)+S(S+1)-L(L+1)}{2 J(J+1)}
$$

The Hamiltonian of the magnetic material in the external magnetic field is $H=-M . B_{\text {ext }}$, where $B_{\text {ext }}$ is the external magnetic field. The energy of the system is the expectation value of the Hamiltonian thus [51]

$$
E_{j s}=<j s\left|-M \cdot B_{\text {ext }}\right| j s>=\frac{g \mu_{B}}{\hbar}<j s\left|J \cdot B_{\text {ext }}\right| j s>.
$$

According to statistical mechanics, the energy of the system is determined by the Boltzmann factor $\exp \left(-E_{j s} / k_{B} T\right)$ then we have

$$
M=-\frac{1}{V} \frac{\sum_{-J}^{J} M g \mu_{B} e^{-\frac{g \mu_{B} M B_{e x t}}{k_{B} T}}}{\sum_{-J}^{J} e^{-\frac{g \mu_{B} M B_{e x t}}{k_{B} T}}},
$$

which $M=N g \mu_{B} J B_{J}(y)$, and $B_{J}(y)$ is called as Brillouin function [51]

$$
B_{J}(y)=\frac{2 J+1}{2 J} \operatorname{coth}\left(\frac{2 J+1}{2 J} y\right)-\frac{1}{2 J} \operatorname{coth}\left(\frac{1}{2 J} y\right), \quad y=g \mu_{B} J B_{\text {ext }} /\left(k_{B} T\right) .
$$

In the limit of high temperature or high external magnetic field $(y \ll 1)$ the Brillouin function is approximately [4]

$$
B_{J}(y)=\frac{y}{3}\left\{\frac{(2 J+1)^{2}-1}{(2 J)^{2}}\right\}
$$


and magnetization becomes $M=\left[N g^{2} \mu_{B}^{2} J(J+1) / 3 k_{B} T\right] B_{\text {ext }}$ which the magnetic susceptibility is

$$
x_{0}=\frac{\partial M}{\partial H}=\frac{C}{T}
$$

where

$$
C=\frac{N g^{2} \mu_{B}^{2} J(J+1)}{3 k_{B}}
$$

which is called the Curie constant. Equation-(3-8) is the Curie law which presents the temperature variation of $x_{0}$ but we did not include the effect of magnetic ions on each other.

The FM material has the $M_{\mathrm{SP}}$ without exposure to an external magnetic field. In 1907 Pierre Weiss proposed a model to explain magnetism in magnetic materials [52]. He proposed an internal field that mainly comes from the magnetic ions of the material. This field is $H_{\mathrm{m}}=N_{\mathrm{w}} M$ which $N_{\mathrm{W}}$ is the molecular field constant. The total field $\left(H_{T}\right)$ on a magnetic ion is [4]

$$
H_{T}=B_{\text {ext }}+N_{W} M
$$

By calculating the expectation value of Hamiltonian by replacing the $B_{\text {ext }}$ by $H_{T}$ in the equation-(3-4) the $y$ component becomes $\left(g \mu_{B} J\left(B_{\text {ext }}+N_{W} M\right)\right) /\left(k_{B} T\right)$ and in the limit of $y \ll 1$, magnetization is

$$
M(T)=\frac{C}{T}\left(B_{\text {ext }}+N_{W} M\right)
$$

which $C$ is the Curie constant which is defined above.

The magnetic susceptibility in the presence of the molecular field is [51]

$$
x=\frac{\partial M}{\partial H}=\frac{C}{T-\theta},
$$

where $\theta=C N_{W}=T_{\mathrm{C}}$. Equation-(3-12) is the Curie-Weiss law [4,51]. The sign of $\theta$ parameter is important which in FM and AFM materials is positive and negative, respectively and in the case of $\theta=0$, equation(3-12) changes into the equation-(3-8).

Another useful model to describe the temperature variation of $M_{\mathrm{SP}}$ is the spin-wave theory. This theory is based on the Heisenberg exchange interaction Hamiltonian [53]. In this theory, at $T=0 \mathrm{~K}$, all the spins of the magnetic system are well-ordered which satisfies the magnetic ground state of the system. As the temperature is increased such that one spin is flipped then the system goes into the lowest excited state. The 
flipped spin does not remain to localize at one atom, and each magnetic atom has an equal probability of having spin reversed. The propagation of the reversed spin through the crystal is called a spin-wave [4].

The Hamiltonian of the system considering only the exchange interaction between the nearest neighbor spins $[51,54]$ is

$$
H_{e x c}=-2 \sum_{i=1}^{N} J_{a b} S_{i} S_{i+1},
$$

where $S_{\mathrm{i}}$ is the spin on atom " $i$ ", $S_{\mathrm{i}+1}$ is the spin on atom " $i+1$ " which is the nearest neighbor concerning atom " $i$ " and $J_{a b}$ is the exchange integral. The $J_{a b}$ depends on the wave functions of electrons 1 and 2 at sites $i$ and $i+1$ which is given by [4]

$$
J_{a b}=\int \psi_{a}^{*}\left(r_{1}\right) \psi_{b}^{*}\left(r_{2}\right) \frac{e^{2}}{r_{12}} \psi_{b}\left(r_{1}\right) \psi_{a}\left(r_{2}\right) d^{3} r_{1} d^{3} r_{2}
$$

and depends on the overlap of the wave space functions, and the Pauli repulsion [50, 48].

One can find the exchange field on one spin " $p$ " simply by writing the Hamiltonian for that spin by using equation-(3-13), and $J_{a b}=J_{e}$ thus

$$
H_{\text {exc }}=-2 J_{e} S_{P} \cdot\left(S_{P+1}+S_{P-1}\right)
$$

where $S_{p}$ is the spin of atom " $p$ ".

Equation(3-15) can be simplified by substituting $S_{p}=-\mu_{p} / g \mu_{B} \cdot g$ is a called the $g$ factor which is defined in the equation-(3-3). Equation-(3-15) changes into

$$
H_{e x c}=-\mu_{p} \cdot B_{p}
$$

where $B_{p}=\left(-2 J_{e} / g \mu_{B}\right) \cdot\left(S_{P+1}+S_{P-1}\right) . B_{p}$ is called the effective magnetic field or exchange field which applies on $p$ th spin. $B_{p}$ causes a torque on the $p$ th spin, that we can calculate the rate change of the $S_{p}$ by [51]

$$
d S_{p} / d t=-\left(g \mu_{B} / \hbar\right) S_{p} \times B_{p}=\left(2 J_{e} / \hbar\right)\left(S_{p} \times S_{p-1}+S_{p} \times S_{p+1}\right) .
$$

In equation-(3-17), $S_{p}$ is a vector with three components $S_{p}^{x}, S_{p}^{y}$ and $S_{p}^{z}$. We can solve equation-(3-17) in the Cartesian coordinate system thus (3-17) breaks down into three equations, 


$$
\begin{aligned}
& d S_{p}^{x} / d t=\left(2 J_{e} / \hbar\right)\left[S_{p}^{y}\left(S_{p-1}^{z}+S_{p+1}^{z}\right)-S_{p}^{z}\left(S_{p-1}^{y}+S_{p+1}^{y}\right)\right] \\
& d S_{p}^{y} / d t=-\left(2 J_{e} / \hbar\right)\left[S_{p}^{x}\left(S_{p-1}^{z}+S_{p+1}^{z}\right)-S_{p}^{z}\left(S_{p-1}^{x}+S_{p+1}^{x}\right)\right] \\
& d S_{p}^{z} / d t=\left(2 J_{e} / \hbar\right)\left[S_{p}^{x}\left(S_{p-1}^{y}+S_{p+1}^{y}\right)-S_{p}^{y}\left(S_{p-1}^{x}+S_{p+1}^{x}\right)\right] .
\end{aligned}
$$

If we assume the $S_{p}^{x}$ and $S_{p}^{y}$ are small in comparison with the amplitude of excitation, then $S=S_{p}^{z}$ and equations-(3-18)

$$
\begin{aligned}
& d S_{p}^{x} / d t=\left(2 J_{e} S / \hbar\right)\left[2 S_{p}^{y}-S_{p-1}^{y}-S_{p+1}^{y}\right] \\
& d S_{p}^{y} / d t=-\left(2 J_{e} S / \hbar\right)\left[2 S_{p}^{x}-S_{p-1}^{x}-S_{p+1}^{x}\right] \\
& d S_{p}^{z} / d t=0 .
\end{aligned}
$$

The solutions of the equation-(3-19)

$$
\begin{aligned}
& S_{p}^{x}=u \exp [i(p k a-\omega t)], \\
& S_{p}^{y}=v \exp [i(p k a-\omega t)],
\end{aligned}
$$

where $u, v$ are constants, $p$ is an integer, $a$ is the lattice constant and $\omega$ is the frequency of spin waves.

By substituting equations-(3-19) into the equation-(3-20) then

$$
\begin{aligned}
& -i \omega u=\left(2 J_{e} S / \hbar\right)(2-\exp [-i k a]-\exp [i k a]) v=\left(4 J_{e} S / \hbar\right)(1-\cos (k a)) v \\
& -i \omega v=-\left(2 J_{e} S / \hbar\right)(2-\exp [-i k a]-\exp [i k a]) u=-\left(4 J_{e} S / \hbar\right)(1-\cos (k a)) u
\end{aligned}
$$

To solve equations-(3-21), we need to set the determinant of coefficients $u$ and $v$ to zero [51]:

$$
\left|\begin{array}{cc}
i \omega & \left(4 J_{e} S / \hbar\right)(1-\cos (k a)) \\
-\left(4 J_{e} S / \hbar\right)(1-\cos (k a)) & i \omega
\end{array}\right|=0
$$

which gives us the dispersion relation for $\omega$,

$$
\hbar \omega=\left(4 J_{e} S\right)(1-\cos (k a)) .
$$

Equation-(3-23) leads to an important relation between the $\omega$, and $J_{e}$, spin and the lattice constant. For long wavelength, $k(=2 \pi / \lambda)<<1$, equation-(3-23) becomes $\hbar \omega \approx 2 J_{e} S k^{2} a^{2}$ which eventually yields the Bloch law. The energy of spin waves is the same as a harmonic oscillator of the same frequency thus the energy of frequency $\omega_{k}$ with $k$ th oscillation mode $\left(n_{k}\right)$ is given by 
$\epsilon_{k}=\left(n_{k}+1 / 2\right) \hbar \omega_{k}$

Spin waves are called magnons as well. The total number of magnons can be calculated by the Plank distribution

$$
<n_{k}>=1 /\left(\exp \left(\hbar \omega_{k} / k_{B} T\right)-1\right)
$$

The total number of excited magnons at a temperature $T$ is given by

$$
\sum_{k} n_{k}=\int d \omega D(\omega)<n(\omega)>
$$

In equation-(3-26), $D(\omega)$ is the number of magnon modes per unit frequency range which is also called the density of modes of magnons. The integral is all over the range of $k$ in the first Brillion zone. In threedimensional space, the number of modes of wavevectors is $(1 / 2 \pi)^{3}\left(4 \pi k^{3} / 3\right)$. The number of magnons $D(\omega) d \omega$ with frequency in $\mathrm{d} \omega$ at $\omega$ is $(1 / 2 \pi)^{3}\left(4 \pi k^{2}\right)(d k / d \omega) d \omega$. From equation-(3-23) in approximation $k<1$ and taking the derivative respect to $k$ thus

$$
d \omega / d k=4 J_{e} S a^{2} k / \hbar
$$

since $k \approx a\left(\hbar \omega / 2 J_{e} S\right)^{1 / 2}$ and equation-(3-27) becomes

$$
d \omega / d k=2\left(2 J_{e} S a^{2} / \hbar\right)^{1 / 2} \omega^{1 / 2}
$$

We can calculate $D(\omega)$ by inserting it into $(1 / 2 \pi)^{3}\left(4 \pi k^{2}\right)(d k / d \omega) d \omega$ equation which yields

$$
D(\omega)=1 / 4 \pi^{2}\left(\hbar / 2 J_{e} S a^{2}\right)^{3 / 2} \omega^{1 / 2}
$$

By inserting the equation-(3-29) into the equation-(3-26) and using the equation-(3-25) the total number of magnons is

$$
\sum_{k} n_{k}=\left(1 / 4 \pi^{2}\right)\left(\hbar / 2 J_{e} S a^{2}\right)^{\frac{3}{2}} \int_{0}^{\infty} d \omega \frac{\omega^{\frac{1}{2}}}{\exp \left(\beta \hbar \omega_{k}\right)-1}
$$

In equation-(3-30), the integral has the value $(0.0587)\left(4 \pi^{2}\right)$. The fractional change of magnetization $\Delta M / M(0)$ is equal to the ratio of the total number of magnons for the total number of spins which is 


$$
\sum_{k} n_{k} / N S=\Delta M / M(0)
$$

where $N$ is the number of atoms which the magnetic atoms per unit volume $\mathrm{Q} / a^{3}$. Equation-(3-31) turns into

$$
\Delta M / M(0)=(0.0587 / \mathrm{SQ}) \cdot\left(k_{B} T / 2 J_{e} S\right)^{3 / 2} .
$$

Equation-(3-32) is the Bloch $T^{3 / 2}$ law equation [4,51]. The concept of the Bloch law is used in this chapter for the interpretation of the temperature dependence of the saturation magnetization.

\subsection{What is the Relation Between the Molecular Field and the Exchange Interaction?}

If all the spins have the same $J_{e}$ in the equation-(3-13) with the $z$ nearest neighbors then the exchange energy is the sum over all of the nearest neighbors [4]

$$
<H_{e x c}>=-2 J_{e}<S_{i}>. \sum_{i=1}^{Z} S_{i+1} .
$$

The sum of all nearest neighbors $\sum_{i=1}^{Z} S_{i+1}$ produces the field which is

$$
H_{m}=\frac{2 J_{e} \sum_{i=1}^{Z} S_{i+1}}{g \mu_{B}}=\frac{2 Z J_{e}<S_{i+1}>}{g \mu_{B}} .
$$

From the molecular field theory equation-(3-10) the total field in the absence of an external $B_{\text {ext }}$ field is $H_{\mathrm{T}}=N_{\mathrm{W}} M$. Since the molecular field and exchange field are produced from the nearest neighbors, they are equivalent, therefore [51]

$$
N_{W} M=\frac{2 Z J_{e}<S_{i+1}>}{g \mu_{B}}
$$

assuming the spin of all magnetic neighbors are the same $\left\langle S_{i+1}\right\rangle=\langle S\rangle$ and the value of magnetization $M=N<S>$ which by substituting these values in equation-(3-35), we get a valuable relation between the molecular field constant and the exchange constant,

$$
N_{W}=\frac{2 Z J_{e}}{N g^{2} \mu_{B}^{2}}
$$

As we know the $T_{C}=C N_{\mathrm{w}}$, then there is a valuable relation between the $T_{C}$ and $J_{e}$ [51]

$$
T_{C}=\frac{2 Z J_{e} J(J+1)}{3 k_{B}}
$$


Equation-(3-37) is used to calculate the $J_{e}$ value based on the value of $T_{C}$ obtained from the inflexion point of the temperature variation of magnetization in section 3.4.1. Also, the calculated value of $J_{e}$ of this equation is compared with result of Bloch law (equation-(3-32)) in analyzing the temperature variation of saturation magnetization.

\subsubsection{Superparamagnetism}

I briefly discussed the SPM phase in section 1.2.3.2 and here I discuss more the magnetic characteristics of this magnetic phase. The magnetic characterization results of the $7.6 \mathrm{~nm} \mathrm{LSMO/STO}$ sample reveal similar characteristics of the SPM phase such as the blocking temperature, hysteresis loop inversions, and magnetic viscosity relaxations. Thus, important magnetic characteristics of this phase are covered here.

\subsubsection{Blocking Temperature, Energy Barrier, and Magnetic Viscosity}

The energy of the particle is described as $\operatorname{KVSin}^{2}(\theta)$ where $\theta$ is the angle between the $M$ and the monocrystalline anisotropy, $V$ is the volume of the particle, and $K$ is the uniaxial anisotropy constant. There are two local minima in this energy system, one at $\theta=0$ and the other at $\theta=\pi$ [56]. The energy difference between the two local minima is the energy barrier of the system: $\Delta E=K V$ which depends on the $V$.

For small particles, the energy barrier height becomes small and comparable with the thermal energy, $k_{\mathrm{B}} T$, in which $k_{\mathrm{B}}$ is the Boltzman constant and $T$ is the temperature. At this temperature which is called blocking temperature $\left(T_{\mathrm{B}}\right)$, the thermal energy can switch the magnetic moment of the particles from $\theta=0$ to $\theta=\pi$ and vice versa such that the particle behaves like a PM. The energy barrier can be changed if the particle is exposed to a magnetic field $(H)$ then the energy of the particle changes into

$$
E=K V \operatorname{Sin}^{2}(\theta)-H \mu \operatorname{Cos}(\theta)
$$

To find the local minimum of the system in the applied field, it can be found by taking the derivative of $E$ with respect to $\theta$. Thus

$$
\frac{\partial E}{\partial \theta}=2 K V \operatorname{Sin}(\theta) \operatorname{Cos}(\theta)+H \mu \operatorname{Sin}(\theta)=0
$$

which yields three extremums: $\theta=0, \pi, \operatorname{Cos}^{-1}\left(-\frac{H \mu}{2 K V}\right)$. The values of $E$ for these extremums are: $\theta=0$, $E=-\mu H ; \theta=\pi, E_{\text {antiparallel }}=+\mu H ;$ and $\theta=\operatorname{Cos}^{-1}(-H \mu /(2 K V)), E_{\max }=K V\left[1+\left(\frac{H \mu}{2 K V}\right)^{2}\right]$. 
The energy difference between the antiparallel energy $\left(\theta=\pi, E_{\text {antiparallel }}=H M\right)$ and maximum value of energy $\left(\operatorname{Cos}^{-1}\left(-\frac{H \mu}{2 K V}\right), E_{\max }=K V\left[1+\left(\frac{H \mu}{2 K V}\right)^{2}\right]\right)$ is the energy barrier that a magnetic moment needs to align in the direction of $H$.

Thus, the energy barrier in the presence of $H$ is

$$
\Delta E=E_{\text {max }}-E_{\text {antiparallel }}=K V\left[1+\left(\frac{H \mu}{2 K V}\right)^{2}\right]-M H=K V\left(1-\frac{H \mu}{2 K V}\right)^{2}
$$

where $H_{k}=2 K V / \mu$, the $\Delta E=0$. The $H_{k}$ is called an anisotropy field and $\mu$ is the saturation magnetization where $\mu / V$ is the magnetization per unit volume $(M)$.

The time variation of a magnetic system that reaches a stable state can be written as $\mathrm{d} M(t) / \mathrm{d} t=-(M(t)-M(t=\infty)) / \tau$ which the $M(t=\infty))$ is the stable state of the magnetization and $\tau$ is a characteristic relaxation time corresponding to a single energy barrier for a uniaxial anisotropic system can be given by the Arrhenius Néel law [57,58]

$$
\tau^{-1}=f=f_{0} e^{-\Delta E /\left(k_{B} T\right)}
$$

where $\Delta E$, as defined in the equation-(3-40), is the energy barrier. $f_{0}$ is the characteristic frequency of the system. In equation-(3-41), $f$ relaxation depends on the $H$, the volume of the particle, and the anisotropy constant $[59,60]$.

If we use the energy barrier of the system from the equation-(3-41) and insert it in the equation-(3-40), we can obtain a relation between the $f_{0}$ and the anisotropy field,

$$
\ln \left(\tau f_{0}\right) k_{\mathrm{B}} T=K V\left(1-\frac{H}{H_{k}}\right)^{2}
$$

To simplify the equation-(3-42), we need to divide both sides of the equation by $K V$ then equation(3-42) becomes

$$
H=H_{k}\left(1-\left(\frac{k_{\mathrm{B}} T}{K V} \ln \left(\tau f_{0}\right)\right)^{1 / 2}\right)
$$

which simply shows the relation between the required field to switch the magnetization direction and the $f_{0}$. The required field $(H)$ to switch the magnetization direction over the energy barrier is often noted as $H_{\mathrm{C}}$ that equation-(3-43) presents the frequency and temperature dependence of the coercive field [61]. The $H$ $\left(=H_{\mathrm{C}}\right)$ value can be zero at a certain frequency and a relaxation time: 


$$
k_{\mathrm{B}} T_{B} \ln \left(\tau_{0} f_{0}\right)=K V
$$

which occurs at the blocking temperature.

For an arbitrary measurement of $\tau_{0}=100 \mathrm{~s}$ and $f_{0}=10^{9} \mathrm{~s}^{-1}, K V \sim 25 k_{\mathrm{B}} T_{B}$. This energy is the critical energy barrier ( $\Delta E_{\text {critical }}$ ) where thermal fluctuations can destroy the stable state of magnetization. In 1949 , Louis Néel proved that for small particles at $T=T_{B}$ with $\Delta E_{\text {critical }}$, the magnetic moments are randomly oriented and the remanent magnetization is zero and subsequently $H_{\mathrm{C}}=0$ [62], which is the signature of the SPM phase.

In equation-(3-41), at certain temperatures $T=T_{\mathrm{B}}$ the $f$ matches the measuring frequency which leads to

$$
T_{B}=\Delta E /\left[\left(k_{B} \ln \left(f_{0} / f_{m}\right)\right]\right.
$$

which is valid for magnetic particles that do not interact with each other [63].

In the case of interparticle interaction the term $T_{0}$ is added to the model which presents the strength of the interaction

$$
T_{B}=T_{0}+\Delta E /\left[\left(k_{B} \ln \left(f_{0} / f_{m}\right)\right]\right.
$$

and by simplifying the equation to express the relaxation time in terms of $T_{0}$ leads into Vogel-Fulcher law [64]

$$
\tau=\tau_{0} e^{\Delta E /\left(k_{B}\left(T-T_{0}\right)\right)} .
$$

To obtain a relation between $H_{\mathrm{C}}$ and $T_{\mathrm{B}}$ in equation-(3-43) and equation-(3-44), we can substitute $K V / k_{\mathrm{B}} \ln \left(\tau_{0} f_{0}\right)$ with $T_{\mathrm{B}}$ from equation-(3-44), thus we have the new form of the equation-(3-43)

$$
H_{\mathrm{C}}=H_{k}\left(1-\left(\frac{T}{T_{B}}\right)^{1 / 2}\right)
$$

which presents the relationship between $T_{\mathrm{B}}$ and $H_{\mathrm{C}}[19,54,57]$. If we rewrite this equation to express the functionality of $T_{\mathrm{B}}$ with respect to $H_{\mathrm{C}}$ then equation-(3-48) changes into

$$
T=T_{\mathrm{B}}\left(1-\frac{H}{H_{\mathrm{C}}}\right)^{2}
$$

the equations-(3-48) and (3-49) are used in data analysis in this dissertation. 
In reality all magnetic components of a magnetic system have a wide distribution of energy barriers and a simple solution to $\mathrm{d} M(t) / \mathrm{d} t=-(M(t)-M(t=\infty)) / \tau$ is to consider only single relaxation time. Thus, the magnetization of the system can be written as the distribution of energy barrier as

$$
M(t)=\int_{0}^{\infty} d E f(E) e^{-t / \tau(E)},
$$

where $f(E)$ is the distribution of energy barriers and $\tau$ defined in the equation-(3-41). Equation-(3-50) can be written in terms of $\tau$ which is achievable simply by taking the derivative respect to $E$ in the equation$(3-41)$

$$
d \tau=\left(\left(\tau_{0} e^{\frac{\Delta E}{k_{B} T}}\right) / k_{B} T\right) d E,
$$

which $\tau_{0}=1 / f_{0}$, thus $d E$ in terms of $d \tau$ is

$$
d E=\frac{k_{B} T d \tau e^{-\frac{\Delta E}{k_{B} T}}}{\tau_{0}}
$$

By inserting equation-(3-52) into the equation-(3-50)

$$
M(t)=\int_{0}^{\infty} \frac{k_{B} T f(E) e^{-\frac{t}{\tau(E)}}}{\tau(E)} d \tau,
$$

where $\tau(E)=\tau_{0} e^{\frac{\Delta E}{k_{B} T}}$. Equation-(3-53) presents the time variation of magnetization of a magnetic system over the distribution of energy barriers. To find the time variation of magnetization, we can take the derivative with respect to time thus we have

$$
d M(t) / d t=\int_{0}^{\infty}-\frac{k_{B} T f(E) e^{-\frac{t}{\tau(E)}}}{\tau(E)^{2}} d \tau,
$$

assuming $f(E)=E_{0}$ then the solution of the integral in the equation-(3-54) is

$$
\frac{d M(t)}{d t}=-k_{B} T E_{0}\left(\frac{1-e^{-\frac{t}{\tau_{0}}}}{t}\right)
$$

Equation-(3-55) presents the time derivative of magnetization. To solve this equation here we consider the long relaxation time as $t \gg \tau_{0}$ then the term $e^{-\frac{t}{\tau_{0}}} \sim 0$ and the time variation of magnetization in this time limit is achievable by taking the integral respect with time which the final result is 


$$
M(t)=M_{0}-S \ln \left(\frac{t}{\tau_{0}}\right)
$$

where $S\left(=k_{B} T E_{0}\right)$ is called magnetic viscosity. The logarithmic decay of magnetization is observed in LSMO film and equation-(3-56) is used in the analysis of $M(t)$. The viscosity measurements are done by cooling the magnetic sample in the presence of a DC applied field $\left(H_{\mathrm{dc}}\right)$ to a measured temperature, and after the temperature is stabilized, the time variation of magnetization is recorded in no applied field.

The magnetic system with wide energy distribution has temperature-dependent behavior of magnetic viscosity $(S v s . T)$ and it has a maximum at the $T_{\mathrm{B}}$ (equation-(3-45)). In the case of interparticle interaction, the $T_{\mathrm{B}}$ follows the equation-(3-46) which is the function of the measured frequency, therefore, ac magnetic susceptibility measurements at $T_{\mathrm{B}}$ are required to understand the dynamic effects of the system which is discussed in chapter 5. Next, I discuss the experimental results of dc magnetometry measurements.

\subsection{Experimental Results and Discussions: DC Magnetometry Measurements and Modeling}

In this section, DC magnetic measurements of a $7.6 \mathrm{~nm} \mathrm{LSMO/STO}$ film and the results and analysis of these measurements are discussed in detail. The field and temperature variation of magnetization of the sample were measured by using a vibrating sample magnetometer (VSM) with a Quantum Design physical property measurement system (PPMS-9T).

\subsubsection{Investigation of Saturation Magnetization}

The temperature dependence of saturation magnetization $\left(M_{\mathrm{S}}\right)$ was measured by performing zero-fieldcooled (ZFC) hysteresis loops over a temperature range from $5 \mathrm{~K}$ to $400 \mathrm{~K}$. The coils were first demagnetized at room temperature then the sample was cooled to the measured temperature. The data were corrected for the diamagnetic contribution of the substrate which was evident from the negative slope of the $M$ vs $H$ plots at higher $H$ [3]. This linear diamagnetic susceptibility was used to correct the measured isothermal magnetization of the sample at each $H$ by subtracting the negative slope from the hysteresis loop data. The hysteresis loop is shown here is an example correcting for the background diamagnetic contribution of the substrate. The figure given below shows the measured hysteresis loop at $5 \mathrm{~K}$ for the ZFC case clearly showing the diamagnetic susceptibility at higher $H$ and the corrected loop after the diamagnetic contribution of the substrate is taken into account. The loop shown in Figure 3.1 is only up to $4 \mathrm{kOe}$ since the magnetization is well saturated at $4 \mathrm{kOe}$. 


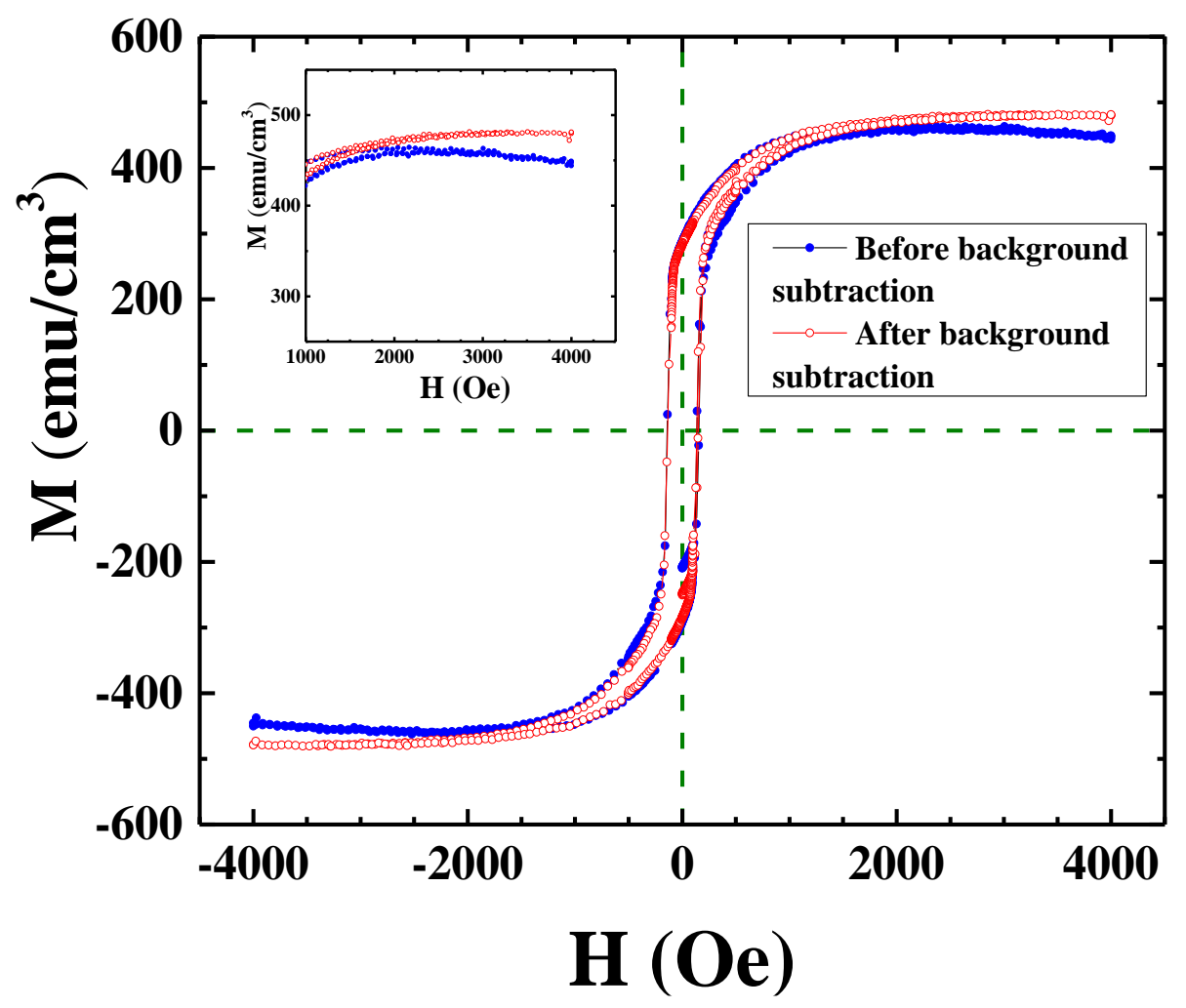

Figure 3.1. The ZFC hysteresis loop was measured at $5 \mathrm{~K}$ before and after background subtraction.

The temperature variation of $M_{\mathrm{S}}$ values was measured from the all ZFC hysteresis loop at $H=4 \mathrm{kOe}$ and plotted in Figure 3.2. The $M_{\mathrm{S}}$ vs. $T$ data is fit to the equation-(3-32) (Bloch law) to obtain the $J_{e}$. As I discussed in section 1.3.1, in manganates, $\mathrm{Mn}$ ion is the only magnetic ion contributing to magnetization thus $Q=1$ and $S=3 / 2$ which the fit yields $J_{e} / k_{B}=18 \mathrm{~K}$. The solid red line is fit to Bloch law. To validate the calculated value of $J_{e}$ from Bloch law, it is also determined from molecular field theory (equation(3-37)) using molecular field theory, yielding $J_{e} / k_{B}=3 T_{\mathrm{C}} /[2 Z J(J+1)]$. With $Z=6$ as the number of exchange-coupled nearest neighbors each with spin $J=3 / 2$ and $T_{\mathrm{C}}=305 \mathrm{~K}$ for the $7.6 \mathrm{~nm}$ LSMO film gives $J_{e} / k_{B}=20 \mathrm{~K}$ close to $J_{e} / k_{B}=18 \mathrm{~K}$ determined from Bloch law. The green dashed line is fit to the molecular field theory. 


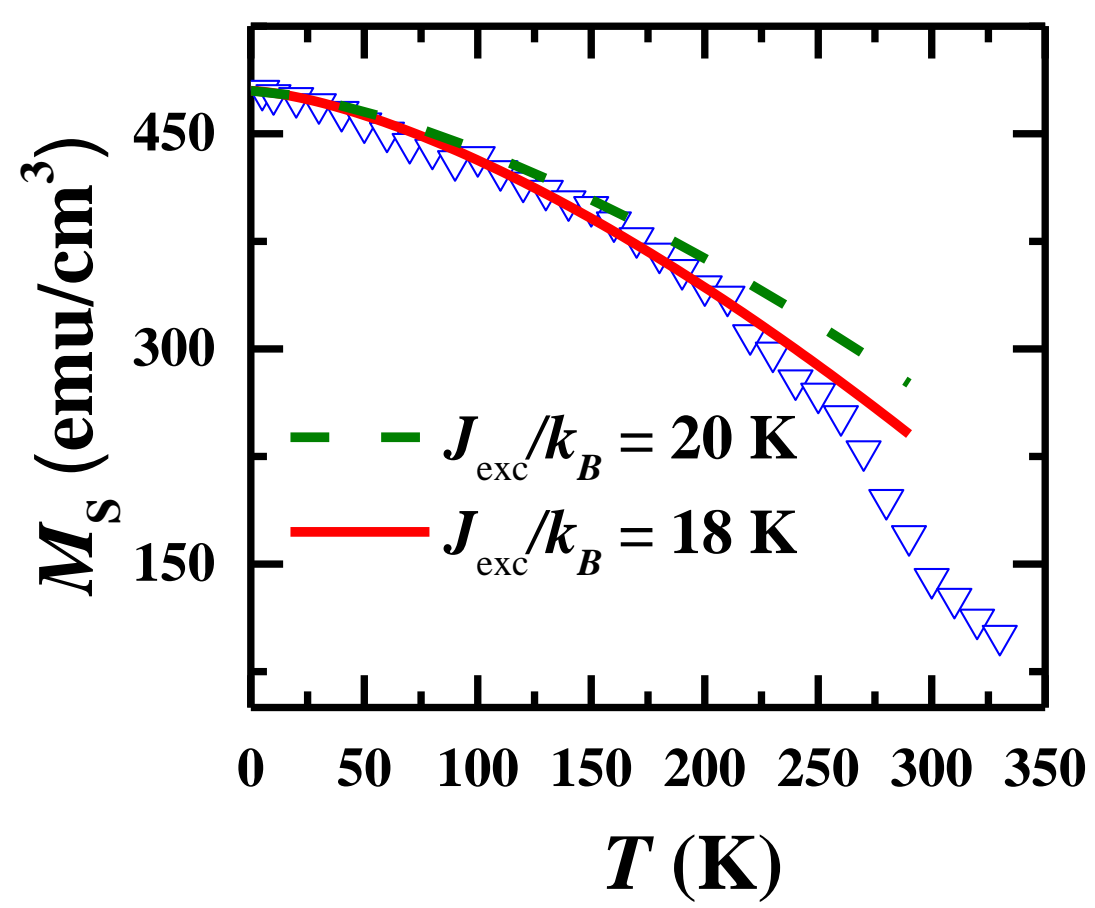

Figure 3.2. Temperature variation of $M_{\mathrm{S}}$ taken from corrected hysteresis loops [24].

The $M_{\mathrm{S}}$ vs. $T$ graph behaves like many other FM materials [66-68], however, the value of $M_{\mathrm{S}}$ in $7.6 \mathrm{~nm}$ LSMO/STO in $T=5 \mathrm{~K}$ is $476\left(\mathrm{emu} / \mathrm{cm}^{3}\right)$ which is lower than its bulk value $M_{\mathrm{S}}=583\left(\mathrm{emu} / \mathrm{cm}^{3}\right)$ because of the dead layer problem. The dead layer thickness in this sample can be calculated by the proposed dead layer model in equation(1-5). For $M_{\mathrm{S}}(D=7.6 \mathrm{~nm})=476\left(\mathrm{emu} / \mathrm{cm}^{3}\right)$ and $M_{\mathrm{S}}(\mathrm{b})=583\left(\mathrm{emu} / \mathrm{cm}^{3}\right)$ gives the dead layer thickness $d=1.4 \mathrm{~nm}$.

The common method to measure the temperature variation of saturation magnetization $(M-T)$ of a FM material is in the presence of a high magnetic field (Figure 1.7), however, a FM material has a spontaneous magnetization $\left(M_{\mathrm{SP}}\right)$ with all magnetic moments align in the same direction below $T \ll<\mathrm{T}_{\mathrm{C}}$ without exposing to an external magnetic field. Therefore, the purpose of using high magnetic fields is to align all the magnetic moments of a FM material that are not perfectly aligned in the same direction. To understand the effect of applied magnetic field on magnetization, I performed $M-T$ in low $(H=50$ Oe $)$ and high $(H=1$ $\mathrm{kOe}$ ) applied magnetic fields which is discussed in the next section.

\subsubsection{Temperature Variation of Magnetization and Blocking Temperature}

My observation on the temperature variation of magnetization ( $M$ vs. $T$ ) is shown in Figure 3.3. The measurements were done in $H=50,100,200,500 \mathrm{Oe}$, and $1 \mathrm{kOe}$. For the ZFC case, the sample was cooled to $5 \mathrm{~K}$ in $H=0$ Oe and measuring $H$ was then applied and $M$ vs. $T$ data taken up to $400 \mathrm{~K}$. For the FC case, the sample is cooled to $5 \mathrm{~K}$ in non-zero $H$ and $M$ vs. $T$ data taken similarly up to $400 \mathrm{~K}$ in the cooling $H$. 
Note the negative magnetization for the ZFC cases below $100 \mathrm{~K}$ for $H=50$ Oe and 100 Oe. For $H=200$ Oe and higher $H$, only positive values of $M(\mathrm{ZFC})$ are observed.

Due to the procedure utilized, the presence of negative magnetization in LSMO films was not observed in Figure 1.7 and this magnetic system was assumed to be a pure FM material and high magnetic fields were used to achieve the highest $M_{\mathrm{S}}$ value. Measurements in high applied magnetic fields up to $H=10 \mathrm{kOe}$ ( = $1 \mathrm{~T}$ ) do not exhibit negative magnetization and the applied field increases the $M_{\mathrm{S}}$ of the samples (Figure 1.7). The field magnitude ( $H=50 \mathrm{Oe})$ used in $M$ vs $T$ measurements of Figure 3.3 is much smaller $(0.5 \%)$ compared with the $H$ used in Figure 1.7 .

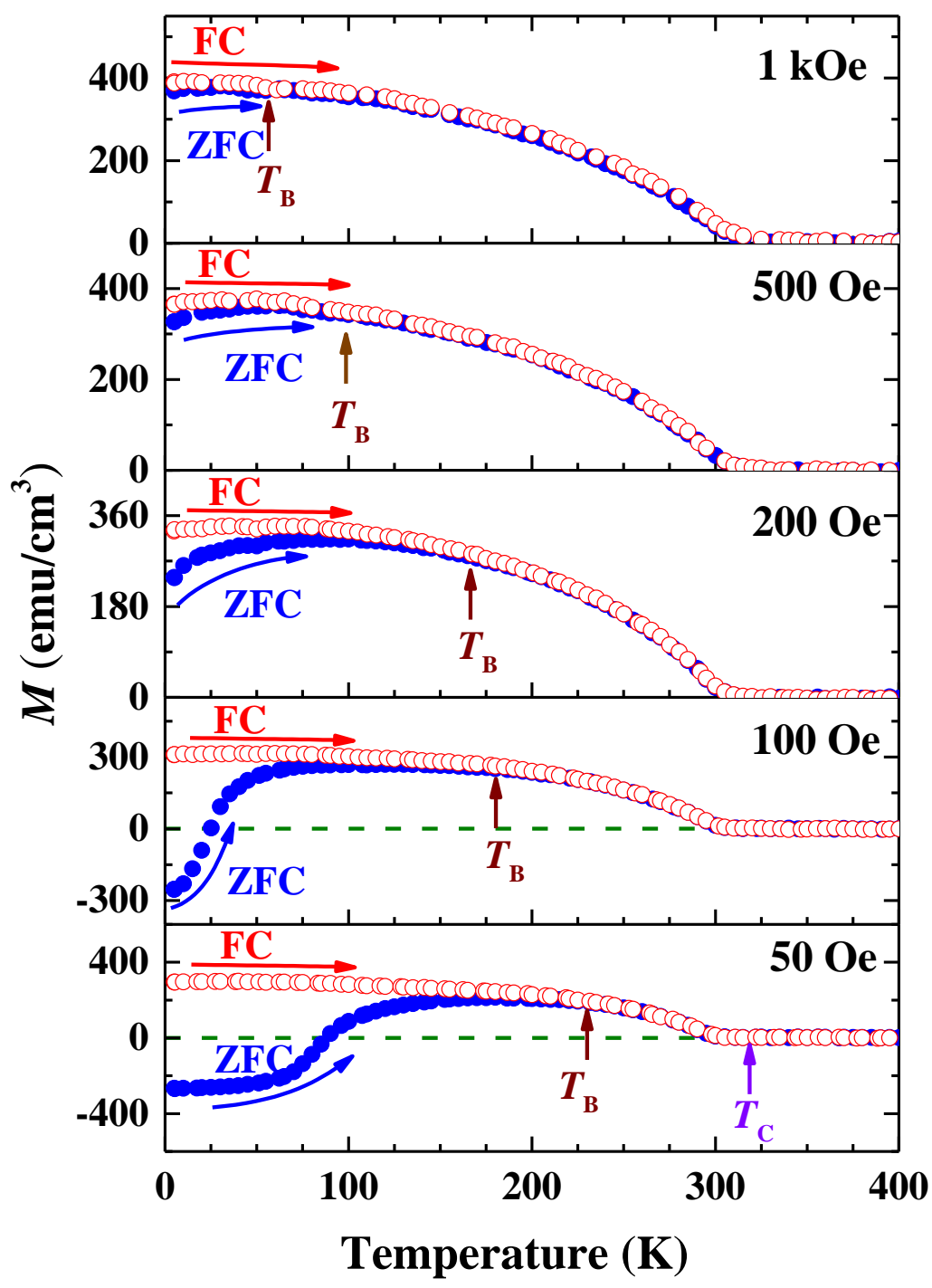

Figure 3.3. $M$ vs. $T$ data for the $7.6 \mathrm{~nm}$ LSMO/STO sample measured with $H=50,100,200,500$ Oe, and $1 \mathrm{kOe}$. Arrows mark the bifurcation temperature $T_{\mathrm{B}}$ separating the $M(\mathrm{FC})$ data (open red circles) from the $M$ (ZFC) data (closed blue circles). $T_{\mathrm{C}}$ is the Curie temperature [24]. 
In the ZFC cycle, the sample is cooled in no $H$ and it is measured in warming upcycle in the presence of $H$, and all magnetic moments are parallel with respect to the applied field direction and the material shows unblocked regions; whereas, in the FC cycle, the magnetic material is cooled and measured in $H$, and both blocked and unblocked regions are present. Blocking temperature $T_{\mathrm{B}}$ defined here by the bifurcation of the $M(\mathrm{FC})$ from the $M(\mathrm{ZFC})$ data represents the temperature above which all spins are unblocked and $T_{\mathrm{B}}$ decreases with increasing $H$. Below $T_{\mathrm{B}}, M$ decreases by decreasing the temperature and as expected, $T_{\mathrm{B}}$ is less than $T_{\mathrm{C}}$. The $T_{\mathrm{C}}$ is defined by the inflexion point in the $M$ vs. $T$ data. The decrement of $T_{\mathrm{B}}$ by increasing the $H$ can be explained by $T=T_{\mathrm{B}}\left(1-H / H_{\mathrm{C}}\right)^{2}(3-49)$ which by increasing the $H$ the $T$ decreases.

The presence of $T_{\mathrm{B}}$ is indicative of spin clusters. The size of spin clusters can be estimated by knowing the anisotropy constant of the film. The anisotropy and coercivity are related by $H_{\mathrm{C}} \sim K / M_{\mathrm{S}}$, where $H_{\mathrm{C}}$ and $M_{\mathrm{S}}$ are the coercive fields and saturation magnetization calculated from Figure 3.1, respectively. Using $M_{\mathrm{S}}$ $=476 \mathrm{emu} / \mathrm{cm} 3$ and $H_{\mathrm{C}} \approx 150 \mathrm{Oe}$, yields $\mathrm{K}=7.2 \times 10^{4} \mathrm{ergs} / \mathrm{cm}^{3}$. From equation-(3-44) the volume of the spin clusters is $V \approx 25 k_{\mathrm{B}} T_{\mathrm{B}} / K$. Using $T_{\mathrm{B}}=230 \mathrm{~K}$ calculated from $H=50$ Oe measurement in Figure 3.3 gives $V=1.1 \times 10^{-17} \mathrm{~cm}^{3}$. Assuming the shape of spin clusters is the cylindrical shape $V=D_{0}^{2} d$ as the $d(=1.4 \mathrm{~nm})$ is the thickness of the dead layer calculated from the equation-(1-5), gives $D_{0} \approx 90 \mathrm{~nm}$ as the diameter of the spin cluster [24]. This size is the approximate width of the magnetic domains in our films.

The values of $T_{\mathrm{B}}$ at different applied fields are calculated from Figure 3.3 and plotted as a function of $H$ in Figure 3.4. The dependence of $T_{\mathrm{B}}$ on $H$ shows that $T_{\mathrm{B}}$ decreases by increasing $H$. The field variation of $T_{\mathrm{B}}$ is seen to fit very well to equation-(3-49): $T_{\mathrm{B}}(H)=T_{\mathrm{B}}(0)\left[1-\left(H / H_{0}\right)\right]^{2}$. The value of $T_{\mathrm{B}}(0)=230 \mathrm{~K}$ which is very close to the value of $T_{\mathrm{B}}$ measured at $H=50$ Oe in Figure 3.3. 


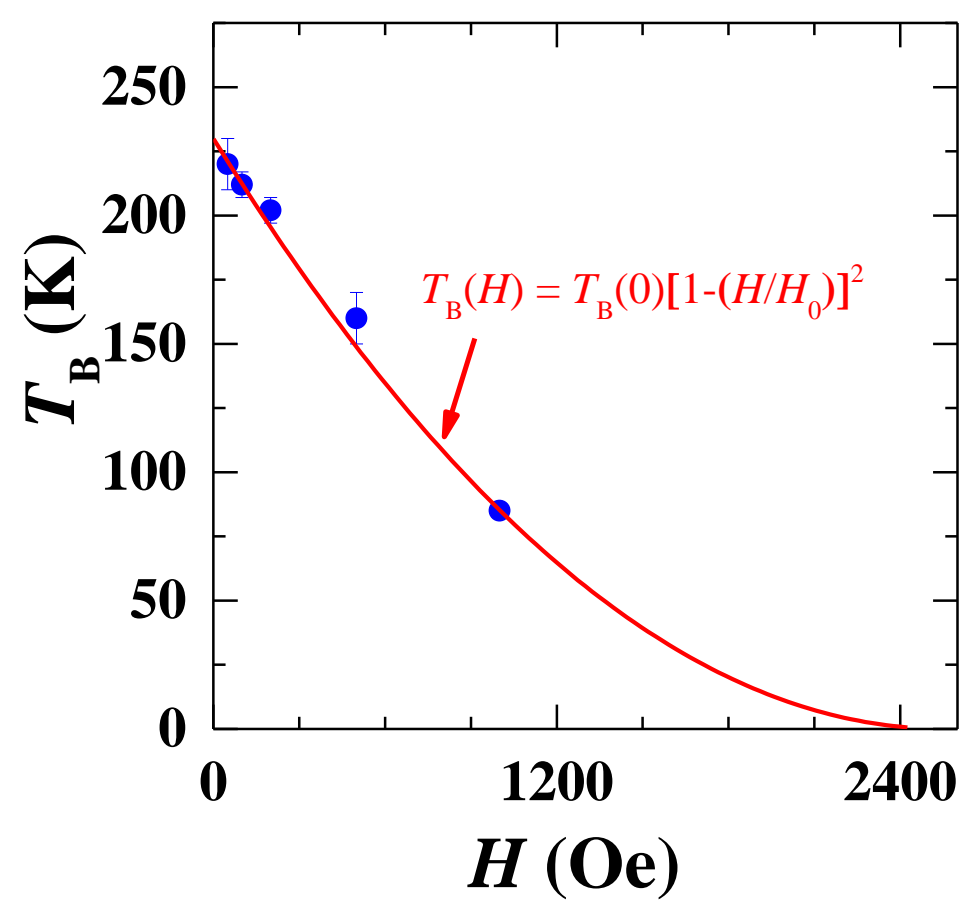

Figure 3.4. Variation of $T_{\mathrm{B}}$ with applied field $H$ [24].

\subsubsection{Temperature Dependence of Hysteresis Loops and Loop Parameters}

All the ZFC hysteresis loops (HLs) were done by cooling the samples from $360 \mathrm{~K}$ above the $T_{\mathrm{C}}$ of the sample to the measuring temperature in zero magnetic fields after demagnetizing the coils at $360 \mathrm{~K}$. All ZFC loops have three main cycles which include three field sweep directions which they start from $H=0$ Oe. Figure 3.5) shows a ZFC hysteresis loop at $T=190 \mathrm{~K}$ with filed sweep directions and loop parameters. The first cycle marked as \#1 starts from $H=0$ Oe to $H_{\max }=+4 \mathrm{kOe}$ and the second cycle (marked as \#2) starts from $H_{\max }=+4 \mathrm{kOe}$ to $H_{\min }=-4 \mathrm{kOe}$. In the final cycle, the field sweeps from $H=-4 \mathrm{kOe}$ to $H=$ $+4 \mathrm{kOe}$ to complete the field cycle. Figure 3.5 shows a ZFC hysteresis loop at $T=190 \mathrm{~K}$ with the field cycles and important magnetic characteristics which are defined as follows.

There are a few interesting features of note in our hysteresis loops. $M_{\mathrm{r}}(\mathrm{i})$ is the initial remanent magnetization which is the magnetization value of the sample at $H=0$ Oe in cycle \#1. It is normally zero, but our films start with a negative magnetization as soon as a small field is turned on. Thus, you could say that an initial coercivity $\left(H_{\mathrm{C}}(\mathrm{i})\right)$ is required for the field to switch the magnetization direction in cycle \#1. The $M_{\mathrm{r}}$ is the remanent magnetization which is defined as the value of magnetization at $H=0$ Oe after the sample was saturated in $H=4 \mathrm{kOe}$ in cycle $\# 2 . H_{\mathrm{C}}$ is the coercivity that is calculated by this relation $H_{\mathrm{C}}=$ $\left(H_{3}-H_{2}\right) / 2$ where $H_{2}$ and $H_{3}$ are the coercive fields calculated from cycles 2 and 3, respectively. 


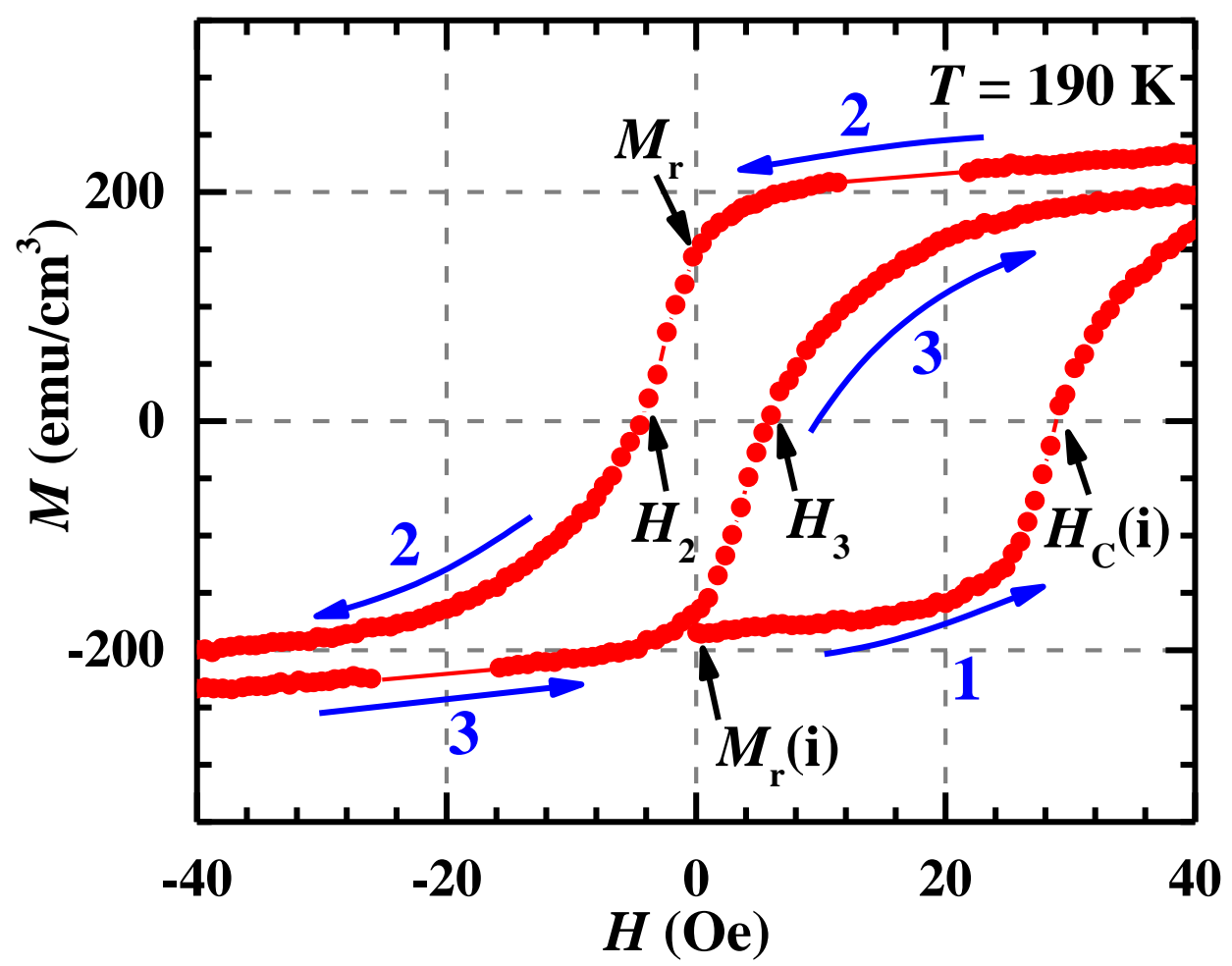

Figure 3.5. The low field zoom of the HL was measured at $190 \mathrm{~K}$ in the ZFC sample of $7.6 \mathrm{~nm}$ LSMO/STO. Arrows are marked as 1,2, and 3 to present the counter-clockwise cycle of the loop [69].

The cycle \#1 of the HL has $M_{\mathrm{r}}(\mathrm{i})<0$ and $H_{\mathrm{C}}(\mathrm{i})<0$ as measurements start from $H=0$ Oe, eventually yielding positive saturation magnetization at $H_{\max }$. In cycle \#2, the $M_{\mathrm{r}}>0$ where field sweeps from $H_{\max }$ to negative saturation magnetization at $H_{\min }$ with $H_{2}<0$. Likewise, cycle \#3 has $H_{3}>0$. This counterclockwise cycle of the loop for cycles 2 and 3 is a normal behavior of the hysteresis loop. The values of $M_{\mathrm{r}}$, $M_{\mathrm{r}}(\mathrm{i}), H_{\mathrm{C}}(\mathrm{i})$, and $H_{\mathrm{C}}$ are calculated for all temperatures.

The temperature dependence of these loop parameters, plotted in Figure 3.6, illustrates more interesting features. At $T=240 \mathrm{~K}$ the $M_{\mathrm{r}}$ and $H_{\mathrm{C}}=0$ which shows similar behavior to the SPM phase. As discussed in sections 1.2.3.2 and 3.3.1, the SPM material at $T_{\mathrm{B}}$ has $M_{\mathrm{r}}$ and $H_{\mathrm{C}}=0$ Oe thus $T=240 \mathrm{~K}$ is defined as $T_{\mathrm{B}}$ in these measurements. Whereas at $T=290 \mathrm{~K}, M_{\mathrm{r}}(\mathrm{i})=0, H_{\mathrm{C}}(\mathrm{i})=0$ and $H_{\mathrm{C}}=0$ which is the signature of a FM materials [4] at Curie temperature therefore $T=290 \mathrm{~K}$ is $T_{\mathrm{C}}$. The presence of $T_{\mathrm{B}}$ and $T_{\mathrm{C}}$ temperatures in this sample creates three distinct temperature regions; (i) $T<T_{\mathrm{B}}, T_{\mathrm{B}}<T<T_{\mathrm{C}}$ and (iii) $T>T_{\mathrm{C}}$. 

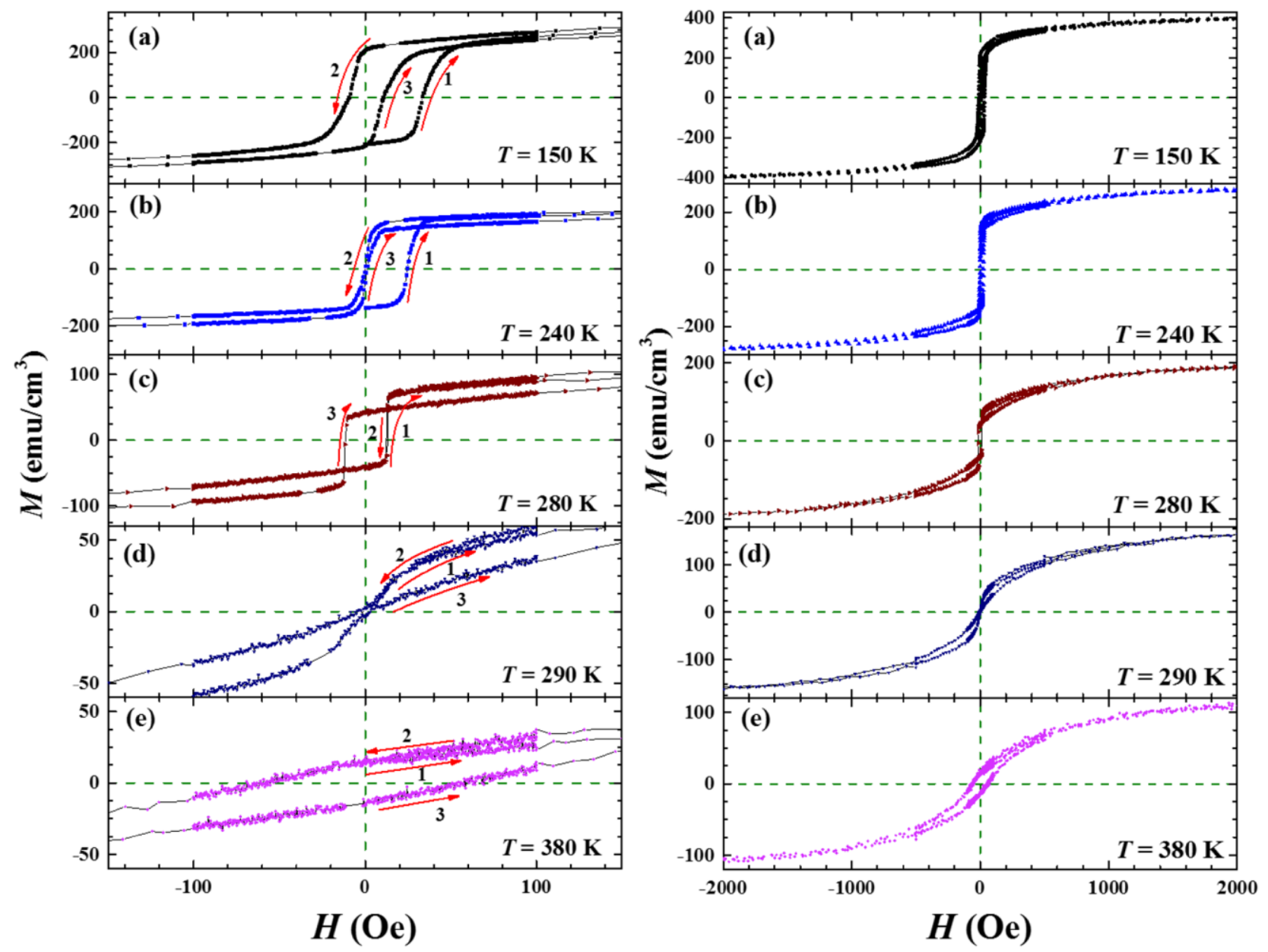

Figure 3.6. (Left figure) low field zoom in of the HLs for the ZFC sample of $7.6 \mathrm{~nm} \mathrm{LSMO/STO}$ at different temperatures (a) $T<T_{\mathrm{B}}$, (b) $T=T_{\mathrm{B}}$, (c) $T_{\mathrm{B}}<T<T_{\mathrm{C}}$, (d) $T=T_{\mathrm{C}}$, (e) $T>T_{\mathrm{C}}$. Arrows are marked as 1,2 , and 3 to present the direction of the magnetic field sweep. (Right figure) the same data are plotted up to $H= \pm 2000$ Oe to show loop closing which occurs at about $H=1000$ Oe [69].

There is a distinct change in the shape of the loops for the three regions noted above. In Figure 3.6 (left figure), I plotted the zoom in on the low-field range of the HLs for $T=150 \mathrm{~K}\left(T<T_{\mathrm{B}}\right), T=240 \mathrm{~K}\left(T=T_{\mathrm{B}}\right)$, $T=280 \mathrm{~K}\left(T_{\mathrm{B}}<T<T_{\mathrm{C}}\right), T=290 \mathrm{~K}\left(T=T_{\mathrm{C}}\right)$ and $T=380 \mathrm{~K}\left(T>T_{\mathrm{C}}\right)$. Also plotted in Figure 3.6 (right figure) are the zoomed-out loops for -2000 Oe $\leq H \leq 2000$ Oe at the same temperatures showing that the loops are closed at about $H=1000$ Oe. From the data plotted in Figure 3.6, it is evident that for $T=T_{\mathrm{B}}=240 \mathrm{~K}$ and $T=T_{\mathrm{C}}=290 \mathrm{~K}$, both $H \mathrm{C}$ and $M \mathrm{r}$ are effectively zero. In the region defined by $T_{\mathrm{B}}<T<T_{\mathrm{C}}$ such as the loop shown at $280 \mathrm{~K}$, the loops are inverted. The inverted hysteresis loops (IHLs) can be seen from the trends of segments \# 2 and \# 3 . At this temperature, segment 2 has $M_{\mathrm{r}}<0$ and the loop has a clockwise cycle, meaning that the magnetization switches to negative before the field is completely reduced to zero. 
This is in contrast with the HLs at $T=190 \mathrm{~K}$ shown in Figure 3.5 in which the second segment has $M_{\mathrm{r}}>0$ with a counter-clockwise cycle. This comparison confirms the HL inversion at $T=280 \mathrm{~K}$.

Furthermore, the temperature dependence of these loop parameters is plotted in Figure 3.7. In figure 3.7-b, $M_{\mathrm{r}}<0$ and $H_{\mathrm{C}}<0$ in the region of $T_{\mathrm{B}}<T<T_{\mathrm{C}}$ which confirms inversion of the HL, on the other hand, for $T>T_{\mathrm{C}}$ such as the loop shown at $380 \mathrm{~K}$ in Figure 3.6, we observe a normal loop with both $M_{\mathrm{r}}(\mathrm{i})$ and $M_{\mathrm{r}}$ being positive but small and nearly equal. From Figure 3.7, note that for $T<T_{\mathrm{C}}, M_{\mathrm{r}}(\mathrm{i})$ is negative and it becomes positive for $T>T_{\mathrm{C}}$. Thus the anomalous results reported here include the following: (i) the observation of NRM i.e. negative $M_{\mathrm{r}}(\mathrm{i})$ for $T<T_{\mathrm{C}}$ and becoming positive for $T>T_{\mathrm{C}}$ with temperature dependence below $T_{\mathrm{C}}$ qualitatively similar to that of saturation magnetization (Figure 3.7-e); (ii) the observation of IHLs for $T_{\mathrm{B}}<T<T_{\mathrm{C}}$ along with negative $H_{\mathrm{C}}$ and negative $M_{\mathrm{r}}$ (Figure 3.7-b); (iii) observation of positive $M_{\mathrm{S}}$ and $H_{\mathrm{C}}$ for $T>T_{\mathrm{C}}$ (Figure 3.7-b, c), a region normally defined by $M_{\mathrm{S}}=H_{\mathrm{C}}=0$ in a ferromagnet. 


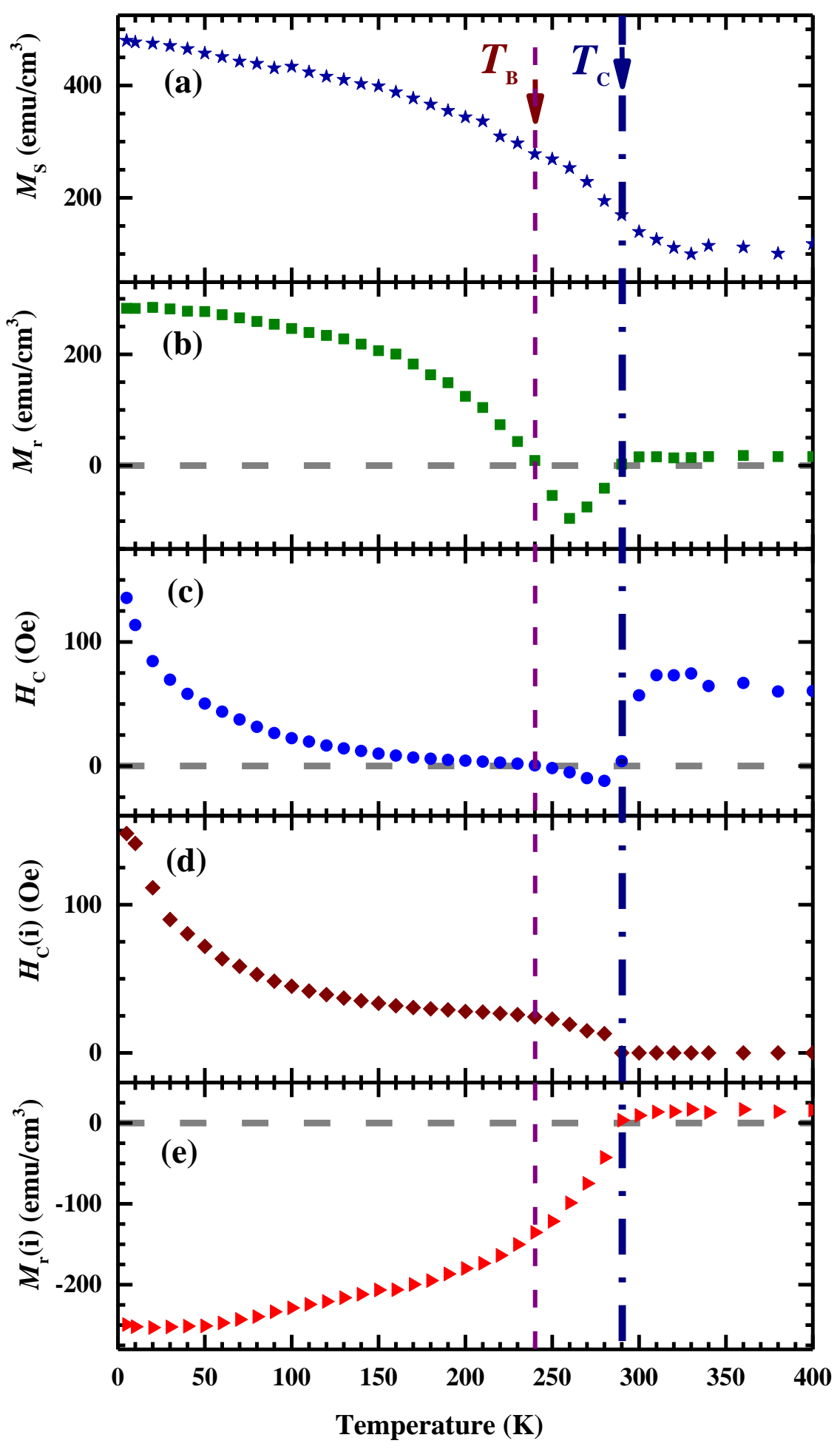

Figure 3.7. Temperature variations of $M_{\mathrm{S}}, M_{\mathrm{r}}, H_{\mathrm{C}}, H_{\mathrm{C}}(\mathrm{i})$, and $M_{\mathrm{r}}(\mathrm{i})$ defined in figure Figure 3.5 measured in the sample of $7.6 \mathrm{~nm}$ LSMO/STO. The positions of the blocking temperature $T_{\mathrm{B}}$ and the Curie temperature $T_{\mathrm{C}}$ are marked with vertical lines [69]. 
The presence of spin clusters in this sample is detected by temperature variation of magnetization (Figure 3.3) and the size of clusters is already calculated to be $D_{0} \sim 90 \mathrm{~nm}$. The hysteresis loops show that the existence of three distinct temperature regions suggests that this sample is magnetically inhomogeneous (MI) consisting of two magnetic phases: SPM and FM with the transition temperature at $T_{\mathrm{B}}=240 \mathrm{~K}$ and $T_{\mathrm{C}}=290 \mathrm{~K}$, respectively. Also, it is well known that IHLs and negative remanent magnetization (NRM) are not allowed in magnetically homogeneous systems since it violates the first law of thermodynamics [70, 71]. The presence of the SPM phase is likely caused by either clustering of oxygen vacancies and/or dislocations. This inhomogeneity along with FM ordering combined with short-range 2D ordering above $T_{\mathrm{C}}$ are the likely sources of the anomalous results noted above.

The existence of the FM and SPM phases in this sample creates a magneto-static interaction between the phases leading into NRM and IHL which can be explained by the total energy of this MI system can be written as [72]

$$
\begin{aligned}
E= & -M_{1} V_{1} H \cos \left(\theta_{1}-\theta_{H}\right)-M_{2} V_{2} H \cos \left(\theta_{2}-\theta_{H}\right) \\
& +K_{1} V_{1} \sin ^{2}\left(\theta_{1}\right)+K_{2} V_{2} \sin ^{2}\left(\theta_{2}\right)-J M_{1} M_{2} \cos \left(\theta_{1}-\theta_{2}\right),
\end{aligned}
$$

where $K, V$, and $M$ are the anisotropy constants, volume, and saturation magnetization of each magnetic phase. $H$ is the magnitude of the applied field, $\theta_{1}$ and $\theta_{2}$ are the angles of $M_{1}$ and $M_{2}$ with respect to the easy axis of magnetization of both phases. $\theta_{H}$ is the angle between $H$ and the easy axis of $M$. The last term is the magnetic interaction between the two phases. Assuming the $H$ is applied in the easy axis direction then $\theta_{H}=0$. The minimum energy of the system for $\theta_{1}$ and $\theta_{1}$ yields the four cases: (1) $\theta_{1}=\theta_{2}=0$, (2) $\theta_{1}=\pi, \theta_{2}=0$, (3) $\theta_{1}=0, \theta_{2}=\pi$, and (4) $\theta_{1}=\theta_{2}=\pi$.

The direction of $M$ in each magnetic phase depends on the magnitude of $H$ and the anisotropy of the sample. In high $H$ both $M_{1}$ and $M_{2}$ are aligned in the direction of $H$ that case- 1 is achieved. When the magnitude of $H$ decreases the total magnetization decreases and the spins of the SPM phase start to rearrange in the vicinity of the FM phase and due to the antiferromagnetic coupling between these phases and the most magnetic stable state is case-3. When the magnitude of $H$ decreases to zero meaning the $H$ direction reverses the SPM spins closer to the FM phase stay antiparallel but the magnetic moments of SPM which are far from the FM start to fluctuate and will be randomly oriented due to the magnetic nature of the SPM phase. The spins of the FM phase stay in the same direction $\left(\theta_{1}=0\right)$ due to large anisotropy. The amount of SPM phases is larger than the FM phase in $H=0$ thus the $M_{\mathrm{r}}<0$ and IHL occurs in $T_{\mathrm{B}}<T<T_{\mathrm{C}}$.

NRM and IHLs have been reported in the (Ni-Fe)-SiO2 films [73] and in randomly distributed Co-NP systems containing NPs of $4 \mathrm{~nm}$ and $25 \mathrm{~nm}$ sizes [72]. In both these systems, a FM phase along with a SPM phase was shown to co-exist and the observed NRM and IHL were interpreted to be due to the negative 
magneto-static interaction (dipolar or exchange). between the FM and the SPM phases. For the (Ni-Fe)$\mathrm{SiO} 2$ films, two phases were detected for about $50 \%$ volume fractions of the magnetic NPs whereas for the Co-NP system, the SPM phase was attributed to the smaller $4 \mathrm{~nm}$ NPs and the FM phase to the larger 25 nm NPs [72].

For the $7.6 \mathrm{~nm}$ LSMO/ STO film, our magnetic measurements have established the co-existence of the SPM-FM phases in the temperature region of $T_{\mathrm{B}}<T<T_{\mathrm{C}}$, the same region where we observe IHL. For $T<$ $T_{\mathrm{B}}$, the spins in the SPM state are blocked/frozen and although we still observe negative initial $M_{\mathrm{r}}(\mathrm{i})$ in this region, the loops are not inverted, and hence positive $M_{\mathrm{r}}$ and $H_{\mathrm{C}}$ are observed. This inhomogeneity along with FM ordering combined with short-range $2 \mathrm{D}-\mathrm{FM}$ short-range ordering above $T_{\mathrm{C}}$ are the likely sources of the anomalous results noted above. Therefore, the observation of positive $M_{\mathrm{S}}, H_{\mathrm{C}}$, and $M_{\mathrm{r}}$, though small, well above $T_{\mathrm{C}}$ in the $7.6 \mathrm{~nm}$ LSMO/STO film is quantitatively attributed to the persistence of this $2 \mathrm{D}$ magnetic ordering. It has also been observed that substrates prepared in the same growth environment as the films can exhibit weak high-temperature magnetization, which may also induce magnetic behavior at the interface [74]. In the next section, I discuss the time viscosity measurements on this MI sample.

\subsection{Magnetic Relaxation Measurements: Magnetic Viscosity Investigation}

Magnetic viscosity is originated from thermal fluctuations of magnetic moments over energy barriers which can show the magnetization process of a magnetic sample [61]. The viscosity measurements follow the logarithmic relaxation behavior because of a distribution of energy barriers separating local minima in the free energy which can reflect different equilibrium states. As is shown in section 3.4 .3 the $7.6 \mathrm{~nm}$ LSMO/STO film is magnetically inhomogeneous. The co-existence of SPM and FM phases can produce a distribution of energy barriers in this sample which can be studied by viscosity measurements to study the relaxation process specifically in the temperature region of $T_{\mathrm{B}}<T<T_{\mathrm{C}}$.

The protocol used for magnetic viscosity measurements was as follows: after demagnetizing the magnet coil at $320 \mathrm{~K}$ using the oscillating mode to reduce the residual field $<2$ Oe, the sample was cooled to the measuring temperature (e.g., $5 \mathrm{~K}$ ) in dc magnetic field $H=50$ Oe. After temperature became stable, $H$ was switched to 0 Oe and time-dependent magnetization $M(t)$ was measured for time $t$ up to $2 \mathrm{~h}$. The sample was then warmed up to $320 \mathrm{~K}$, the coils were demagnetized again followed by cooling the sample to the next measuring temperature in $H=50$ Oe and repeating the $M(t)$ vs $t$ measurements after switching $H$ to zero. From the plot of $M(\mathrm{t})$ vs $\ln (t)$, the magnetic viscosity $S$ was determined using the Eq. (3-56): $M(t)=M(0)-S \ln (t)[75]$. 
Figure 3.8 shows the plot of $M(\mathrm{t})$ vs $\ln (t)$ for $\mathrm{t}$ up to 2 hours for selected temperatures between $5 \mathrm{~K}$ and $305 \mathrm{~K}$. The quantities of interest are $M(0)$, the slope of the plot, which according to the equation-(3-56) is the magnetic viscosity $S$ and $M$ (2 hours), the magnitude of the magnetization at the end of 2 hours.

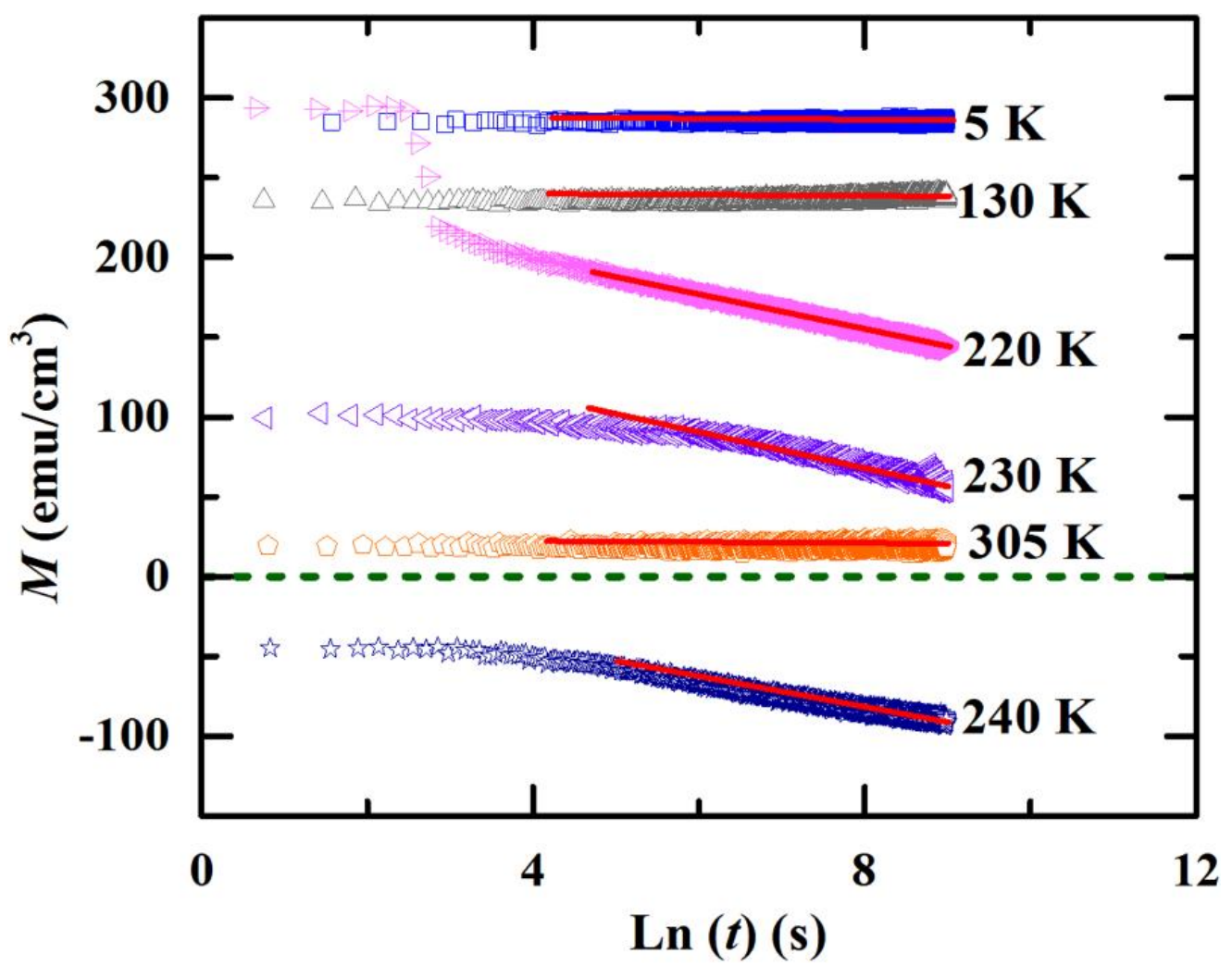

Figure 3.8. Variation of the magnetization $M(t)$ with time (the ln scale) at select temperatures after cooling the sample in $H=50$ Oe to the measuring temperature and then switching $H$ to zero. The solid lines are linear fits at higher times to determine viscosity $\mathrm{S}$ using the equation: $M(t)=M(0)-S \ln (t)$ [76].

Plots of $S$ vs $T$ and $M(2 \mathrm{~h})$ vs $T$ are shown in Figure 3.8 (a) and (b), respectively. The noteworthy new result is that $S$ peaks at $230 \mathrm{~K}$ above which $M$ (2 hours) switches from positive to negative values. With further increase in temperature, the magnitude of $M$ ( 2 hours) decreases, eventually becoming near zero above $T_{\mathrm{C}}$. Although a peak in $S$ vs $T$ has been reported in magnetic nanoparticles related to the spin-glass ordering of the surface $\mathrm{Fe}^{3+}$ spins [77] the accompanying switching of the sign of $M$ (2 hours) has not been reported in any other system before.

The unique result of Figure 3.9, viz., the peak in $S$ accompanied by sign switching of $M$ ( 2 hours), can be understood in terms of the similar temperature dependence of the hysteresis loop parameters. From $M$ vs $T$ and ZFC hysteresis loops (= Figure 3.8 and Figure 3.9) the magnetic phase competition between SPM and FM results in IHLs, $M_{\mathrm{r}}(\mathrm{i})<0, M_{\mathrm{r}}<0$, and $H_{\mathrm{C}}<0$. According to the equation-(3-57), the most stable state of this MI sample in no field requires the SPM phase to be antiparallel with respect to the FM phase 
$\left(\theta_{1}=0, \theta_{2}=\pi\right)$. The viscosity measurements were recorded in no field accordingly the system goes into this stable magnetic state results in negative magnetic relaxation.

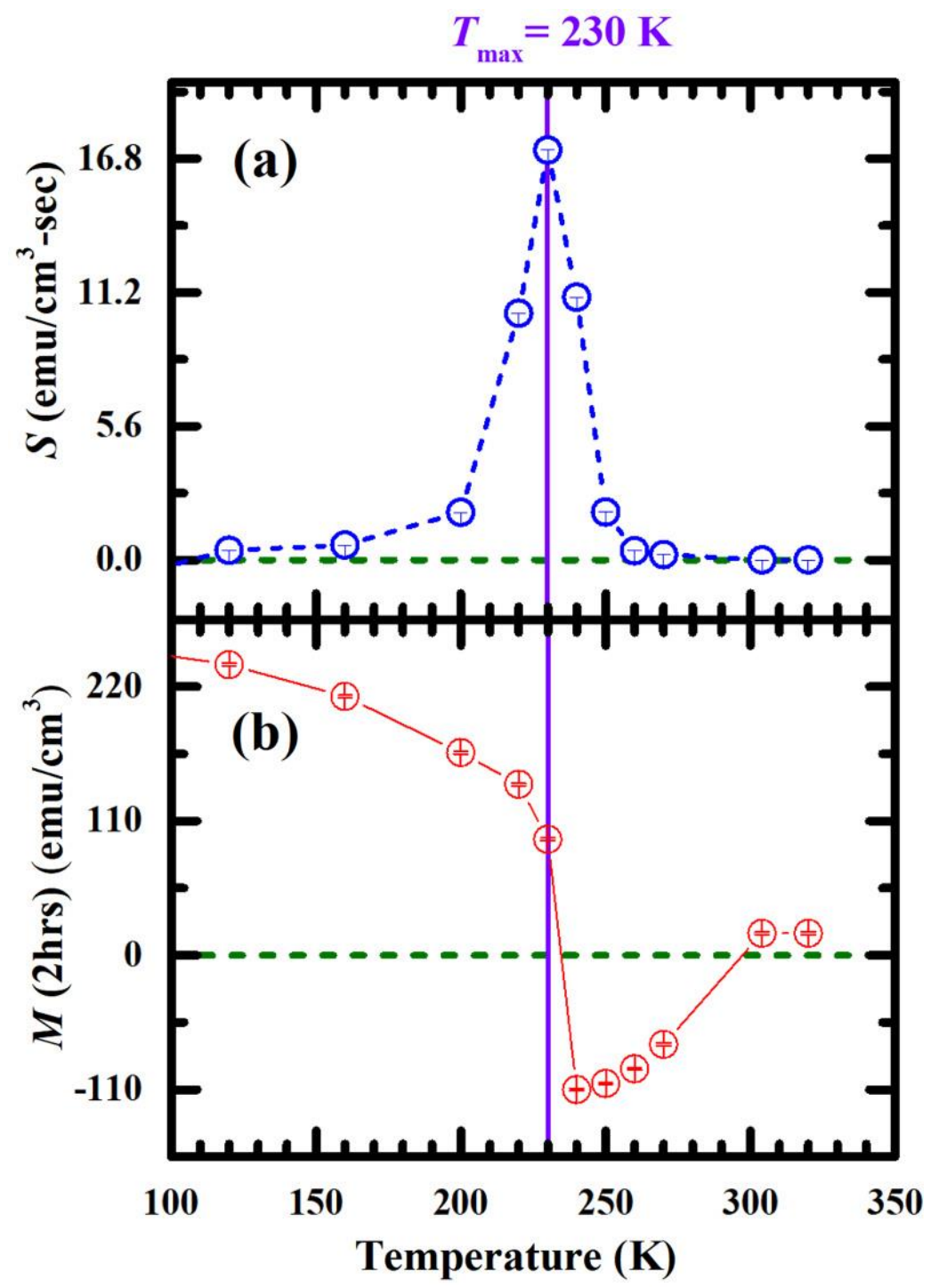

Figure 3.9. Temperature variations of the magnetic viscosity $S$ and (b) $M$ ( 2 hours) determined from the analysis of the data in Figure 3.8, $M$ (2 hours) being the measured $M$ at the end of 2 hours of time scan. The lines connecting the data points are visual guides [76].

\subsection{Summary}

In this chapter, I covered the important theoretical backgrounds such as spin waves, molecular field theory, characteristics of a SPM phase, Cole-Cole theory, Vogel-Fulcher law, and total energy of an MI 
magnetic system along with experimental results of a MI sample including $M-T$, hysteresis loops, and magnetic viscosity measurements. The theoretical backgrounds will be used in subsequent chapters especially in chapter 5 where the ac magnetic susceptibility results will be covered. From experimental results, I showed the important emergence of negative magnetization and negative magnetic relaxation which is caused by magnetic phase competition between the SPM and FM phases. In the subsequent chapter, I will discuss the thermodynamic properties of this sample in terms of magnetocaloric measurements which confirm the presence of the two magnetic phases: SPM and FM. 


\section{Chapter 4 \\ Magnetocaloric Studies}

\subsection{Introduction}

The discovery of a magnetic phenomenon that allows easy switching of a nearly saturated magnetic with a small magnetic field has many potential exciting applications. One potential example is magnetic refrigeration. In this chapter, the magnetocaloric results of a $7.6 \mathrm{~nm}$ LSMO/STO sample are discussed in detail. I will discuss the effects of magnetic inhomogeneity (MI) in a change of magnetic entropy and the relative cooling power of this magnetic system. The volume ratio of each magnetic phase is calculated in this chapter which is in good agreement with those calculated in chapter 3.

The results of this chapter have been published by the author recently (N. Mottaghi, et al, J. Alloys Compd. 154200 2020) and presented at American Vacuum Society (AVS 66th) and received the 2019 Leo Falicov Student Award for the best presentation of graduate research in this conference.

\subsection{Magnetic Field and Temperature Dependence of The Change of Magnetic Entropy}

Since we have at least two competing magnetic phases, it would be useful to learn more about how they interact over our temperature range. To determine the nature of the magnetic phase transition and the magnetocaloric (MC) properties of the sample, isothermal measurements of $M$ versus $H$ data presented in figure 4-1)-(a) from $210 \mathrm{~K}$ to $320 \mathrm{~K}$ in steps of $10 \mathrm{~K}$ were used. ZFC $M$ vs. $H$ measurements at different temperatures was obtained in the range of $0 \leq H \leq 4$ kOe using a Quantum Design physical property measurement system (PPMS-9T). The demagnetization process was performed by heating the sample above the magnetic ordering temperature. For each measurement, the magnet coil was first demagnetized in the oscillating mode so that the residual $H$ is reduced to $<2$ Oe. In the ZFC cycle, after demagnetizing the sample, it was cooled down to $5 \mathrm{~K}$ in $H=0$ Oe.

To determine the $T_{\mathrm{C}}$ and the nature of the magnetic phase transition, Arrot plots and Banerjee criterion were employed, respectively. Arrot plots are the curves of $M^{2}$ vs $H / M$ which are shown in figure 4-1)4.1b. The Curie temperature can be determined by fitting a linear line to $M^{2}$ vs $H / M$ graphs; if the intercept of a fitted line has zero value, then that value of $T$ is $T_{\mathrm{C}}[4,12]$. The $T_{\mathrm{C}}$ of this sample is $\sim 290 \mathrm{~K}$ shown by the solid red line in figure 4-1)Error! Reference source not found.-b. In this LSMO thin film, the slope of the graphs is positive thus the nature of the FM-PM phase transition is a second-order magnetic phase transition (SOMT) [78]. Additionally, it confirms the continuous FM-PM phase transition at $T_{\mathrm{C}}$ [79]. The obtained value of $T_{\mathrm{C}}$ with Arrot plots is in good agreement with the ZFC hysteresis loops (HLs) measurements to identify the $T_{\mathrm{C}}$ in Figure 3.6-d. 

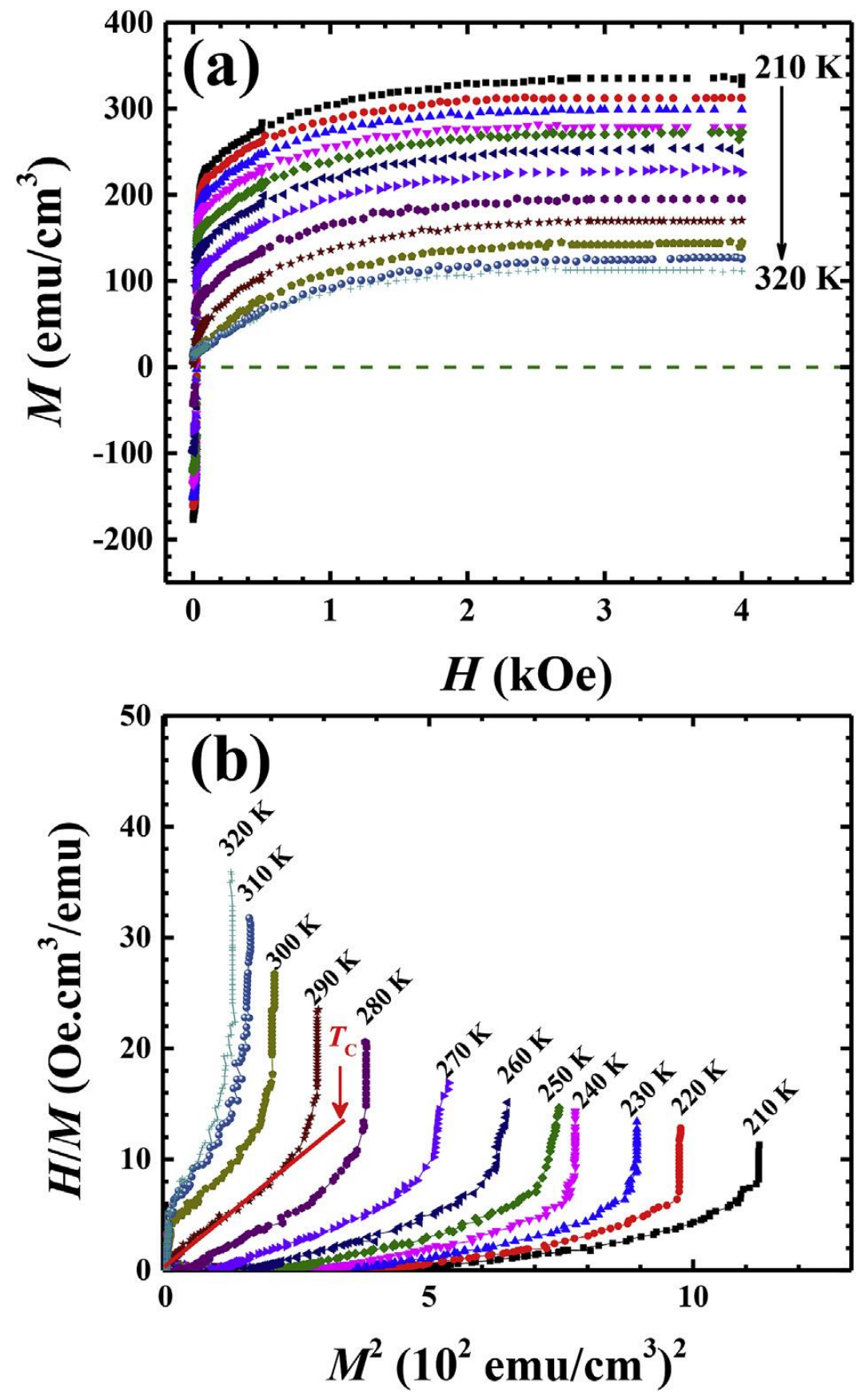

Figure 4.1. (a) Isothermal $M$ versus $H$ curves at selected temperatures from $210 \mathrm{~K}$ to $320 \mathrm{~K}$ in $10 \mathrm{~K}$ steps. (b) Arrott plot of the data at different temperatures yielding $T_{\mathrm{C}} \sim 290 \mathrm{~K}$ [80].

Based on the famous Maxwell relations, the isothermal magnetic entropy change, $\Delta S_{\mathrm{M}}(T, H)$, as a function of $H$, is given by 


$$
\Delta S_{M}(T, H)=S_{M}(T, H)-S_{M}(T, 0)=\int_{0}^{H}\left(\frac{\partial M\left(T, H^{\prime}\right)}{\partial T}\right)_{H^{\prime}} d H^{\prime}
$$

since $M$ is measured at discrete $H$ and temperature intervals, and $\Delta S_{M}(T, H)$ can be approximately calculated by the following equation

$$
\Delta S_{M}(T, H)=\sum_{i} \frac{M_{i+1}\left(T_{i+1}, H\right)-M_{i}\left(T_{i}, H\right)}{T_{i+1}-T_{i}} \Delta H
$$

where the magnetic entropy change at temperature $T$ is the difference of two isothermal magnetization curves at $T$ and $T+\Delta T$ divided by the $\Delta T$ (the temperature difference between two isothermal magnetizations) in figure Figure 4.1Error! Reference source not found.-a.

Normally in a homogeneous magnetic system, there is only one peak in the temperature variation of the magnetic entropy change graphs ( $-\Delta S_{\mathrm{M}}$ vs. $\left.T\right)$, which is around the transition temperature of the magnetic phases. For example, in magnetically homogeneous LSMO NPs [81] and LSMO films [82] the peak occurs around $T_{\mathrm{C}}$ where the FM-PM magnetic phase transition takes place. On the other hand, there might not be a single and sharp peak at the temperature variation of $\Delta S_{\mathrm{M}}$. This is true when a magnetic material is magnetically inhomogeneous or the surface-to-volume ratio of a sample is significantly large [83]. The MI causes a broadening and shifting of a peak in $-\Delta S_{\mathrm{M}}$ vs. $T$ graph [84]. Magnetocaloric measurements are useful methods to study the thermodynamic properties of the MI samples and compounds and identify the transition temperatures [85].

The temperature change of the magnetic entropy is shown in figure Figure 4.2. Two very broad peaks with considerable overlaps are present. The first peak is centered near $220 \mathrm{~K}$ which is very close to the temperature where the maximum viscosity occurs at $T_{\mathrm{B}}=330 \mathrm{~K}$ (Figure 3.9-a). The $T_{\mathrm{B}}$ was defined as the blocking temperature of the sample that the SPM-FM phase transition occurs. In sections 3.4.2 and 3.4.3, $T_{\mathrm{B}}$ was measured from hysteresis loops (HLs) and temperature variation of magnetization $(M-T)$. The second peak near $270 \mathrm{~K}$ is in the vicinity of the $T_{\mathrm{C}}$ of the sample. The $T_{\mathrm{C}}$ was measured by hysteresis loop measurements in section 3.4.3 (Figure 3.6-d). The observation of these two peaks in $-\Delta S_{\mathrm{M}}$ vs. $T$ graph are the new observation in the LSMO system which confirms the results of magnetic inhomogeneity in $M-T$, HLs, and magnetic viscosity measurements. The values of $\Delta S_{\mathrm{M}}$ in figure Figure 4.2 are comparable with those reported in other magnetic systems $[85,86]$. 

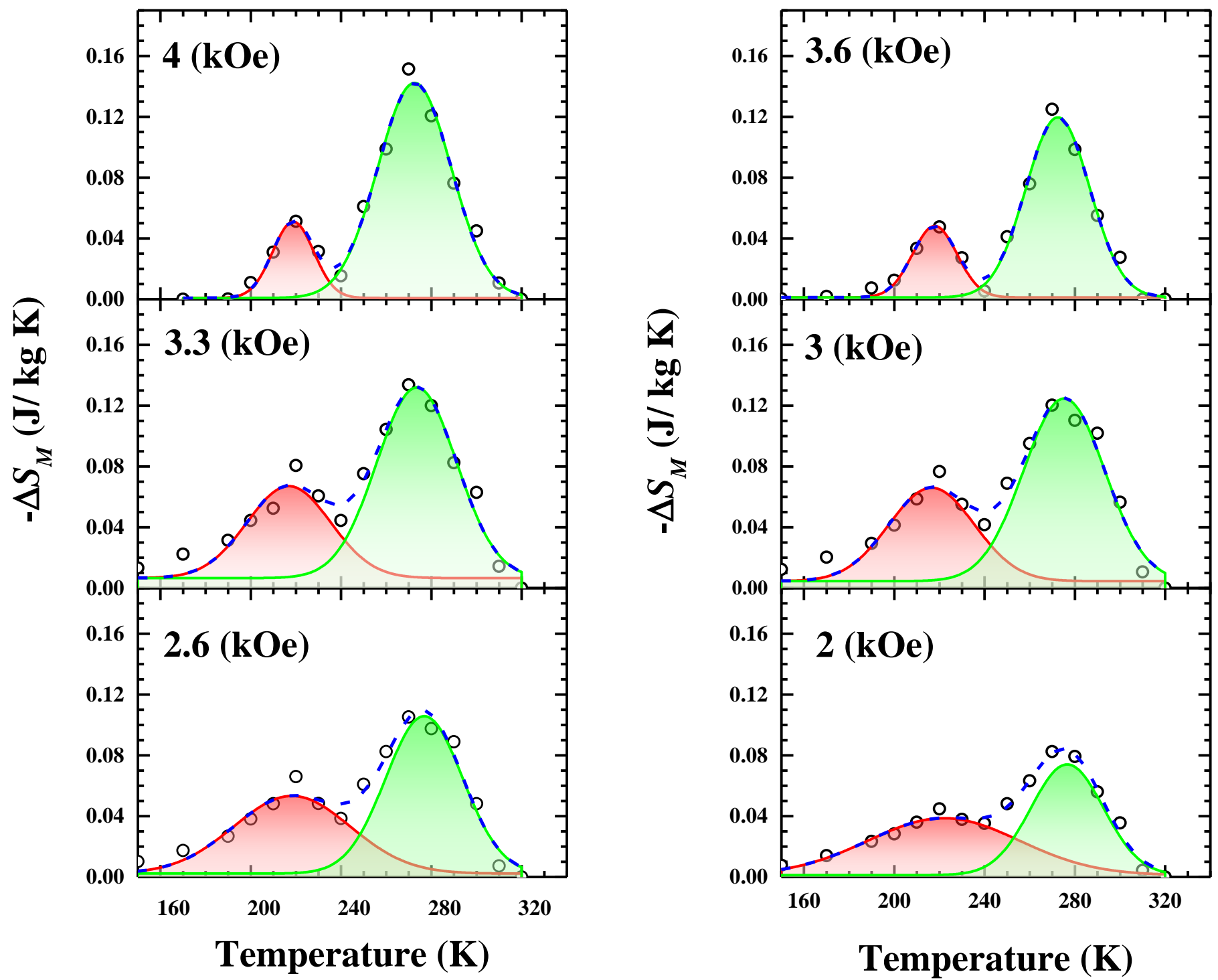

Figure 4.2. Magnetic entropy of the sample (open circles) versus temperature at different applied magnetic fields. The solid lines (red and green) are fit the two Gaussian functions and the blue dashed line is the sum of Gaussian functions [80].

\subsection{Magnetic Phase Volume Fraction Calculation From $\Delta S_{M}(T, H)$ vs. $T$ Graphs at Different $H$}

To gain further information from the two peaks observed in Figure 4.2, we fitted the two peaks to two Gaussian functions for all $H$ values shown in this figure. The $\Delta S_{M}(T, H)$ curves at different applied magnetic fields fitted two Gaussian functions. The $\Delta S_{M}(T, H=4(\mathrm{kOe}))$ for this sample can be obtained from the SPM and FM phases by 


$$
\left|\Delta S_{M}(T, H=4(\mathrm{kOe}))\right|=\left|\alpha \Delta S_{M, \text { SPM-FM }}(T, H=4(\mathrm{kOe}))+\beta \Delta S_{M, \text { FM-PM }}(T, H=4(\mathrm{kOe}))\right|
$$

where relation where $\alpha$ and $\beta(0 \leq \alpha, \beta \leq 1 ; \alpha+\beta=1)$ present the relative volume fractions of the SPM and FM phases. Magnitudes of $\alpha$ and $\beta$ are calculated from the area ratios of the two fitted Gaussian functions to the $\Delta S_{M}(T, H=4(\mathrm{kOe}))$ vs $T$ curve. Here $\Delta S_{M \text {, SPM-FM }}(T, H=4(\mathrm{kOe}))$ and $\Delta S_{M \text {, FM-PM }}(T, H=$ $4(\mathrm{kOe}))$ are change of magnetic entropy of SPM-FM and FM-PM magnetic phases transitions, respectively at $H=4(\mathrm{kOe})$. From the Gaussian function fit to the $-\Delta S_{M}(T, H=4(\mathrm{kOe}))$ vs. $T$, we obtain $\alpha=16.5 \%$ for the SPM phase and $\beta=83.5 \%$ for FM phase.

In section 3.4.1, the thickness of the magnetic dead layer containing the SPM spin clusters in this sample is calculated from the ZFC HL at $T=5 \mathrm{~K}$ (Figure 3.1) $M-T$ plots at $H=50$ Oe (Figure 3.3) which is around $1.4 \mathrm{~nm}$ and the remaining $6.2 \mathrm{~nm}$ as the thickness of the FM phase. Assuming these two phases are uniformly distributed through the surface of the sample meaning both have the same surface area then the volume of each magnetic phase is $V_{\mathrm{i}}=A d_{\mathrm{i}}$ where $A$ and $d_{\mathrm{i}}$, are the surface area of the sample and the thickness of each phase, respectively. The relative volume ratios with respect to the total volume of the sample are equal to the thickness ratios. The thickness of the sample is $D(=7.6 \mathrm{~nm})$ and the volume ratios of SPM and FM phases are the thickness ratios $d_{\mathrm{SPM}} / D$ and $d_{\mathrm{FM}} / D$, respectively. Therefore, the relative volume fractions of SPM and FM phases are $18 \%$ and $82 \%$ of the total volume of the sample, respectively which are in good agreement with the volume fractions calculated from the $-\Delta S_{\mathrm{M}}$ vs. $T$ graphs.

\subsection{Magnetic Field Dependence of the Change of Magnetic Entropy}

Magnetic field variation of $-\Delta S_{\mathrm{M}}$ is studied to quantify the magnetic state of the sample. To understand this behavior, the data in Figure 4.3-(a)Error! Reference source not found. is fit to an exponent power law: $-\Delta S_{M}=a H^{n}$ where ' $a$ ' is a constant and the exponent ' $n$ ' depends on the magnetic state of the sample $[88,89]$. Determination of the ' $n$ ' exponent allows us to find suitable theoretical models to explain the MCE in the system. It is predicted that the value of ' $n$ ' $=2$ in the Curie law above $T_{\mathrm{C}}$ and using the molecular field (MF) theory this value is predicted to be $2 / 3$ [90]. The Curie law approach employs Curie-Weiss behavior well above $T_{\mathrm{C}}$ to obtain the theoretical relationship between $-\Delta S_{M}$ and $H$; on the other hand, the MF theory uses reversion of power series to obtain solutions of magnetization near $T_{\mathrm{C}}$ [90].

It has been reported that the value of ' $n$ ' in polycrystalline and nanocrystalline manganites at the Curie temperature is $<1$ and in the ranges of $T<T_{\mathrm{C}}$ and $T>T_{\mathrm{C}}$ ' $n$ ' reaches 1 and 2 respectively [89]. Our fit of the data to this $-\Delta S_{M}=a H^{n}$ equation for several $T \leq T_{\mathrm{C}}$ in figure Figure 4.3-b shows $n \sim 1$ for $T<T_{\mathrm{C}}$ with the magnitude of $n$ increasing for $T>T_{\mathrm{C}}$. This deviation of $\mathrm{n}$ from $n=2 / 3$ is likely due to the presence of SPM spin clusters in the dead layer for $T<T_{\mathrm{C}}$. At $T_{\mathrm{B}}$, the value of $n$ is around 0.83 which is the signature of SPM 
phase in MC studies [89]. The larger magnitudes of $n$ for $T>T_{\mathrm{C}}$ are due to the Curie-Weiss variation of the magnetization in this region.
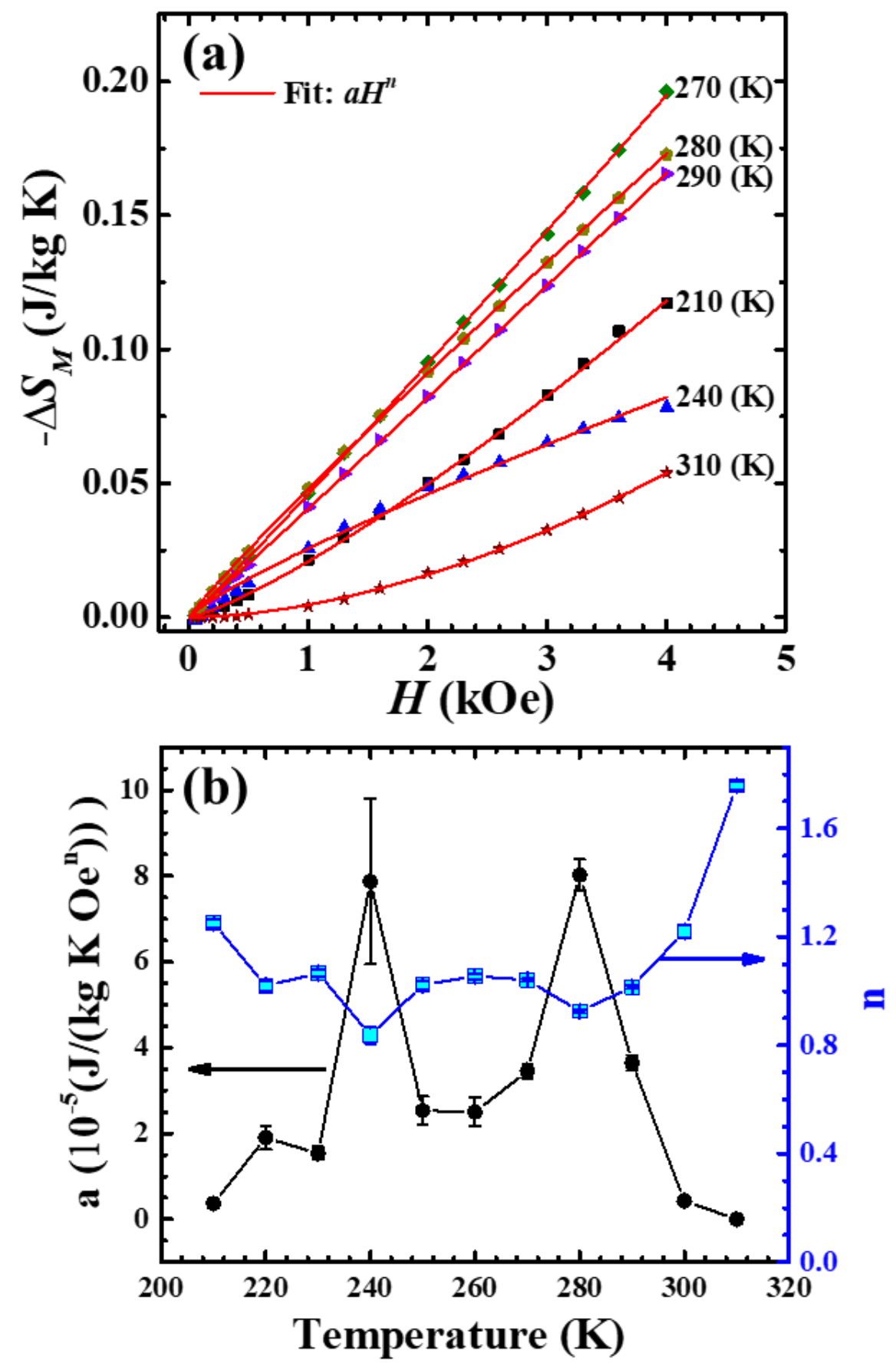

Figure 4.3. (a) Magnetic field dependence of the change in magnetic entropy at different temperatures. (b) Temperature dependence of ' $a$ ' and the exponent ' $n$ ' versus temperature [80]. 


\subsection{Specific Heat Calculation}

It is known that $\Delta S_{\mathrm{M}}(T, H)$ can also be obtained from the magnetic field dependence of the specific heat through the subsequent integration

$$
\Delta S_{M}(T, H)=\int_{0}^{T} \frac{C_{p}(T, H)-C_{p}(T, 0)}{T} d T,
$$

where $C_{p}(T, H)$ and $C_{p}(T, 0)$ are the measured heat capacity values in an applied field $H$ and zero applied field, respectively. From equation-(4-4) one can calculate the change of induced specific heat by an applied $H$ as [13].

$$
\Delta C_{p}(T, H)=C_{p}(T, H)-C_{p}(T, 0)=T \frac{\partial\left(\Delta S_{M}(T, H)\right)}{\partial T} .
$$

Using equation-(4-5), the temperature variation of $\Delta C_{p}$ of the sample is calculated at different applied $H$. Figure 4.4 shows broad anomalies centered around $T_{\mathrm{B}}$ and $T_{\mathrm{C}}$. With the increase in temperature, $\Delta C_{p}$ changes from negative to positive values showing a peak near $T_{\mathrm{B}} \sim 220 \mathrm{~K}$, then decrease again with a minimum near $250 \mathrm{~K}$, then increases again with a broad peak centered near $T_{\mathrm{C}}$. These variations are likely related to the negative remanence reported previously by us in this sample in the temperature range of $T_{\mathrm{B}}$ to $T_{\mathrm{C}}$ discussed in sections 3.4.2 and 3.4.3. 


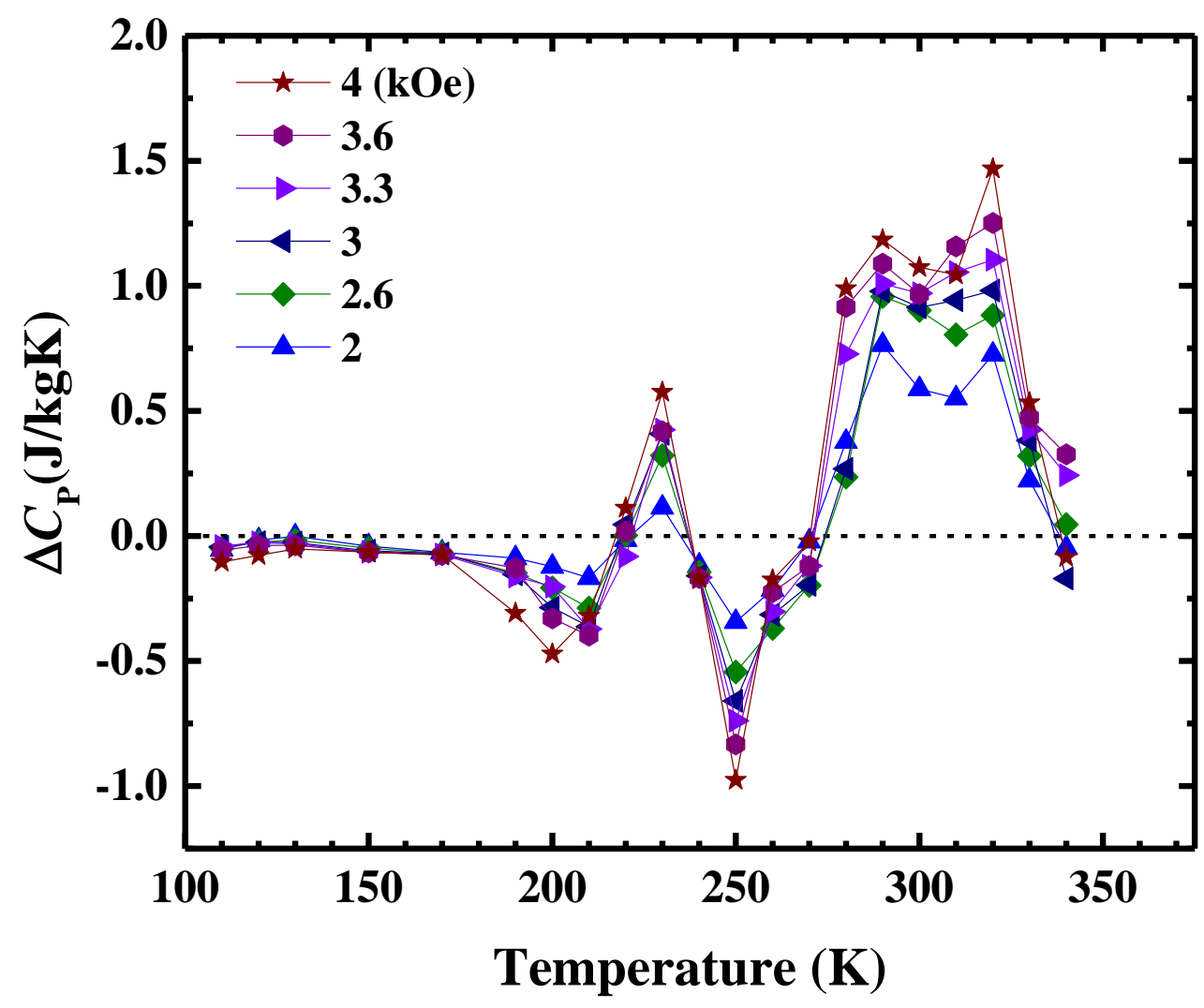

Figure 4.4. Calculated temperature dependence of specific heat of the sample in different magnetic fields from $2 \mathrm{kOe}$ to $4 \mathrm{kOe}$. The line connecting the points are visual guides.

In a homogeneous FM magnetic material, one expects to see a cusp at $T_{\mathrm{C}}$ where the magnetic material changes its magnetic state from FM to PM. However, there is no cusp at $T_{\mathrm{C}}$ in this sample. At this temperature, $\Delta C_{p}$ has a value of $1.2(\mathrm{~J} / \mathrm{kg} \mathrm{K})$ measured at $H=4 \mathrm{kOe}$. In section 3.4.3, I explained the effect of the applied $H$ which induces considerable short-range 2D FM order above $T_{\mathrm{C}}$ which results in the presence of $M_{\mathrm{r}}>0, M_{\mathrm{S}}>0, H_{\mathrm{C}}>0$ (Figure 3.7). The presence of the short-range FM order causes the $\Delta C_{p}$ $>0$ above $T_{\mathrm{C}}$ and a broad peak around $300 \mathrm{~K}$ in figure Figure 4.4. The presence of the SPM phase in this sample changes the trend of heat capacity in the temperature range $T<T_{\mathrm{C}}$.

\subsection{Relative Cooling Power Calculation (RCP)}

To quantify the efficiency of refrigeration, the RCP (relative cooling power) is calculated. RCP is the amount of heat transfer between the cold and the hot reservoir in a complete refrigerator cycle $[4,29]$. This parameter is based on the change of the magnetic entropy as

$$
R C P(S)=-\Delta S_{M}(\max ) \Delta T_{F W H M},
$$


where $\Delta S_{M}(\max )$ is the maximum of the entropy change and $\Delta T_{F W H M}$ is the working temperature. The $\Delta T_{F W H M}$ is defined as the full width at half maximum (FWHM) of $-\Delta S_{M}$ vs. $T[4,21]$.

Since there are two peaks in the $-\Delta S_{M}$ vs. $T$ plot of Figure 4.2, both peaks are fitted to two Gaussian functions [30] centered around $220 \mathrm{~K}$ and $270 \mathrm{~K}$ using FWHM of these peaks as $\triangle T_{F W H M}$ for each case. The results from these fits are presented in Figure 4.5-a shows the RCP values of the $7.6 \mathrm{~nm} \mathrm{LSMO/STO}$ sample at $220 \mathrm{~K}$ and $270 \mathrm{~K}$. Due to magnetic inhomogeneities there are two RCPs. The maximum RPs are near $\sim 220 \mathrm{~K}$ and $\sim 270 \mathrm{~K}$ which are very close to SPM-FM and FM-PM magnetic phase transition [4]. By increasing $H$ the RCP at $220 \mathrm{~K}$ decreases while the RCP at $270 \mathrm{~K}$ increases. The effect of $H$ is not limited only to the change of RCP. Figure 4.5-b shows that with an increase in $H$, the FM volume fraction $(\beta)$ increases whereas the SPM volume fraction $(\alpha)$ decreases. This can be understood in terms of the alignment of the SPM fraction along the $H$ direction similar to the FM fraction, thereby increases the FM fraction and reducing the SPM fraction. 


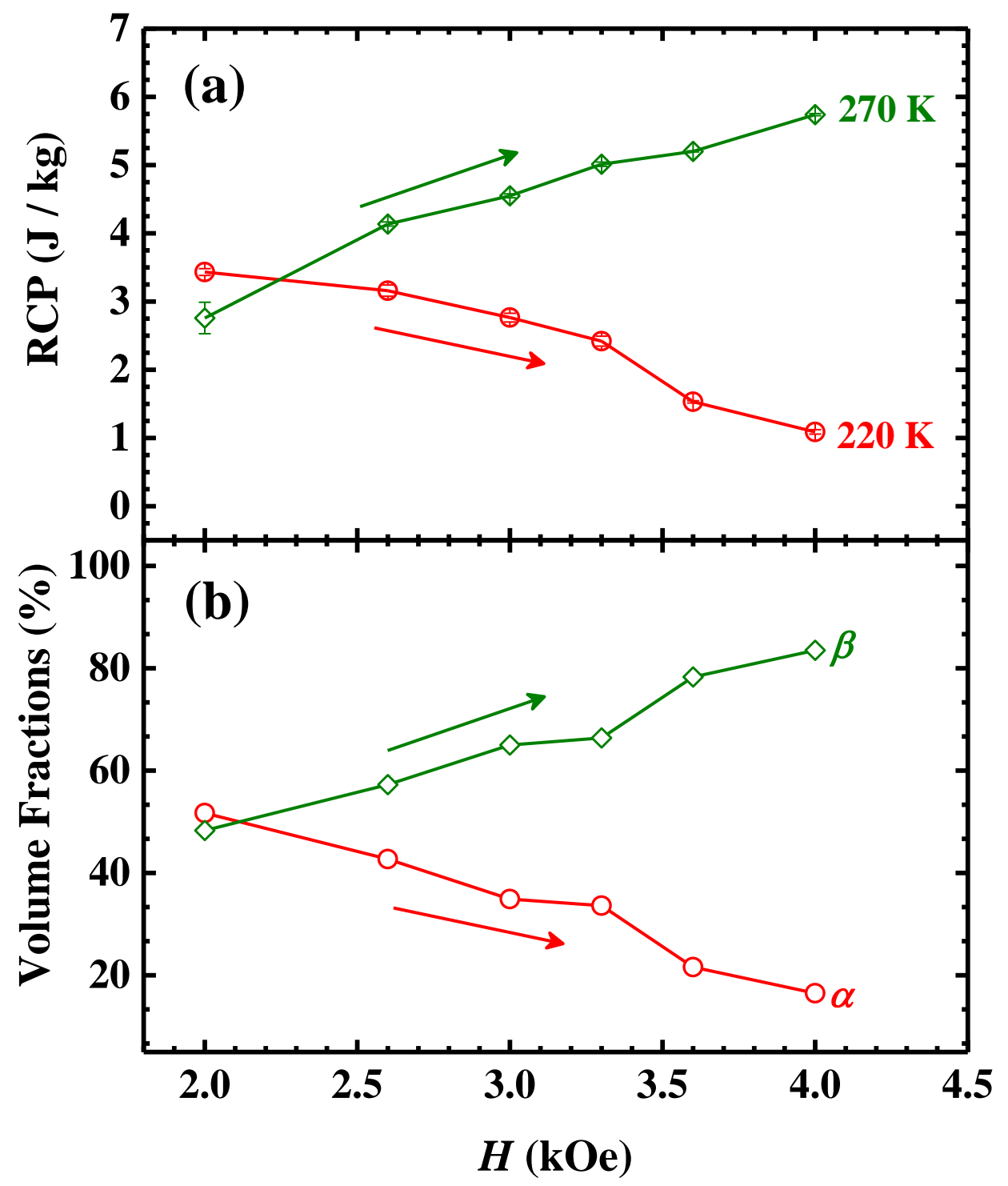

Figure 4.5. (a) The calculated relative cooling power (RCP) of the $7.6 \mathrm{~nm} \mathrm{LSMO/STO} \mathrm{sample} \mathrm{versus}$ applied magnetic field at $220 \mathrm{~K}$ and $270 \mathrm{~K}$. (b) Relative volume fractions of the SPM $(\alpha)$ and FM $(\beta)$ magnetic phases. The lines connecting the data points are visual guides.

To compare the RCP values of this sample with those reported in the literature on different samples of LSMO, I list these numbers in Table 4-1. According to the table, LSMO NPs and films with similar particle size and film thicknesses are having close RCP values within the range of 40-66 ( $\left.\mathrm{J} \mathrm{kg}^{-1}\right)$ [4,7,31,32]. However, the RCP of the $7.6 \mathrm{~nm}$ LSMO/STO film is comparatively small, likely due to several reasons. First, this film is a soft FM material since its magnetization is saturated at smaller $H=4 \mathrm{kOe}$ compared to the saturation $H=10 \mathrm{kOe}$ for other LSMO NPs and films. Second, the thickness of this film is smaller than the diameter of NPs and thickness of other LSMO films listed in Table 1. The thickness effect on $M_{\mathrm{S}}$ is 
discussed in section 1.4. As shown in Figure 1.8 in by decreasing the thickness the $M_{\mathrm{S}}$ decreases, and this small thickness lowers the saturation magnetization $M_{\mathrm{S}}$ and the finite size effect is evident. Third, the presence of $\mathrm{MI}$ in this sample also lowers $M_{\mathrm{S}}$ a and $-\Delta S_{M}$ which in turn lowers the RCP.

Table 4-1. Summary of the reported RCPs of LSMO systems compared with the data on the $7.6 \mathrm{~nm} \mathrm{LSMO} / \mathrm{STO}$ film.

\begin{tabular}{|c|c|c|c|c|}
\hline Composition & $H(\mathrm{kOe})$ & $T(\mathbf{K})$ & $\operatorname{RCP}\left(\mathrm{J} \mathrm{kg}^{-1}\right)$ & Reference \\
\hline $\mathrm{La}_{0.67} \mathrm{Sr}_{0.33} \mathrm{MnO}_{3} / \mathrm{SrTiO}_{3}(20 \mathrm{~nm}$ film $)$ & 15 & 312 & 50.6 & [82] \\
\hline $\begin{array}{l}\quad \mathrm{La}_{0.67} \mathrm{Sr}_{0.33} \mathrm{MnO}_{3} / \mathrm{La}_{0.3} \mathrm{Sr}_{0.7} \mathrm{Al}_{0.65} \mathrm{Ta}_{0.35} \mathrm{O}_{9} \\
(20 \mathrm{~nm} \text { film })\end{array}$ & 15 & 321 & 34.24 & [82] \\
\hline $\mathrm{La}_{0.81} \mathrm{Sr}_{0.19} \mathrm{MnO}_{3}(25 \mathrm{~nm} \mathrm{NPs})$ & 10 & 284 & 66.5 & [91] \\
\hline $\mathrm{La}_{0.83} \mathrm{Sr}_{0.17} \mathrm{MnO}_{3}(25 \mathrm{~nm} \mathrm{NPs})$ & 10 & 327 & 49 & [91] \\
\hline $\mathrm{La}_{0.89} \mathrm{Sr}_{0.11} \mathrm{MnO}_{3}(27 \mathrm{~nm} \mathrm{NPs})$ & 10 & 297 & 41.4 & [91] \\
\hline $\mathrm{La}_{0.67} \mathrm{Sr}_{0.33} \mathrm{MnO}_{3}(30 \mathrm{~nm} \mathrm{NPs})$ & 10 & 361 & 41 & [92] \\
\hline $\mathrm{La}_{0.67} \mathrm{Sr}_{0.33} \mathrm{MnO}_{3}(20 \mathrm{~nm} \mathrm{NPs})$ & 10 & 370 & 40 & [81] \\
\hline $\mathrm{La}_{0.81} \mathrm{Sr}_{0.19} \mathrm{MnO}_{3}(25 \mathrm{~nm} \mathrm{NPs})$ & 4 & 284 & 24 & [91] \\
\hline $\mathrm{La}_{0.83} \mathrm{Sr}_{0.17} \mathrm{MnO}_{3}(25 \mathrm{~nm} \mathrm{NPs})$ & 4 & 327 & 20 & [91] \\
\hline $\mathrm{La}_{0.89} \mathrm{Sr}_{0.11} \mathrm{MnO}_{3}(27 \mathrm{~nm} \mathrm{NPs})$ & 4 & 297 & 15.7 & [91] \\
\hline $\mathrm{La}_{0.67} \mathrm{Sr}_{0.33} \mathrm{MnO}_{3}(30 \mathrm{~nm} \mathrm{NPs})$ & 4 & 361 & 14.6 & [92] \\
\hline $\mathrm{La}_{0.67} \mathrm{Sr}_{0.33} \mathrm{MnO}_{3}(20 \mathrm{~nm} \mathrm{NPs})$ & 4 & 370 & 14 & [81] \\
\hline $\mathrm{La}_{0.7} \mathrm{Sr}_{0.3} \mathrm{MnO}_{3} / \mathrm{SrTiO}_{3}(7.6 \mathrm{~nm}$ thin film $)$ & 4 & 270 & 6.0 & This work \\
\hline
\end{tabular}

If we approximate the two peaks in the $-\Delta S_{M} v s . T$ by a single broad peak as shown in Figure 4.6, the FWHM $\left(\Delta T_{F W H M}\right)$ and height $\left(-\Delta S_{M}(\max )\right)$ of the curve shown in this figure are $\sim 80 \mathrm{~K}$, and $\sim 0.16$ $\mathrm{J} / \mathrm{kgK}$ respectively. Consequently, the $\mathrm{RCP}$ of this sample would be $\sim 13 \mathrm{~J} / \mathrm{kg}$ which is comparable with 
reported RCPs of the other LSMO samples listed in Table 4-1. This exercise shows that MI is a detriment to achieving higher RCP.

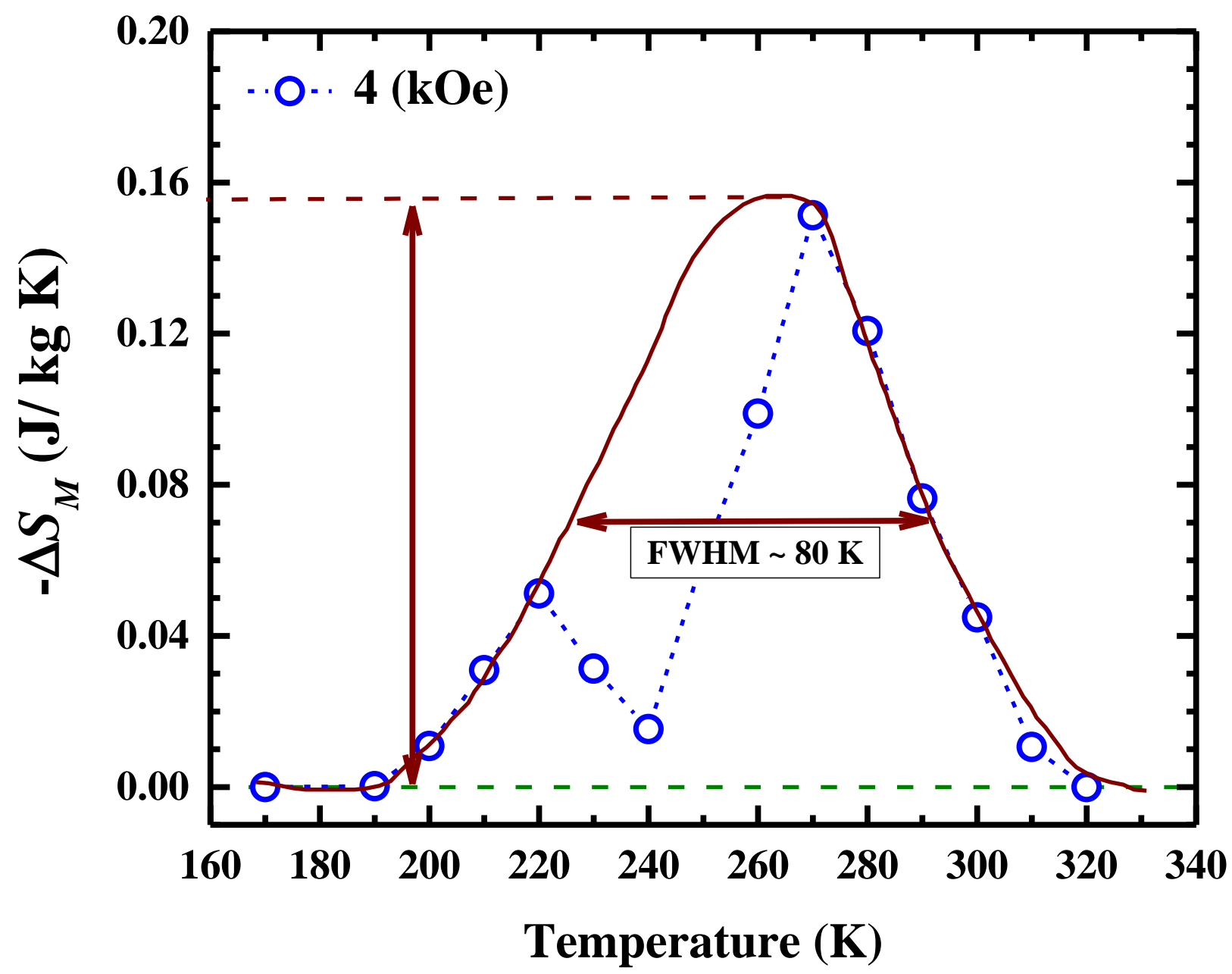

Figure 4.6. Magnetic entropy of the sample versus temperature at $H=4 \mathrm{kOe}$. The blue circles are the data and the dashed line connecting the data points is for the visual guide. The solid brown line is the connected curve of the two peaks at $220 \mathrm{~K}$ and $270 \mathrm{~K}$. (For interpretation of the references to color in this figure legend, the reader is referred to the Web version of this article.)

\subsection{Conclusion}

In this chapter the analysis of the MC properties of the $7.6 \mathrm{~nm}, \mathrm{LSMO} / \mathrm{STO}$ thin film has been reported. The notable results include the determination of $T_{\mathrm{C}}$, the nature of magnetic phase transitions at $T_{\mathrm{B}}=220 \mathrm{~K}$ and $T_{\mathrm{C}}=270 \mathrm{~K}$, and the determination of the volume ratio of each magnetic phase. The results of in $-\Delta S_{\mathrm{M}}$ vs. $T$ showed the presence of two peaks which each was related to SPM-FM and FM-PM magnetic phase transitions, presented in Figure 4.2. By fitting these peaks to two Gaussian functions, computed areas under 
the two peaks are used to estimate the volume fractions of the SPM/FM phases. The volume fractions are $17 \% / 83 \%$ for SPM and FM phases, respectively which are close to volume calculations from the dead layer model from saturation magnetization at $H=4 \mathrm{kOe}$ (sections 3.4.1 and 4.3).

here shows the presence of magnetic inhomogeneity in the film since two broad peaks are observed in the magnetic entropy vs. $T$ data. The field variation of $\Delta S_{\mathrm{M}}$ was analyzed by fitting the graphs to the exponent power law $\Delta S_{\mathrm{M}}=a H^{n}$ (Figure 4.3) which the ' $n$ ' exponent helped to certify the results of chapter 3 on the magnetic phase transitions at $T_{\mathrm{B}}$ and $T_{\mathrm{C}}$.

The RCP of the $7.6 \mathrm{~nm} \mathrm{LSMO/STO} \mathrm{thin} \mathrm{film} \mathrm{is} \mathrm{also} \mathrm{evaluated} \mathrm{and} \mathrm{compared} \mathrm{with} \mathrm{those} \mathrm{reported} \mathrm{in} \mathrm{the}$ literature on other samples of the LSMO system. The comparatively smaller magnitude of the RCP of the $7.6 \mathrm{~nm} \mathrm{LSMO/STO}$ thin film is attributed to the presence of magnetic inhomogeneity present in the film and the nano-size effects related to the small thickness of the film. The effect of applied $H$ on the volume fractions of the FM $(\beta)$ and SPM $(\alpha)$ phases shows an increase (a decrease) in the FM (SPM) fractions with the increase in $H$ due to alignment of the magnetization with the increase in $H$ as shown in Figure 4.5. 


\section{Chapter 5 \\ Temperature and Frequency Dependence of AC Susceptibility}

\subsection{Introduction}

In this chapter, I discuss the ac magnetic susceptibility measurements on a $7.6 \mathrm{~nm}$ thin film of LSMO/STO. In previous chapters (3\&4) the two magnetic phases such as SPM and FM were detected by studying the temperature and field variation of magnetization as well as the temperature change of magnetic entropy. The goal of this chapter is to understand the strength of the magnetic interaction between the magnetic SPM and FM magnetic phases and spin dynamics of this sample. The theoretical discussions on ac magnetic susceptibilities are discussed here and further details of ac magnetic susceptibility measurements are discussed in subsequent sections of this chapter. The magnetization of the sample was measured using an ac measurement system (ACMS) for the Physical Property Measurement System (PPMS) purchased from Quatum Design, Inc. The experimental data were taken with the help of collaborators in this project. The results of this chapter have been published by the author recently $(\mathrm{N}$. Mottaghi, et al, J. Appl. Phys. 128, 073903 (2020)) and presented at the 65th Annual Conference on Magnetism and Magnetic Materials (MMM 2020, Virtual Conference).

\subsection{Theoretical Background}

\subsubsection{Temperature and Frequency-Dependent on AC susceptibilities}

AC magnetic susceptibility is a useful technique to study the time dynamic effects of a magnetic system. In previous sections the applied magnetic field was static and the magnetic equilibrium states were considered. In this section, we assume the applied magnetic field is a function of time as $H(t)=H_{0} e^{i \omega t}$, where $\omega$ is $2 \pi f_{m}$ and $f_{m}(\mathrm{~Hz})$ is the frequency of the oscillations.

The ac susceptibility data and analysis will be presented in chapter 5. In this section, I cover the basic equations used in this analysis. As discussed in chapter 1, the relation between magnetization and the magnetic field is presented by the equation-(1-3). In AC measurement the magnetic field varies with time and this equation is replaced with

$$
M=\chi H_{0} e^{i \omega t}
$$

where $H_{0}$ is the ac field amplitude, $\chi$ is the ac magnetic susceptibility, $\omega=2 \pi f_{m}$ and $f_{m}(\mathrm{~Hz})$ is the frequency of the ac magnetic field.

To find the $\chi$, we can borrow the equation of motion of a damped harmonic oscillator, which is a good approximation. The equation of motion is [93] 


$$
m \ddot{x}+\alpha \dot{x}+k x=f,
$$

where $m$ is the mass, $\alpha$ is the damping constant, and $k$ is the spring constant. The resonant frequency of the oscillator is $\omega_{0}^{2}=k / m$, with the damping term $\gamma=\alpha / m$. If we remove the inertial term $(m \sim 0)$ in the equation-(5-2) and solutions for $\chi(\omega)=\chi(\omega) f(\omega) e^{-i \omega t}$

$$
\chi(\omega)\left(-i e^{-i \omega t} \omega \frac{\alpha}{k}+e^{-i \omega t}\right)=\frac{1}{k}
$$

and by simplifying this equation

$$
\chi(\omega)=\frac{\frac{1}{k} e^{-i \omega t}}{1-i \omega \tau}
$$

where $\tau=\alpha / k$. Equation-(5-4) in the limits of $t<<0$ it becomes

$$
\chi(\omega)=\frac{\frac{1}{k}}{1-i \omega \tau} .
$$

In the harmonic oscillator solution, it is common to include an adiabatic response, $\chi_{s}=\chi(\infty)$,

$$
\chi(\omega)=\chi_{s}+\frac{\frac{1}{k}}{1-i \omega \tau},
$$

and by replacing the $1 / k=\chi_{T}-\chi_{s}$ and considering $\chi(\omega)=\chi^{\prime}(\omega)+i \chi^{\prime \prime}(\omega)$

$$
\begin{aligned}
& \chi(\omega)=\chi_{s}+\frac{\chi_{T}-\chi_{s}}{1-i \omega \tau}, \\
& \chi^{\prime}(\omega)=\chi_{s}+\frac{\chi_{T}-\chi_{s}}{1+(\omega \tau)^{2}}, \\
& \chi^{\prime \prime}(\omega)=\frac{\chi_{T}-\chi_{s}}{1+(\omega \tau)^{2}} \omega \tau,
\end{aligned}
$$

where $\chi_{T}=\chi(0)$ is the isothermal susceptibility.

If there is not a single $\tau$ and there is a distribution of relaxation times, a simple empirical law can present the ac magnetic susceptibility as

$$
\chi(\omega)=\chi_{s}+\frac{\chi_{0}-\chi_{s}}{1+(i \omega \tau)^{1-\alpha}}
$$


and

$$
\begin{aligned}
& \chi^{\prime}(\omega)=\chi_{s}+\frac{\left(\chi_{0}-\chi_{s}\right)\left[1+(\omega \tau)^{1-\alpha} \sin \left(\frac{\alpha \pi}{2}\right)\right]}{1+2(\omega \tau)^{1-\alpha} \sin \left(\frac{\alpha \pi}{2}\right)+(\omega \tau)^{2-2 \alpha}}, \\
& \chi^{\prime \prime}(\omega)=\frac{\left(\chi_{0}-\chi_{s}\right)(\omega \tau)^{1-\alpha} \cos (\alpha \pi / 2)}{1+2(\omega \tau)^{1-\alpha} \sin \left(\frac{\alpha \pi}{2}\right)+(\omega \tau)^{2-2 \alpha}},
\end{aligned}
$$

which the larger value of $\alpha$ presents the wider distribution in relaxation [94].

By combining equations-(5-9) the relation between $\chi^{\prime \prime}(\omega)$ and $\chi^{\prime}(\omega)$ is as follows:

$$
\chi^{\prime \prime}\left(\chi^{\prime}\right)=-\frac{\chi_{0}-\chi_{s}}{2 \tan \left[(1-\alpha) \frac{\pi}{2}\right]}+\left\{\left(\chi^{\prime}-\chi_{s}\right)\left(\chi_{0}-\chi^{\prime}\right)+\frac{\left(\chi_{0}-\chi_{s}\right)^{2}}{4 \tan ^{2}\left[(1-\alpha) \frac{\pi}{2}\right]}\right\}^{\frac{1}{2}} .
$$

By plotting $\chi^{\prime \prime}(\omega)$ vs. $\chi^{\prime}(\omega)$, we can plot Cole-Cole plots at a fixed temperature and fitting into the equation- the important parameters such as $\chi_{0}$ and $\alpha$ is calculated. Knowing the calculated values and insert them back into equations-(5-9) the relaxation time at each temperature is calculated.

\subsection{Investigation of Temperature and Frequency Dependence of AC Susceptibilities}

Measurements of the ac susceptibilities were done by using an ac measurement system attached to the commercial physical property measurement system (PPMS from Quantum Design Inc.). The temperature range was chosen for $170 \mathrm{~K}$ to $310 \mathrm{~K}$ to cover both SPM to FM and FM to PM magnetic phase transitions at $T_{\mathrm{B}}$ and $T_{\mathrm{C}}$ of the sample, respectively by using frequencies $f_{\mathrm{m}}=0.1-10 \mathrm{kHz}$. In magnetic measurements, the applied magnetic field was parallel to the plane of the film, thus eliminating the need for corrections due to the demagnetization field. To measure the ac magnetic susceptibilities $\chi^{\prime}$ and $\chi^{\prime \prime}$, the coils of the PPMS were first demagnetized at room temperature followed by cooling the sample to $170 \mathrm{~K}$ in the dc field $H=50$ Oe. After the temperature became stable, the $H$ was switched off to zero, and measurements of $\chi^{\prime}$ and $\chi^{\prime \prime}$ were done with increasing temperatures up to $310 \mathrm{~K}$ at different $f_{\mathrm{m}}=0.1,1,5$, and $10 \mathrm{kHz}$ using $H_{\mathrm{ac}}=10$ Oe for two cases of superposed bias dc fields: (i) $H=0$ and (ii) $H=100$ Oe.

The plots of $\chi^{\prime}$ and $\chi^{\prime \prime}$ vs $T$ from $170 \mathrm{~K}$ to $310 \mathrm{~K}$ are shown in Figure 5.1 (a) for the case of $H=0$ with the expanded view of the plots near $T=270 \mathrm{~K}$ shown in Figure 5.1 (b). The expanded view of the region near $T=230 \mathrm{~K}$ in $\chi^{\prime}$ vs $T$ plot shows a broad peak whose frequency dependence is difficult to determine because of the broadness of the peak. In chapter 3, section 3.4.2, and Figure 3.3, this peak was also determined by the bifurcation between zero-field-cooled (ZFC) and field-cooled (FC) data that was 
interpreted as the SPM phase transition temperature. The observation of a broad peak near $230 \mathrm{~K}$ is a confirmation of associating the peak with $T_{\mathrm{B}}$ of nanoclusters. The second peak in $\chi^{\prime}$ and $\chi^{\prime \prime}$ vs $T$ plots are around $T=270 \mathrm{~K}$ in Figure 5.1 (b). The peaks at $T_{\mathrm{B}}$ and $T=270 \mathrm{~K}$ shifts to higher $T$ and also the magnitude of the peaks decrease with an increase in measuring frequency from $0.1 \mathrm{kHz}$ to $10 \mathrm{kHz}$.
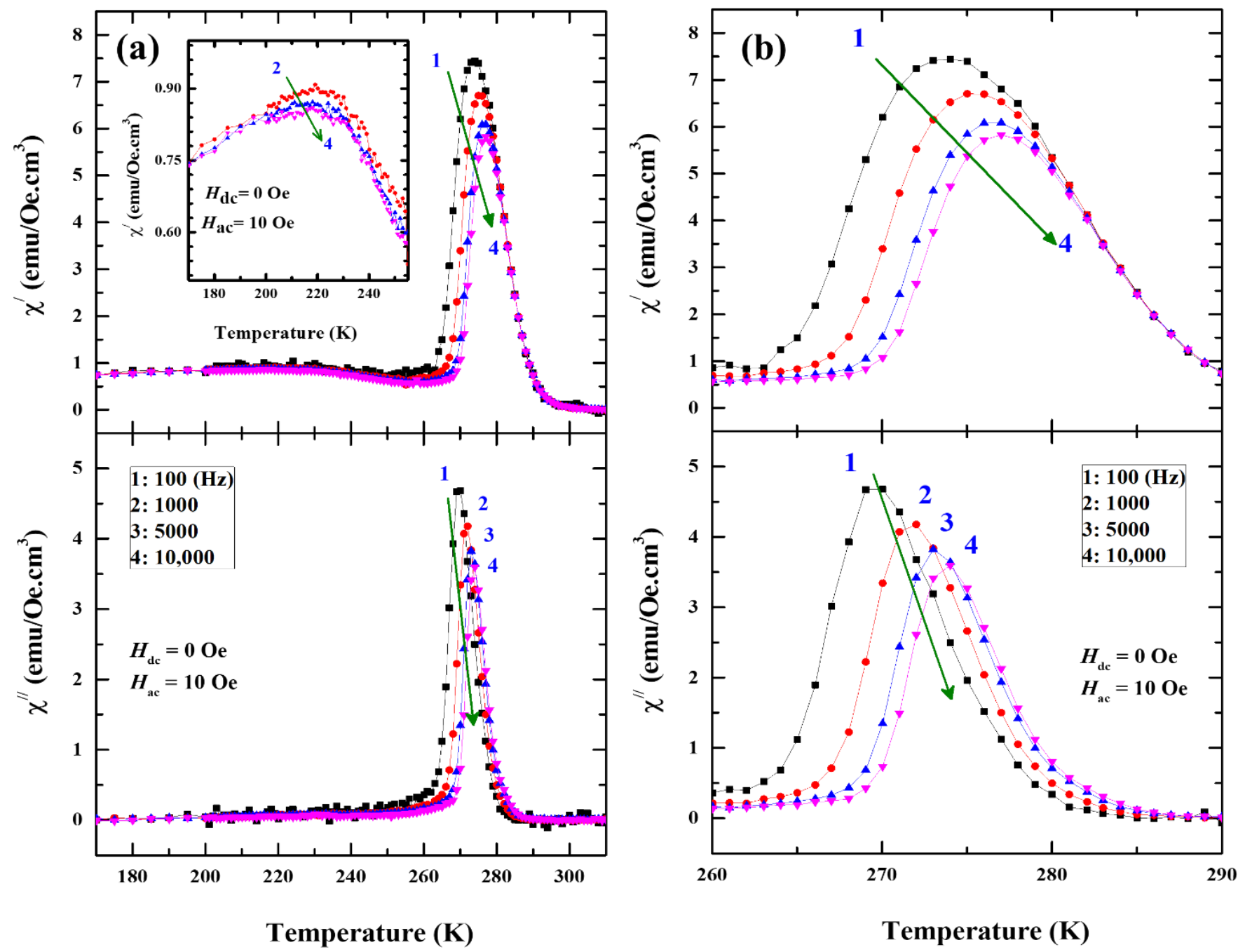

Figure 5.1. (a) Plots of experimental $\chi^{\prime}$ and $\chi^{\prime \prime}$ vs temperature at four frequencies with the inset in the top figure showing an expanded view of the broad peak near $230 \mathrm{~K}$. (b) High-temperature zoom of the $\chi^{\prime}$ and $\chi^{\prime \prime}$ vs temperature data at different frequencies [76].

To understand the nature of the peak at $T=270$, I can explain it in determining the $T_{\mathrm{C}}$ from the temperature dependence of magnetization ( $M$ vs $T$ ) in a ferromagnet, like LSMO, which depends on the applied dc magnetic field $H$. To understand the effect of $H$ on $T_{\mathrm{C}}$ of the sample the $T_{\mathrm{C}}$ was calculated at different applied fields from the plots of $\mathrm{d} M / \mathrm{d} T$ vs $T$ determined from Figure 3.3 in section 3.4.2 for $H=$ 
50, 100, 200, 500, and 1000 Oe. The minimum in $\mathrm{d} M / \mathrm{d} T$ vs $T$ is defined as $T_{\mathrm{C}}$ (Figure 5.2-a), which increases with an increase in $H$. The plot of $T_{\mathrm{C}}$ vs $H$ is shown in Figure 5.2-b and the solid line is fit to

$$
T_{\mathrm{C}}(H)=A H^{n}+T_{\mathrm{C}}(0)
$$

where $n$ is a constant determined from the fit and $T_{\mathrm{C}}(0)$ is the value of the $T_{\mathrm{C}}$ in the absence of $H$. The fit shown in Figure 5.2-b yields the parameters $T_{\mathrm{C}}(0)=270 \mathrm{~K}, n=0.15$, and $A=9.74 \mathrm{~K} / \mathrm{Oe}^{\mathrm{n}}$. The obtained value of $T_{\mathrm{C}}(0)$ at $T=270 \mathrm{~K}$ is the exact peak position of the second peak in $\chi^{\prime}$ and $\chi^{\prime \prime}$ vs $T$ plots in Figure 5.1 therefore the peak at $T=270 \mathrm{~K}$ is the $T_{\mathrm{C}}$.
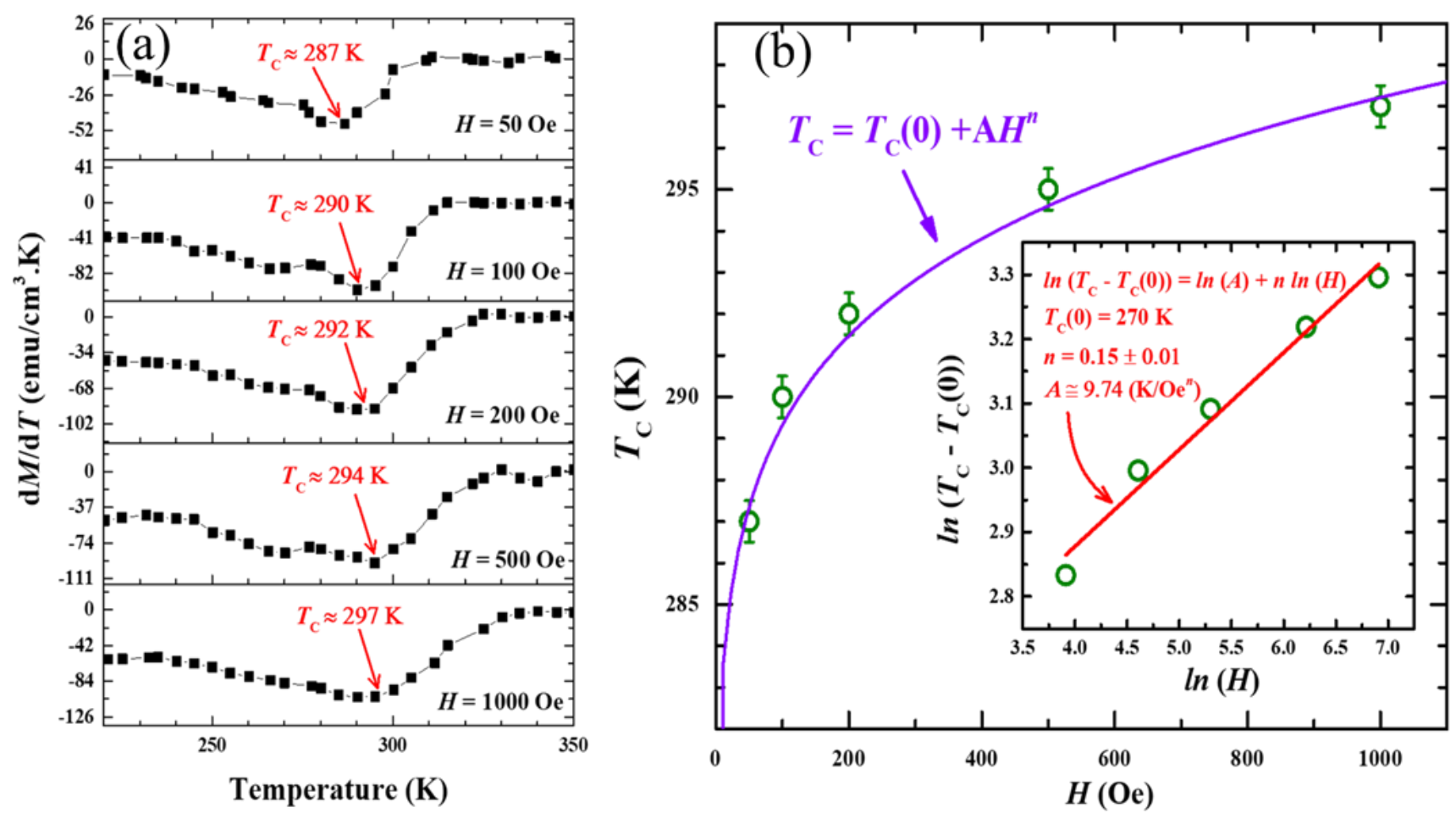

Figure 5.2. (a) Plots of computed $\mathrm{d} M / \mathrm{d} T$ vs $T$ for different $H$ to determine $T_{\mathrm{C}}$ using the $M$ vs $T$ data of Figure 3.3 in section 3.4.2. (b) Magnetic field variation of $T_{\mathrm{C}}$ with the solid purple line fit to the equation5-11) and the inset showing the plot of $\ln \left[T_{\mathrm{C}}(H)-T_{\mathrm{C}}(0)\right]$ vs $\ln H$ with the red line as the linear fit [76].

The theoretical basis for equation-5-11 is established using Landau theory of phase transition according to the free energy $F$ near $T_{\mathrm{C}}$ in a magnetic field $H$ can be written in terms of the order parameter $M$ as [95]

$$
F=\frac{1}{2} a(T) M^{2}+\frac{1}{4} b(T) M^{4}+\frac{1}{6} c(T) M^{6}-M H,
$$

with minimizing $F$ with respect to $M$ yields 


$$
H=a(T) M+b(T) M^{3}+c(T) M^{3}
$$

Where the coefficient $a(T)=\left(T_{\mathrm{C}}-T\right) / T_{\mathrm{C}}$ is often used and from the Takahashi theory for itinerant ferromagnets, $b(T)=0$ at $T=T_{\mathrm{C}}$ [95]. These substitutions yield $H=c(T) M^{5}$ at $T_{\mathrm{C}}$, which implies that applied $H$ increases $M$ at $T_{\mathrm{C}}$ with $M \sim H^{1 / 5}$ and so $n=0.2$. This is close to $n=0.15$ determined from the fit of the data to equation-(5-11).

The effect of dc applied field $\left(H_{\mathrm{dc}}\right)$ on $T_{\mathrm{C}}$ is discussed above via dc bulk magnetometry measurements. This effect also is studied by ac bulk magnetometry measurements which the measurement protocol as discussed above. The effect of applied dc field of $H=100$ Oe on the peaks of $\chi^{\prime}$ and $\chi^{\prime \prime}$ in $7.6 \mathrm{~nm}$ LSMO/STO film near $270 \mathrm{~K}$ is shown in Figure 5.3. The peak in $\chi^{\prime}$ shifts to higher $T$ by about $\Delta T \approx 15 \mathrm{~K}$ and its magnitude is reduced by a factor of about 5 , where the peak in $\chi^{\prime \prime}$ completely disappears. However, the shift in $\chi^{\prime}$ to higher temperatures in $H=100$ Oe is consistent with the increase of $T_{\mathrm{C}}$ with applied $H$ shown in Figure 5.2-(b). The observed change in intensity of both $\chi^{\prime}$ and $\chi^{\prime \prime}$ in $H=100$ Oe may be due to the stabilization of the magnetic ordering with applied $H$, thus making $\chi^{\prime \prime}=0$. Also, it is noted that the nanoscale thickness of the LSMO film investigated here may be the reason for the observation of the frequency-dependent peaks near $T_{\mathrm{C}}$. Further insights into this phenomenon may come from similar ac measurements on films with different thicknesses. 


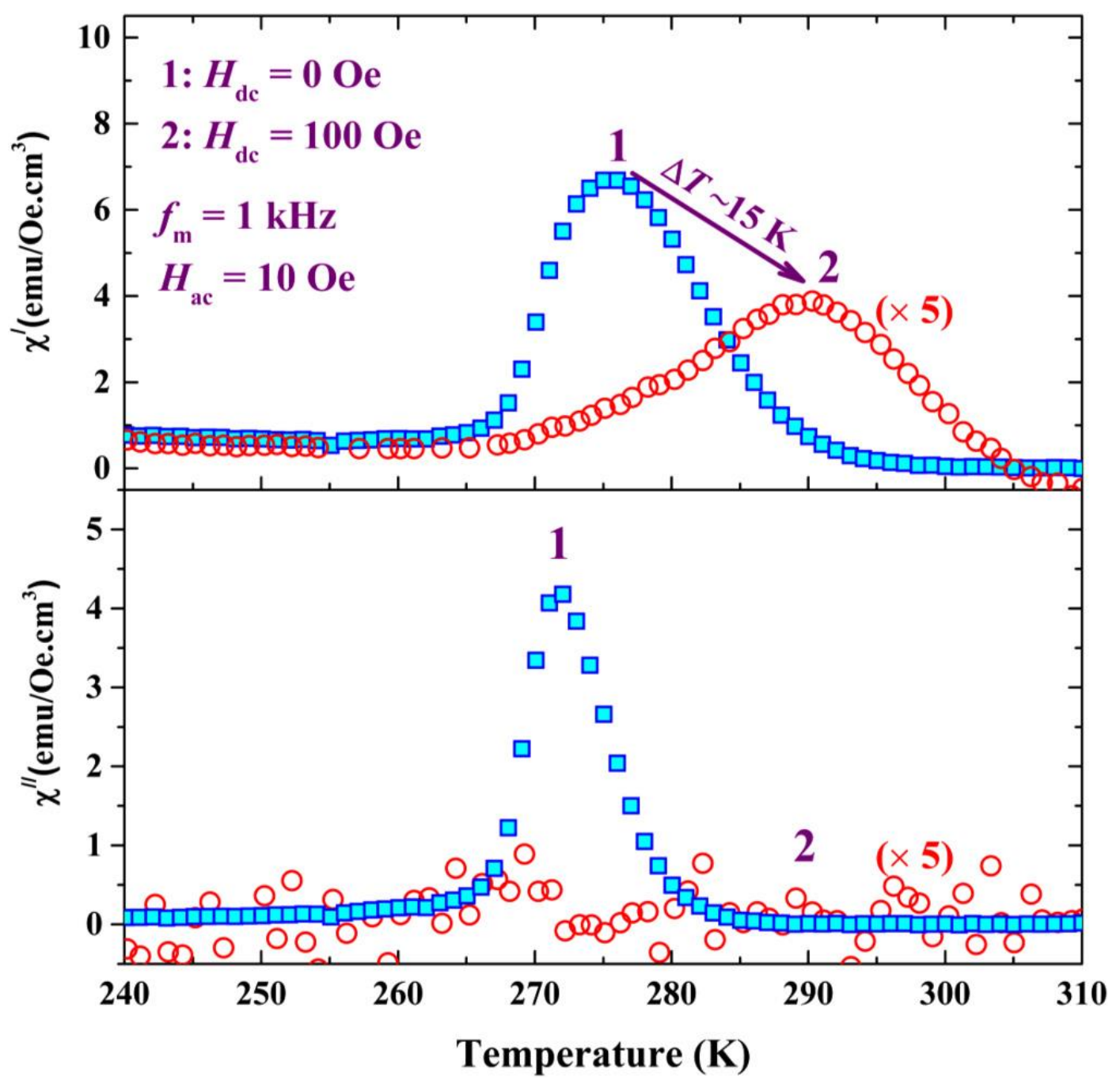

Figure 5.3. Plots of experimental $\chi^{\prime}$ and $\chi^{\prime \prime}$ vs temperature for one frequency $f=1 \mathrm{kHz}$ and $H(\mathrm{ac})=$ 10 Oe but for two static fields $H=0$ Oe (blue solid squares) and $H=100 \mathrm{Oe}$ (red open circles). The data of $\chi^{\prime}$ and $\chi^{\prime \prime}$ for $H=100$ Oe are multiplied by a factor of 5 for visual clarity [76]. 
To gain further information into the nature of the peak near $270 \mathrm{~K}$, its frequency dependence is analyzed by the parameter $\phi=\Delta T_{P} /\left[T_{P} \Delta \log _{10} f_{m}\right]$, where $\Delta T_{P}$ is the shift in $T_{P}$ with a change in frequency from $f_{\mathrm{m}}$ $(1)=0.1 \mathrm{kHz}$ and $f_{\mathrm{m}}(2)=10 \mathrm{kHz}, \phi=0.002$ is determined in this sample. This analysis used in different magnetic systems such as spin glasses, and non-interacting nanoparticles. For negligible interparticle interaction (IPI), $\phi \geq 0.13$ whereas for spin-glasses $\phi<0.005$. In LSMO NPs, the reported $\phi=0.03$ [96] and 0.011 [97]. The observed $\phi=0.002$ here is an order of magnitude smaller than these values of $\phi$ suggesting that the transition near $T_{\mathrm{C}}$ is due to even stronger IPI, typical of a normal second order transition but with some modifications due to the nanoscale thickness of the film. More details on this emerge from the Cole-Cole analysis of $\chi^{\prime}$ and $\chi^{\prime \prime}$ vs $T$ given below.

\subsubsection{Cole-Cole plots and Vogel-Fulcher law analysis}

To gain further information from the ac susceptibility data of Figure 5.1, the values of $\chi^{\prime}$ and $\chi^{\prime \prime}$ were determined at different temperatures in the vicinity of $T_{\mathrm{C}}$ for all frequencies using these plots. The ColeCole plot is the variation of $\chi^{\prime \prime}$ vs $\chi^{\prime}$ at various temperatures, which for the LSMO film are shown in Figure 5.4. The solid red lines are the fits to Cole-Cole equations (equation-(5-10)) for the complex susceptibility $\chi(\omega)=\chi^{\prime}(\omega)-i \chi^{\prime \prime}(\omega)$ to find the value of relaxation time $(\tau)$ at each temperature.

The temperature variation of calculated values of $\tau$ is shown in Figure 5.5-(b). The data fit well to the Vogel-Fulcher law (equation-(3-47)) which reveals the interparticle interaction. The fit yields $T_{0}=245 \mathrm{~K}$ which the $\tau_{0}=1.2 \times 10^{-9}(\mathrm{~s})$ and $\Delta E / k_{\mathrm{B}}=270 \mathrm{~K}$. The $\Delta E / k_{\mathrm{B}}$ value is equal to $T_{\mathrm{C}}(0)=270 \mathrm{~K}$ of the sample and the $T_{0}$ value lies in-between $T_{\mathrm{B}}$ and $T_{\mathrm{C}}(0)$. It is worth noting that in the region between $T_{\mathrm{B}}<T<T_{\mathrm{C}}(0)$ the negative magnetization relaxation occurs (Figure 3.9) which is the signature of the magnetic inhomogeneities and magnetic phase competition between the SPM and FM magnetic phases. The detailed discussion on the role of the SPM and FM phases is observed through studying the $M$ vs $T$ and hysteresis loops in sections 3.4.2 and 3.4.3 where the negative magnetization and hysteresis loop inversion occurs. 

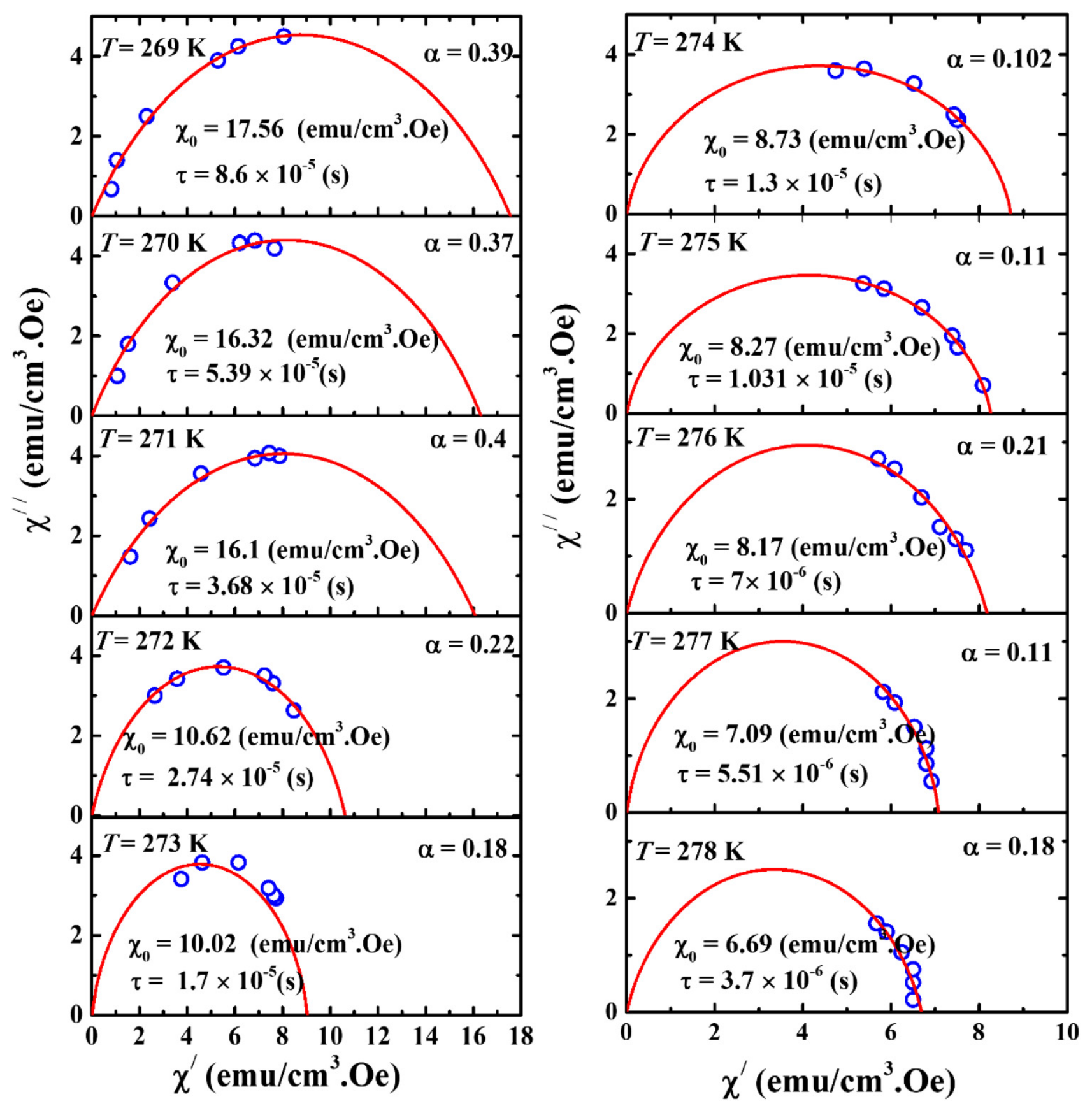

Figure 5.4. The Cole-Cole plots at different temperatures from $269 \mathrm{~K}$ to $278 \mathrm{~K}$. The solid red curves are fit to the equation-(5-10) with the parameters of the fits listed in each figure [76]. 

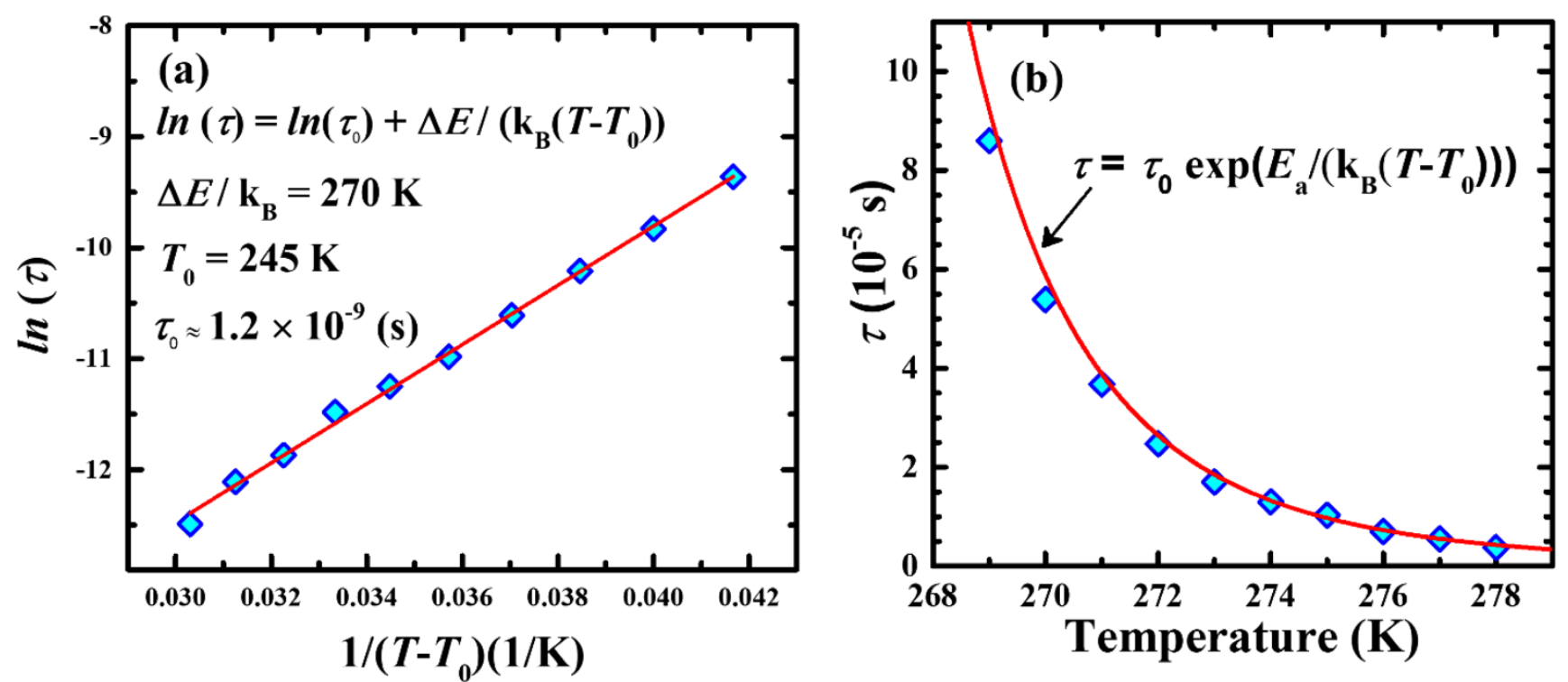

Figure 5.5. (a) The best-fit linear line in the plot of $\ln (\tau)$ vs $1 /\left(T-T_{0}\right)$ based on the Vogel-Fulcher law [equation-Error! Reference source not found.(3-47)] to determine the $\Delta E / k_{\mathrm{B}}$ and $\tau_{0}$. (b) Shows how the calculated values of $\tau$ from the fit the Vogel-Fulcher law using the parameters determined from the plot in (a) [76].

\subsection{Conclusions}

In this chapter, I presented the ac magnetic susceptibility measurements on a $7.6 \mathrm{~nm}$ LSMO/STO sample and the results and analysis of these measurements. From the plots of $\chi^{\prime}$ and $\chi^{\prime \prime}$ vs $T$ the two peaks at $T=$ $T_{\mathrm{B}}=230 \mathrm{~K}$ and $T=T_{\mathrm{C}}=270 \mathrm{~K}$ corresponding to SPM and FM phase transitions are visible. The presence of the two peaks is the confirmation of the presence of double peaks temperature dependence of the change of magnetic entropy in section 4.2 from Chapter 4 . The relaxation time $\tau$ has been calculated at different temperatures via Cole-Cole analysis $\left(\chi^{\prime \prime}\right.$ vs $\chi^{\prime}$ ) whose temperature dependence is shown to fit the VogelFulcher law. The fit to Vogel-Fulcher law yields $\tau_{0}=1.2 \times 10^{-9}(\mathrm{~s}), T_{0}=245 \mathrm{~K}$ and $\Delta E / k_{\mathrm{B}}=270 \mathrm{~K}$. The closeness of $T_{0}$ to $T_{\mathrm{B}} \sim 230 \mathrm{~K}$ of the SPM phase of the nanoclusters present in the surface layer and $\Delta E$ / $k_{\mathrm{B}}$ $=T_{\mathrm{C}}(0)$ infers strong magnetic coupling of the SPM and FM phases. As I discussed in chapters Chapter 3Chapter 4, the strong magnetic coupling between SPM and FM is the source of negative magnetization and hysteresis loop inversion. 


\section{Chapter 6 Conclusions and Publications}

\subsection{Conclusions}

The objective goals of this dissertation are to study the magnetic dead layers properties in LSMO/STO thin films which in particular the sample $7.6 \mathrm{~nm}$ is selected and the magnetic properties are studied in detail. To understand the dead layer effect in the LSMO system, first, I used the reported results of published literature and fit the dead layer model to calculate the dead layer thickness for LSMO films grown at different oxygen pressures with different thicknesses (section 1.4). The thickness of the dead layer increases as the oxygen $\left(\mathrm{O}_{2}\right)$ pressure during the growth decreases which implies by reducing the $\mathrm{O}_{2}$ pressure the double-exchange bounds breaks down which results in reducing the FM interaction between the manganese cations.

The analysis of reported results on LSMO thin films gave me a general knowledge to study the magnetic properties of this complex oxide film in detail. Therefore, the detailed study of the ac and dc magnetic measurements of a $7.6 \mathrm{~nm} \mathrm{LSMO/STO} \mathrm{sample} \mathrm{are} \mathrm{presented} \mathrm{in} \mathrm{this} \mathrm{dissertation.} \mathrm{Other} \mathrm{magnetic} \mathrm{samples}$ such as the different thickness of LSMO/STO have been studied The samples were fabricated by pulsed laser deposition and the growth was monitored by in situ reflections high energy electron diffraction (section 2.2.2) which in particular the $7.6 \mathrm{~nm}$ is presented. The structural properties of the samples have been characterized using X-ray diffraction (XRD) and X-ray reflectivity (XRR).

The magnetic properties of this sample were measured in the presence of $\mathrm{dc}$ and ac modes of the applied magnetic field. Temperature variation of magnetization was performed from low applied field $(H=50 \mathrm{Oe})$ to higher magnetic field $(H=1 \mathrm{kOe})$. In $H>100$ Oe the magnetization is saturated which is a normal behavior in ferromagnetic (FM) samples but in $H<100$ Oe there is a bifurcation between zero-field-cooled (ZFC) and field-cooled (FC) measurements in this sample (section-3.4.2). Many of our samples have shown bifurcation between the ZFC and FC protocols and in the case of the $7.6 \mathrm{~nm}$ LSMO/STO the bifurcation, the temperature is defined as the blocking temperature $T_{\mathrm{B}}=230 \mathrm{~K}$ where magnetization reduces at $T<T_{\mathrm{B}}$ and it is negative. The $T_{\mathrm{B}}$ is attributed to the superparamagnetic (SPM) phase presented in this sample.

The field variation of magnetization ( $M$ vs. $H$ ) was studied by performing ZFC hysteresis loops (HLs) from $5 \mathrm{~K}$ to $400 \mathrm{~K}$ to understand the effect of $H$ on $M$ at different temperatures (section-3.4.3). The magnetic phase competition between the SPM and FM at $T_{\mathrm{B}}<T<T_{\mathrm{C}}$ causes the inversion of HLs but for $T<T_{\mathrm{B}}$ the loops are not inverted and remanent magnetization $\left(M_{\mathrm{r}}\right)$ is $M_{\mathrm{r}}>0$ since the SPM phase is in the frozen state. The observation of inverted HLs is new in the LSMO system.

The magnetic viscosity measurements reveal that magnetic viscosity shows a peak at $T_{\mathrm{B}} \simeq 230 \mathrm{~K}$ above which $M(2 \mathrm{~h})$ becomes negative until the $T_{\mathrm{C}}$ is reached. Due to the magnetic phase competition between 
the SPM and FM phases The negative magnetic relaxation occurs between the $T_{\mathrm{B}}<T<T_{\mathrm{C}}$ of the sample (section 3.5).

To understand the nature of magnetic phase transitions, magnetocaloric studies are performed. Analysis of the MC properties of the $7.6 \mathrm{~nm}$ LSMO/STO thin film reported here shows the presence of magnetic inhomogeneity in the film since two broad peaks are observed in the magnetic entropy vs. $T$ data. The broad peak near $220 \mathrm{~K}$ is associated with the superparamagnetism (SPM) of spin clusters present in the surface layer of the film whereas the broad peak near $270 \mathrm{~K}$ is related to the transition from the FM to the PM phase. By fitting these peaks to two Gaussian functions, computed areas under the two peaks are used to estimate the volume fractions of the SPM/FM phases to be $17 \% / 83 \%$ at the saturation $H=4 \mathrm{kOe}$ (section4.3). Within $2 \%$, this result agrees with dead layer thickness calculation saturation magnetization $\left(M_{\mathrm{S}}\right)$ calculations (section-3.4.1). The results of magnetocaloric support the analysis of thickness calculation of dead layer by studying the saturation magnetization also identifies the SPM-FM and FM-PM.

The RCP of the $7.6 \mathrm{~nm} \mathrm{LSMO/STO} \mathrm{thin} \mathrm{film} \mathrm{is} \mathrm{also} \mathrm{evaluated} \mathrm{and} \mathrm{compared} \mathrm{with} \mathrm{those} \mathrm{reported} \mathrm{in} \mathrm{the}$ literature on other samples of the LSMO system. The comparatively smaller magnitude of the RCP of the $7.6 \mathrm{~nm} \mathrm{LSMO/STO}$ thin film is attributed to the presence of magnetic inhomogeneity present in the film and the nanosize effects related to the small thickness of the film. The effect of applied $H$ on the volume fractions of the FM (b) and SPM (a) phases shows an increase (a decrease) in the FM (SPM) fractions with the increase in $H$ due to alignment of the magnetization with the increase in $H$ (4.6Figure 4.5).

The temperature dependence of ac magnetic susceptibility measurements ( $\chi^{\prime \prime}$ and $\chi^{\prime}$ vs. $T$ ) shows a broad peak near at $T=230 \mathrm{~K}$ which is associated with the nanoclusters with the $90 \mathrm{~nm}$ width in $1.4 \mathrm{~nm}$ surface layer (5.3). The second peak is determined as the $T_{\mathrm{C}}=270 \mathrm{~K}$ at $H=0$ Oe which is also determined from the inflexion point of the temperature variation of magnetization fitting into the empirical equation: $T_{\mathrm{C}}(H)$ $=A H^{n}+T_{\mathrm{C}}(0)$ with $T_{\mathrm{C}}(0)=270 \mathrm{~K}$ obtained from Landau theory of phase transition near $T_{\mathrm{C}}$ in the presence of $H$ in terms of the order parameter $M$ (section 5.3). The validity of calculations is confirmed by fitting the Vogel-Fulcher law to temperature variation of time relaxation which the relaxations calculated from ColeCole plots (section 5.3.1). Moreover the coincidence of $T_{0}$ to $T_{\mathrm{B}}$ and $\Delta E / k_{\mathrm{B}}$ to $T_{\mathrm{C}}$ suggest a strong magnetic coupling of the SPM phase of the surface layer and the FM phase of the rest of the film.

\subsection{Future directions}

Complex oxide materials such as LSMO have widely used in multiferroic spintronic and ferroelectric/ferromagnetic devices due to their rich crystal structure and physical properties. Because of the FM characteristics of this material, they are widely used in hard drives. FM materials are used for nonvolatile data storage devices such as hard drives due to their permanent magnetization even in the absence 
of an external magnetic field. In the future, the demand for device applications will require smaller hard drives. Dimensional reduction in LSMO films is problematic since the tendency of this material to form a magnetic dead layer. The magnetic dead layers (MDLs) can be formed at the surface or interface where the FM properties are changed.

In this dissertation, the magnetic properties of dead layers are studied in a $7.6 \mathrm{~nm} \mathrm{LSMO/STO} \mathrm{film.} \mathrm{In}$ Chapter 3, it is shown that MDLs change the magnetic properties of this sample by reducing the magnetization below the blocking temperature $\left(T_{\mathrm{B}}\right)$ in temperature variation of magnetization $(M-T)$ of the sample. Further analysis of hysteresis loops shows that $T_{\mathrm{B}}$ is the magnetic phase transition of SPM to FM magnetic phases. Reduction in magnetization reduces the efficiency of the film in hard drive applications.

As it is discussed in chapter 1, LSMO is categorized in manganese oxide group with the general formula of $\mathrm{Re}_{1-\mathrm{x}} \mathrm{T}_{\mathrm{x}} \mathrm{MnO}_{3}$, in which $\mathrm{Re}$ is a trivalent rare earth element, and $\mathrm{T}$ is a divalent alkaline earth element. Reduction of magnetization is not limited to LSMO films and it is also observed in other materials such as $\mathrm{LaMnO}_{3}(\mathrm{LMO})$. The reduction of magnetization in different applied magnetic fields can be studied by running $M-T$ measurements in $\mathrm{ZFC}$ and FC protocols. In these measurements, we can identify the bifurcation temperatures $\left(T_{\text {bif }}\right)$. At $T_{\text {bif }}$ the magnetization of ZFC and FC cycles bifurcate. These temperatures might be the critical temperatures which can be the transition temperature of a magnetic phase. For example in a sample $7.6 \mathrm{~nm} \mathrm{LSMO} / \mathrm{STO}$ the bifurcation is at $230 \mathrm{~K}$ where the SPM to FM phase transition occurs (please see section 3.4.2). By studying the $T_{\text {bif }}$ in the LMO system, we can understand the nature of magnetic phase transitions to address the magnetization reduction in this magnetic system.

\subsection{Publications and Presentations of the Author Related to this Dissertation}

\subsubsection{Peer-Reviewed Journals}

1. N. Mottaghi, M.S. Seehra, J. Shi, M. Jain, M.B. Holcomb, Spin dynamics and relaxation in $7.6 \mathrm{~nm}$ thin film of $\mathrm{La}_{0}{ }_{7} \mathrm{Sr} 0 . \mathrm{MnO}_{3} / \mathrm{SrTiO}_{3}$ : ac magnetic susceptibility and magnetic viscosity investigations, J. Appl. Phys. 128 (2020) 73903.

2. N. Mottaghi, R. B. Trappen, S. Y. Sarraf, M. S. Seehra, and M. B. Holcomb, "Magnetocaloric investigations show magnetic inhomogeneity in a $7.6 \mathrm{~nm}$ thin film of $\mathrm{La0} .7 \mathrm{Sr} 0.3 \mathrm{MnO} 3 / \mathrm{SrTiO}_{3}$," J. Alloys Compd., p. 154200, 2020.

3. N. Mottaghi et al., "Observation and interpretation of negative remanent magnetization and inverted hysteresis loops in a thin film of La0.7Sr0.3MnO3," J. Phys. Condens. Matter, vol. 30, no. 40, p. 405804, 2018.

4. N. Mottaghi et al., "Insights into the magnetic dead layer in $\mathrm{La}_{0.7} \mathrm{Sr} 0.3 \mathrm{MnO} 3$ thin films from temperature, magnetic field and thickness dependence of their magnetization," AIP Adv., vol. 8, no. 5, p. $056319,2018$.

5. N. Mottaghi et al., "Ag/Pd core-shell nanoparticles by a successive method: Pulsed laser ablation of $\mathrm{Ag}$ in water and reduction reaction of PdCl2,” Appl. Surf. Sci., vol. 292, pp. 892-897, 2014. 
6. S. Kumari, N. Mottaghi, C.-Y. Huang, R. Trappen, G. Bhandari, S. Yousefi, G. Cabrera, M.S. Seehra, M.B. Holcomb, Effects of Oxygen Modification on the Structural and Magnetic Properties of Highly Epitaxial La0.7Sr0.3MnO3 (LSMO) thin films, Sci. Rep. 10 (2020) 3659. https://doi.org/10.1038/s41598020-60343-5.

7. Trappen, Robbyn, Alexander J. Grutter, Chih-Yeh Huang, Aubrey Penn, Navid Mottaghi, Saeed Yousefi, Allison Haertter, Shalini Kumari, James LeBeau, Brian J. Kirby, and Mikel B. Holcomb. (2019) Effect of oxygen stoichiometry on the magnetization profiles and negative magnetization in LSMO thin films, Journal of Applied Physics. American Institute of Physics, 126(10), p. 105301. https://aip.scitation.org/doi/abs/10.1063/1.5111858

8. Penn, A. N., Trappen, R., Mottaghi, N., Huang, C.-Y., Kumar, A., Holcomb, M., \& LeBeau, J. M. (2019). Explaining the Magnetic Properties of Oxygen Deficient LSMO Thin Films by iDPC. Microscopy and Microanalysis, 25(S2), 1748-1749. https://doi.org/DOI: 10.1017/S1431927619009474

9. S.Y. Sarraf, R. Trappen, S. Kumari, G. Bhandari, N. Mottaghi, C.Y. Huang, G.B. Cabrera, A.D. Bristow, and M.B. Holcomb (2019). Application of wavelet analysis on transient reflectivity in ultra-thin films. Optics Express, 27(10), 14684-14694. https://doi.org/10.1364/OE.27.014684

10. Saeed Yousefi Sarraf, Sobhit Singh, Andrés Camilo Garcia-Castro, Robbyn Trappen, Navid Mottaghi, Guerau B. Cabrera, Chih-Yeh Huang, Shalini Kumari, Ghadendra Bhandari, Alan D. Bristow, Aldo H. Romero, and Mikel B. Holcomb., "Surface Recombination in Ultra-Fast Carrier Dynamics of Perovskite Oxide La0.7Sr0.3MnO3 Thin Films,” ACS Nano,: DOI: 10.1021/acsnano.8b09595.

\subsubsection{Presentations}

1. Navid Mottaghi, Mohindar S. Seehra, Jianhang Shi, Menka Jain, Spin dynamics and relaxation in thin film La0.7Sro.3MnO3/SrTiO3: ac magnetic susceptibility and magnetic viscosity investigations, MMM 2020 Virtual Conference, November 2-6, 2020.

2. Navid Mottaghi, Shalini Kumari, Mohindar S. Seehra, Mikel Holcomb. Time dynamics and magnetic viscosity measurements in inhomogeneous La0.7Sro.3MnO3 films. Annual Conference on Magnetism and Magnetic Materials, November 4-9, 2019 Las Vegas, California.

3. Navid Mottaghi, Robbyn B. Trappen, Saeed Yousefi Sarraf, Mohindar S. Seehra, Mikel B. Holcomb, Magnetocaloric investigations show magnetic inhomogeneity in a $7.6 \mathrm{~nm}$ thin film of

La0.7Sro.3MnO3/SrTiO3, AVS 66th International Symposium \& Exhibition, October 20-25, 2019, Columbus, Ohio.

4. Navid Mottaghi et al., Insights into the Magnetic Dead Layer in La0.7SrO.3MnO3 Thin Films from Temperature, Magnetic Field and Thickness Dependence of their Magnetization, AVS 65th conference, October 21-26, 2018, Long Beach, California.

5. N. Mottaghi et al., Insights into the magnetic dead layer in $\mathrm{La} 0.7 \mathrm{Sr} 0.3 \mathrm{MnO} 3$ thin films from temperature, magnetic field and thickness dependence of their magnetization, 2017 MMM Conference, November 610, 2017 in Pittsburgh, USA.

6. Navid Mottaghi et al., Substrate effects on magnetic properties of LSMO thin films, Bulletin of the American Physical Society, 2018. 


\section{References}

[1] I.P. Muthuselvam, R.N. Bhowmik, Grain size dependent magnetization, electrical resistivity and magnetoresistance in mechanically milled $\mathrm{La}_{0.67} \mathrm{Sr}_{0.33} \mathrm{MnO}_{3}$, J. Alloy. Compd. TA - TT -. 511 (2012) 22-30. https://doi.org/10.1016/j.jallcom.2011.09.046 LK https://libwvu.on.worldcat.org/oclc/5902827706.

[2] R. Peng, H.C. Xu, M. Xia, J.F. Zhao, X. Xie, D.F. Xu, B.P. Xie, D.L. Feng, Tuning the dead-layer behavior of $\mathrm{La}_{0.7} \mathrm{Sr}_{0.3} \mathrm{MnO}_{3} / \mathrm{SrTiO}_{3}$ via interfacial engineering, Appl. Phys. Lett. 104 (2014) 81606.

[3] M. Huijben, L.W. Martin, Y.-H. Chu, M.B. Holcomb, P. Yu, G. Rijnders, D.H.A. Blank, R. Ramesh, Critical thickness and orbital ordering in ultrathin $\mathrm{La}_{0.7} \mathrm{Sr}_{0.3} \mathrm{MnO}_{3}$ films, Phys. Rev. B. 78 (2008) 94413. https://doi.org/10.1103/PhysRevB.78.094413.

[4] A.H. Morrish, The Physical Principles of Magnetism, Wiley-IEEE PRESS, n.d. https://doi.org/10.1109/9780470546581.

[5] A.J. Bray, Nature of the Griffiths phase, Phys. Rev. Lett. 59 (1987) 586-589. https://doi.org/10.1103/PhysRevLett.59.586.

[6] S.F. Edwards, P.W. Anderson, Theory of spin glasses, J. Phys. F Met. Phys. 5 (1975) 965-974. https://doi.org/10.1088/0305-4608/5/5/017.

[7] G.H. Jonker, J.H. Van Santen, Ferromagnetic compounds of manganese with perovskite structure, Physica. 16 (1950) 337-349. https://doi.org/https://doi.org/10.1016/0031-8914(50)90033-4.

[8] N. Izyumskaya, Y. Alivov, H. Morkoç, Oxides, oxides, and more oxides: High-oxides, ferroelectrics, ferromagnetics, and multiferroics, Crit. Rev. Solid State Mater. Sci. 34 (2009) 89179. https://doi.org/10.1080/10408430903368401.

[9] H. Boschker, M. Huijben, A. Vailionis, J. Verbeeck, S. van Aert, M. Luysberg, S. Bals, G. van Tendeloo, E.P. Houwman, G. Koster, D.H.A. Blank, G. Rijnders, Optimized fabrication of highquality $\mathrm{La}_{0.67} \mathrm{Sr}_{0.33} \mathrm{MnO}_{3}$ thin films considering all essential characteristics, J. Phys. D. Appl. Phys. 44 (2011) 205001. https://doi.org/10.1088/0022-3727/44/20/205001.

[10] A. Vailionis, H. Boschker, W. Siemons, E.P. Houwman, D.H.A. Blank, G. Rijnders, G. Koster, Misfit strain accommodation in epitaxial $\mathrm{ABO} 3$ perovskites: Lattice rotations and lattice modulations, Phys. Rev. B Condens. Matter Mater. Phys. 83 (2011). https://doi.org/10.1103/PhysRevB.83.064101.

[11] C. Zener, Interaction Between the $d$ Shells in the Transition Metals, Phys. Rev. 81 (1951) 440-444. https://doi.org/10.1103/PhysRev.81.440. 
[12] J.B. Goodenough, Theory of the Role of Covalence in the Perovskite-Type Manganites [La, M(II)] $\mathrm{MnO}_{3}$, Phys. Rev. 100 (1955) 564-573. https://doi.org/10.1103/PhysRev.100.564.

[13] P.W. Anderson, Antiferromagnetism. Theory of Superexchange Interaction, Phys. Rev. 79 (1950) 350-356. https://doi.org/10.1103/PhysRev.79.350.

[14] C. Zener, Interaction between the \$d\$-Shells in the Transition Metals. II. Ferromagnetic Compounds of Manganese with Perovskite Structure, Phys. Rev. 82 (1951) 403-405. https://doi.org/10.1103/PhysRev.82.403.

[15] J.H. Van Santen, G.H. Jonker, Electrical conductivity of ferromagnetic compounds of manganese with perovskite structure, Physica. 16 (1950) 599-600. https://doi.org/https://doi.org/10.1016/00318914(50)90104-2.

[16] J. Huang, H. Wang, X. Sun, X. Zhang, H. Wang, Multifunctional $\mathrm{La}_{0.67} \mathrm{Sr}_{0.33} \mathrm{MnO}_{3}$ (LSMO) Thin Films Integrated on Mica Substrates toward Flexible Spintronics and Electronics, ACS Appl. Mater. Interfaces. 10 (2018) 42698-42705. https://doi.org/10.1021/acsami.8b16626.

[17] V. Borisov, S. Ostanin, I. Mertig, Multiferroic properties of the $\mathrm{PbTiO}_{3} / \mathrm{La}_{2 / 3} \mathrm{Sr}_{1 / 3} \mathrm{MnO}_{3}$ interface studied from first principles, J. Phys. Condens. Matter. 29 (2017) 175801. https://doi.org/10.1088/1361-648X/aa6318.

[18] Y.H. Ren, M. Ebrahim, H.B. Zhao, G. Lüpke, Z.A. Xu, V. Adyam, Q. Li, Time-resolved optical studies of spin and quasiparticle dynamics in colossal magnetoresistance materials: $\mathrm{La}_{0.67} \mathrm{Ca}_{0.33} \mathrm{MnO}_{3}$ and $\mathrm{Sr}_{2} \mathrm{FeMoO}_{6}$, Phys. Rev. B. $78 \quad$ (2008) 14408 . https://doi.org/10.1103/PhysRevB.78.014408.

[19] C. Moreno, C. Munuera, S. Valencia, F. Kronast, X. Obradors, C. Ocal, Reversible resistive switching and multilevel recording in $\mathrm{La} 0.7 \mathrm{Sr} 0.3 \mathrm{MnO} 3$ thin films for low cost nonvolatile memories, Nano Lett. 10 (2010) 3828-3835.

[20] L. You, C. Lu, P. Yang, G. Han, T. Wu, U. Luders, W. Prellier, K. Yao, L. Chen, J. Wang, Uniaxial magnetic anisotropy in $\mathrm{La}_{0.7} \mathrm{Sr}_{0.3} \mathrm{MnO}_{3}$ thin films induced by multiferroic $\mathrm{BiFeO}_{3}$ with striped ferroelectric domains, Adv. Mater. 22 (2010) 4964-4968.

[21] R. Martínez, A. Kumar, R. Palai, J.F. Scott, R.S. Katiyar, Impedance spectroscopy analysis of Ba. ${ }_{7} \mathrm{Sr}_{0.3} \mathrm{TiO}_{3} / \mathrm{La}_{0.7} \mathrm{Sr}_{0 .}{ }_{3} \mathrm{MnO}_{3}$ heterostructure, J. Phys. D. Appl. Phys. 44 (2011) 105302.

[22] J. Curiale, M. Granada, H.E. Troiani, R.D. Sánchez, A.G. Leyva, P. Levy, K. Samwer, Magnetic dead layer in ferromagnetic manganite nanoparticles, Appl. Phys. Lett. 95 (2009) 43106. https://doi.org/10.1063/1.3187538. 
[23] Å. Monsen, J.E. Boschker, F. Macià, J.W. Wells, P. Nordblad, A.D. Kent, R. Mathieu, T. Tybell, E. Wahlström, Thickness dependence of dynamic and static magnetic properties of pulsed laser deposited $\mathrm{La}_{0.7} \mathrm{Sr}_{0.3} \mathrm{MnO}_{3}$ films on $\mathrm{SrTiO}_{3}(001)$, J. Magn. Magn. Mater. 369 (2014) 197-204. https://doi.org/http://dx.doi.org/10.1016/j.jmmm.2014.06.038.

[24] N. Mottaghi, M.S. Seehra, R. Trappen, S. Kumari, C.Y. Huang, S. Yousefi, G.B. Cabrera, A.H. Romero, M.B. Holcomb, Insights into the magnetic dead layer in $\mathrm{La}_{0.7} \mathrm{Sr}_{0.3} \mathrm{MnO}_{3}$ thin films from temperature, magnetic field and thickness dependence of their magnetization, AIP Adv. 8 (2018) 56319. https://doi.org/10.1063/1.5005913.

[25] S. Thota, J.H. Shim, M.S. Seehra, Size-dependent shifts of the Néel temperature and optical bandgap in NiO nanoparticles, J. Appl. Phys. 114 (2013) 214307. https://doi.org/10.1063/1.4838915.

[26] O. Iglesias, A. Labarta, Finite-size and surface effects in maghemite nanoparticles: Monte Carlo simulations, Phys. Rev. B. 63 (2001) 184416. https://doi.org/10.1103/PhysRevB.63.184416.

[27] S. Roy, I. Dubenko, D.D. Edorh, N. Ali, S. Mno, Size induced variations in structural and magnetic properties of double, J. Appl. Phys. 96 (2004) 1202-1208. https://doi.org/10.1063/1.1760230.

[28] X. Xiao, X. Zhao, J. Guo, W. Liu, Z. Zhang, Cluster spin-glass behavior in Ni2In-type Mn-Cu-Ga alloys, J. Alloys Compd. 816 (2020) 152678.

[29] E. V Sampathkumaran, A. Niazi, Superparamagnetic-like ac susceptibility behavior in the partially disordered antiferromagnetic compound $\mathrm{Ca}_{3} \mathrm{CoRhO}_{6}$, Phys. Rev. B. 65 (2002) 180401. https://doi.org/10.1103/PhysRevB.65.180401.

[30] E. Snider, N. Dasenbrock-Gammon, R. McBride, M. Debessai, H. Vindana, K. Vencatasamy, K. V Lawler, A. Salamat, R.P. Dias, Room-temperature superconductivity in a carbonaceous sulfur hydride, Nature. 586 (2020) 373-377. https://doi.org/10.1038/s41586-020-2801-z.

[31] J. Chang, Y.-S. Park, S.-K. Kim, Atomically flat single-terminated $\mathrm{SrTiO}_{3}$ (111) surface, Appl. Phys. Lett. 92 (2008) 152910.

[32] L.Q. Jiang, J.K. Guo, H.B. Liu, M. Zhu, X. Zhou, P. Wu, C.H. Li, Prediction of lattice constant in cubic perovskites, J. Phys. Chem. Solids. 67 (2006) 1531-1536. https://doi.org/https://doi.org/10.1016/j.jpcs.2006.02.004.

[33] G. Koster, B.L. Kropman, G.J.H.M. Rijnders, D.H.A. Blank, H. Rogalla, Quasi-ideal strontium titanate crystal surfaces through formation of strontium hydroxide, Appl. Phys. Lett. 73 (1998) 2920-2922.

[34] I. Velasco-Davalos, R. Thomas, A. Ruediger, Realization of single-termination $\mathrm{SrTiO}_{3}$ (100) 
surfaces by a microwave-induced hydrothermal process, Appl. Phys. Lett. 103 (2013) 202905. https://doi.org/10.1063/1.4831681.

[35] D. Pesquera, G. Herranz, A. Barla, E. Pellegrin, F. Bondino, E. Magnano, F. Sánchez, J. Fontcuberta, Surface symmetry-breaking and strain effects on orbital occupancy in transition metal perovskite epitaxial films, Nat. Commun. 3 (2012) 1189. https://doi.org/10.1038/ncomms2189.

[36] G. Koster, G. Rijnders, D.H.A. Blank, H. Rogalla, Surface morphology determined by (0 001$)$ singlecrystal SrTiO3termination, Phys. C Supercond. Its Appl. 339 (2000) 215-230. https://doi.org/10.1016/S0921-4534(00)00363-4.

[37] K. Szot, W. Speier, Surfaces of reduced and oxidized $\mathrm{SrTiO}_{3}$ from atomic force microscopy, Phys. Rev. B. 60 (1999) 5909-5926. https://doi.org/10.1103/PhysRevB.60.5909.

[38] T. Ohnishi, K. Shibuya, M. Lippmaa, D. Kobayashi, H. Kumigashira, M. Oshima, H. Koinuma, Preparation of thermally stable $\mathrm{TiO}_{2}$-terminated SrTiO3 (100) substrate surfaces, Appl. Phys. Lett. 85 (2004) 272-274. https://doi.org/10.1063/1.1771461.

[39] M. Kawasaki, K. Takahashi, T. Maeda, R. Tsuchiya, M. Shinohara, O. Ishiyama, T. Yonezawa, M. Yoshimoto, H. Koinuma, Atomic Control of the $\mathrm{SrTiO}_{3}$ Crystal Surface, Science (80-. ). 266 (1994) 1540 LP - 1542. https://doi.org/10.1126/science.266.5190.1540.

[40] V. Leca, G. Rijnders, G. Koster, D.H.A. Blank, H. Rogalla, Wet Etching Methods for Perovskite Substrates, MRS Proc. 587 (1999) O3.6. https://doi.org/DOI: 10.1557/PROC-587-O3.6.

[41] R. Bachelet, F. Sánchez, J. Santiso, C. Munuera, C. Ocal, J. Fontcuberta, Self-assembly of SrTiO3 (001) chemical-terminations: a route for oxide-nanostructure fabrication by selective growth, Chem. Mater. 21 (2009) 2494-2498.

[42] J. Klein, C. Höfener, L. Alff, R. Gross, Laser ablation of manganite thin films monitored by in situ RHEED, J. Magn. Magn. Mater. 211 (2000) 9-15.

[43] J. Li, W. Peng, K. Chen, P. Wang, H. Chu, Y. Chen, D. Zheng, Growth and in situ high-pressure reflection high energy electron diffraction monitoring of oxide thin films, Sci. China Physics, Mech. Astron. 56 (2013) 2312-2326. https://doi.org/10.1007/s11433-013-5352-6.

[44] D.H.A. Blank, G.J.H.M. Rijnders, G. Koster, H. Rogalla, In-situ monitoring by reflective high energy electron diffraction during pulsed laser deposition, Appl. Surf. Sci. 138-139 (1999) 17-23. https://doi.org/https://doi.org/10.1016/S0169-4332(98)00470-X.

[45] M. Yasaka, X-ray thin-film measurement techniques, Rigaku J. 26 (2010) 1-9. 
[46] L. Belliard, A. Huynh, B. Perrin, A. Michel, G. Abadias, C. Jaouen, Elastic properties and phonon generation in Mo/Si superlattices, Phys. Rev. B - Condens. Matter Mater. Phys. 80 (2009). https://doi.org/10.1103/PhysRevB.80.155424.

[47] S. Singh, S. Basu, P. Bhatt, A.K. Poswal, Kinetics of alloy formation at the interfaces in a Ni-Ti multilayer: X-ray and neutron reflectometry study, Phys. Rev. B. 79 (2009) 195435. https://link.aps.org/doi/10.1103/PhysRevB.79.195435.

[48] J.-S. Lee, D.A. Arena, P. Yu, C.S. Nelson, R. Fan, C.J. Kinane, S. Langridge, M.D. Rossell, R. Ramesh, C.-C. Kao, Hidden Magnetic Configuration in Epitaxial La1-xSrxMnO3 Films, Phys. Rev. Lett. 105 (2010) 257204. https://doi.org/10.1103/PhysRevLett.105.257204.

[49] Š́ Málková, F. Long, R. V Stahelin, S. V Pingali, D. Murray, W. Cho, M.L. Schlossman, X-Ray Reflectivity Studies of cPLA2 $\alpha-C 2$ Domains Adsorbed onto Langmuir Monolayers of SOPC, Biophys. J. 89 (2005) 1861-1873. https://doi.org/https://doi.org/10.1529/biophysj.105.061515.

[50] Y.T. Chen, H.S. Su, C.H. Hung, P.W. Yang, Y. Hu, T.L. Lin, M.T. Lee, U.S. Jeng, X-ray Reflectivity Studies on the Mixed Langmuir-Blodgett Monolayers of Thiol-Capped Gold Nanoparticles, Dipalmitoylphosphatidylcholine, and Sodium Dodecyl Sulfate, Langmuir. 33 (2017) 10886-10897. https://doi.org/10.1021/acs.langmuir.7b01559.

[51] C. Kittel, Introduction to solid state physics, Wiley, 2005.

[52] P. Weiss, L’hypothèse du champ moléculaire et la propriété ferromagnétique, (1907).

[53] W. Heisenberg, Mehrkörperproblem und Resonanz in der Quantenmechanik, Zeitschrift Für Phys. 38 (1926) 411-426. https://doi.org/10.1007/BF01397160.

[54] S. Blundell, Magnetism in condensed matter, (2003).

[55] K. Charles, Introduction to Solid State Physics, Wiley, 1986. http://www.ncbi.nlm.nih.gov/pubmed/22118571.

[56] C.P. Bean, J.D. Livingston, Superparamagnetism, J. Appl. Phys. 30 (1959) S120-S129. https://doi.org/10.1063/1.2185850.

[57] L. Néel, Some theoretical aspects of rock-magnetism, Adv. Phys. 4 (1955) 191-243. https://doi.org/10.1080/00018735500101204.

[58] D.L. Leslie-Pelecky, R.D. Rieke, Magnetic Properties of Nanostructured Materials, Chem. Mater. 8 (1996) 1770-1783. https://doi.org/10.1021/cm960077f.

[59] W.F. Brown, Thermal Fluctuations of a Single-Domain Particle, Phys. Rev. 130 (1963) 1677-1686. 
https://doi.org/10.1103/PhysRev.130.1677.

[60] A. Aharoni, Effect of a Magnetic Field on the Superparamagnetic Relaxation Time, Phys. Rev. 177 (1969) 793-796. https://doi.org/10.1103/PhysRev.177.793.

[61] R.W. Chantrell, Magnetic viscosity of recording media, J. Magn. Magn. Mater. 95 (1991) 365-378. https://doi.org/https://doi.org/10.1016/0304-8853(91)90231-X.

[62] L. Néel, Influence des fluctuations thermiques sur l'aimantation de grains ferromagnétiques très fins, Comptes Rendus Hebd. Des Seances L Acad. Des Sci. 228 (1949) 664-666.

[63] S. Shtrikman, E.P. Wohlfarth, The theory of the Vogel-Fulcher law of spin glasses, Phys. Lett. A. 85 (1981) 467-470. https://doi.org/https://doi.org/10.1016/0375-9601(81)90441-2.

[64] S. Shtrikman, E.P. Wohlfarth, The theory of the Vogel-Fulcher law of spin glasses, Phys. Lett. A. 85 (1981) 467-470. https://doi.org/https://doi.org/10.1016/0375-9601(81)90441-2.

[65] F.C. Fonseca, G.F. Goya, R.F. Jardim, R. Muccillo, N.L.V. Carreño, E. Longo, E.R. Leite, Superparamagnetism and magnetic properties of Ni nanoparticles embedded in SiO2, Phys. Rev. B - Condens. Matter Mater. Phys. $66 \quad$ (2002) 1044061-1044065. https://doi.org/10.1103/PhysRevB.66.104406.

[66] A. Ettayfi, R. Moubah, E.K. Hlil, S. Colis, M. Lenertz, A. Dinia, H. Lassri, Spin wave and percolation studies in epitaxial $\mathrm{La}_{2 / 3} \mathrm{Sr}_{1 / 3} \mathrm{MnO}_{3}$ thin films grown by pulsed laser deposition, J. Magn. Magn. Mater. 409 (2016) 34-38.

[67] S. Roy, I. Dubenko, D.D. Edorh, N. Ali, Size induced variations in structural and magnetic properties of double exchange La0.8Sr0.2MnO3- $\delta$ nano-ferromagnet, J. Appl. Phys. 96 (2004) 1202-1208. https://doi.org/10.1063/1.1760230.

[68] W. Kipferl, M. Dumm, P. Kotissek, F. Steinbauer, G. Bayreuther, Bloch's law for epitaxial ultrathin dot arrays with uniaxial magnetic anisotropy, J. Appl. Phys. 95 (2004) 7417-7419. https://doi.org/10.1063/1.1676055.

[69] N. Mottaghi, R.B. Trappen, S. Kumari, C.Y. Huang, S. Yousefi, G.B. Cabrera, M. Aziziha, A. Haertter, M.B. Johnson, M.S. Seehra, M.B. Holcomb, Observation and interpretation of negative remanent magnetization and inverted hysteresis loops in a thin film of $\mathrm{La}_{0.7} \mathrm{Sr}_{0.3} \mathrm{MnO}_{3}$, J. Phys. Condens. Matter. 30 (2018) 405804. https://doi.org/10.1088/1361-648X/aade14.

[70] T. Maity, D. Kepaptsoglou, M. Schmidt, Q. Ramasse, S. Roy, Observation of complete inversion of the hysteresis loop in a bimodal magnetic thin film, Phys. Rev. B. 95 (2017) 100401. https://link.aps.org/doi/10.1103/PhysRevB.95.100401. 
[71] S. Gu, W. He, M. Zhang, T. Zhuang, Y. Jin, H. ElBidweihy, Y. Mao, J.H. Dickerson, M.J. Wagner, E. Della Torre, L.H. Bennett, Physical Justification for Negative Remanent Magnetization in Homogeneous Nanoparticles, Sci. Rep. 4 (2014) 6267. http://dx.doi.org/10.1038/srep06267.

[72] J. Yang, J. Kim, J. Lee, S. Woo, J. Kwak, J. Hong, M. Jung, Inverted hysteresis loops observed in a randomly distributed cobalt nanoparticle system, Phys. Rev. B. 78 (2008) 94415. https://link.aps.org/doi/10.1103/PhysRevB.78.094415.

[73] X. Yan, Y. Xu, Negative remanence in magnetic nanostructures, J. Appl. Phys. 79 (1996) 6013. https://doi.org/10.1063/1.362137.

[74] S.B. Porter, M. Venkatesan, P. Dunne, B. Doudin, K. Rode, J.M.D. Coey, Magnetic Dead Layers in $\mathrm{La}_{0.7} \mathrm{Sr}_{0.3} \mathrm{MnO}_{3} \quad$ Revisited, IEEE Trans. Magn. $53 \quad$ (2017) 1-4. https://doi.org/10.1109/TMAG.2017.2731124.

[75] E.P. Wohlfarth, The coefficient of magnetic viscosity, J. Phys. F Met. Phys. 14 (1984) L155-L159. https://doi.org/10.1088/0305-4608/14/8/005.

[76] N. Mottaghi, M.S. Seehra, J. Shi, M. Jain, M.B. Holcomb, Spin dynamics and relaxation in $7.6 \mathrm{~nm}$ thin film of $\mathrm{La} 0.7 \mathrm{Sr} 0.3 \mathrm{MnO} 3 / \mathrm{SrTiO} 3$ : ac magnetic susceptibility and magnetic viscosity investigations, J. Appl. Phys. 128 (2020) 073903. https://doi.org/10.1063/5.0017765.

[77] M.M. Ibrahim, S. Darwish, M.S. Seehra, Nonlinear temperature variation of magnetic viscosity in nanoscale $\mathrm{FeOOH}$ particles, Phys. Rev. B. $51 \quad$ (1995) 2955-2959. https://link.aps.org/doi/10.1103/PhysRevB.51.2955.

[78] J. Mira, J. Rivas, F. Rivadulla, C. Vázquez-Vázquez, M.A. López-Quintela, Change from first- to second-order magnetic phase transition in $\mathrm{La}_{2 / 3}(\mathrm{Ca}, \mathrm{Sr})_{1 / 3} \mathrm{MnO}_{3}$ perovskites, Phys. Rev. B. 60 (1999) 2998-3001. https://link.aps.org/doi/10.1103/PhysRevB.60.2998.

[79] L.D. Landau, On the theory of phase transitions, Ukr. J. Phys. 11 (1937) 19-32.

[80] N. Mottaghi, R.B. Trappen, S.Y. Sarraf, M.S. Seehra, M.B. Holcomb, Magnetocaloric investigations show magnetic inhomogeneity in a $7.6 \mathrm{~nm}$ thin film of $\mathrm{La}_{0.7} \mathrm{Sr}_{0.3} \mathrm{MnO}_{3} / \mathrm{SrTiO}_{3}$, J. Alloys Compd. (2020) 154200. https://doi.org/https://doi.org/10.1016/j.jallcom.2020.154200.

[81] A. Rostamnejadi, M. Venkatesan, P. Kameli, H. Salamati, J.M.D. Coey, Magnetocaloric effect in $\mathrm{La}_{0.67} \mathrm{Sr}_{0.33} \mathrm{MnO}_{3}$ manganite above room temperature, J. Magn. Magn. Mater. 323 (2011) 22142218. https://doi.org/10.1016/j.jmmm.2011.03.036.

[82] V.S. Kumar, R. Chukka, Z. Chen, P. Yang, L. Chen, Strain dependent magnetocaloric effect in La0.67Sr0.33MnO3 thin-films, AIP Adv. 3 (2013) 52127. https://doi.org/10.1063/1.4807739. 
[83] A. Ettayfi, R. Moubah, A. Boutahar, E.K. Hlil, H. Lassri, Structural, Magnetic, Magnetocaloric, and Critical Exponent Properties of La0.67Sr0.33MnO3 Powders Synthesized by Solid-State Reaction, J. Supercond. Nov. Magn. 29 (2016) 133-138. https://doi.org/10.1007/s10948-015-3212-5.

[84] X. Zhang, H. Zhang, M. Qian, L. Geng, Enhanced magnetocaloric effect in Ni-Mn-Sn-Co alloys with two successive magnetostructural transformations, Sci. Rep. 8 (2018) 8235. https://doi.org/10.1038/s41598-018-26564-5.

[85] R. M'nassri, M.M. Nofal, P. de Rango, N. Chniba-Boudjada, Magnetic entropy table-like shape and enhancement of refrigerant capacity in La1.4Ca1.6Mn2O7-La1.3Eu0.1Ca1.6Mn2O7 composite, RSC Adv. 9 (2019) 14916-14927. https://doi.org/10.1039/C9RA00984A.

[86] T.L. Phan, N. Tran, D.H. Kim, N.T. Dang, D.H. Manh, T.N. Bach, C.L. Liu, B.W. Lee, Magnetic and Magnetocaloric Properties of $\mathrm{Zn}^{\wedge} \mathrm{sub} 1-\mathrm{x}^{\wedge} \mathrm{Co}^{\wedge} \mathrm{sub} \mathrm{x}^{\wedge} \mathrm{Fe}^{\wedge} \mathrm{sub} 2^{\wedge} \mathrm{O}^{\wedge} \mathrm{sub} 4^{\wedge}$ Nanoparticles, J. Electron. Mater. 46 (2017) 4214-4226. https://doi.org/http://dx.doi.org/10.1007/s11664-017-53592.

[87] N. Amama, H. Omrani, W. Cheikhrouhou Koubaa, M. Koubaa, A. Cheikhrouhou, Magnetocaloric Effect and Critical Behavior Investigations in $\mathrm{La}_{0.45} \operatorname{Pr}_{0.2} \mathrm{Sr}_{0.35} \mathrm{MnO}_{3}$ Manganite Oxide, J. Supercond. Nov. Magn. TA - TT -. 32 (2019) 1353-1365. https://doi.org/10.1007/s10948-018-4836-z LK https://libwvu.on.worldcat.org/oclc/7814858703.

[88] J.Y. Law, V. Franco, L.M. Moreno-Ramírez, A. Conde, D.Y. Karpenkov, I. Radulov, K.P. Skokov, O. Gutfleisch, A quantitative criterion for determining the order of magnetic phase transitions using the magnetocaloric effect, Nat. Commun. 9 (2018) 2680. https://doi.org/10.1038/s41467-01805111-w.

[89] M. Pękała, Magnetic field dependence of magnetic entropy change in nanocrystalline and polycrystalline manganites La1-xMxMnO3 (M=Ca,Sr), J. Appl. Phys. 108 (2010) 113913. https://doi.org/10.1063/1.3517831.

[90] H. Oesterreicher, F.T. Parker, Magnetic cooling near Curie temperatures above 300 K, J. Appl. Phys. 55 (1984) 4334-4338. https://doi.org/10.1063/1.333046.

[91] B.R. Dahal, K. Schroeder, M.M. Allyn, R.J. Tackett, Y. Huh, P. Kharel, Near-room-temperature magnetocaloric properties of $\mathrm{La} 1-\mathrm{xSrxMnO} 3(\mathrm{x}=0.11,0.17$, and 0.19$)$ nanoparticles, Mater. Res. Express. 5 (2018) 106103. https://doi.org/10.1088/2053-1591/aadabd.

[92] A. Ettayfi, R. Moubah, A. Boutahar, E.K. Hlil, H. Lassri, Structural, Magnetic, Magnetocaloric, and Critical Exponent Properties of La0.67Sr0.33MnO3 Powders Synthesized by Solid-State Reaction, 
J. Supercond. Nov. Magn. 29 (2016) 133-138. https://doi.org/10.1007/s10948-015-3212-5.

[93] C. V Topping, S.J. Blundell, AC susceptibility as a probe of low-frequency magnetic dynamics, J. Phys. Condens. Matter. 31 (2018) 13001.

[94] K.S. Cole, Cole. R. H 1941, Dispersion and Absorption in Dielectrics, J. Chem. Phys. 9 (n.d.) 341351. https://doi.org/https://doi.org/10.1063/1.1750906.

[95] H. Nishihara, K. Komiyama, I. Oguro, T. Kanomata, V. Chernenko, Magnetization processes near the Curie temperatures of the itinerant ferromagnets, $\mathrm{Ni}_{2} \mathrm{MnGa}$ and pure nickel, J. Alloys Compd. 442 (2007) 191-193. https://doi.org/10.1016/j.jallcom.2006.09.154.

[96] P.T. Phong, D.H. Manh, L.H. Nguyen, D.K. Tung, N.X. Phuc, I.-J. Lee, Studies of superspin glass state and AC-losses in $\mathrm{La}_{0.7} \mathrm{Sr}_{0.3}$ nanoparticles obtained by high-energy ball-milling, J. Magn. Magn. Mater. 368 (2014) 240-245. https://doi.org/http://dx.doi.org/10.1016/j.jmmm.2014.05.025.

[97] A. Rostamnejadi, H. Salamati, P. Kameli, H. Ahmadvand, Superparamagnetic behavior of La0.67Sr0.33MnO3 nanoparticles prepared via sol-gel method, J. Magn. Magn. Mater. 321 (2009) 3126-3131. https://doi.org/https://doi.org/10.1016/j.jmmm.2009.05.035. 